Old Dominion University

ODU Digital Commons

Civil \& Environmental Engineering Theses \&

Dissertations

Civil \& Environmental Engineering

Spring 2020

\title{
A Rule Based Control Algorithm for on-Ramp Merge With Connected and Automated Vehicles
}

\author{
Ehsan Beheshtitabar \\ Old Dominion University, ehsanbtabar@gmail.com
}

Follow this and additional works at: https://digitalcommons.odu.edu/cee_etds

Part of the Civil Engineering Commons, Transportation Commons, and the Transportation Engineering Commons

\section{Recommended Citation}

Beheshtitabar, Ehsan. "A Rule Based Control Algorithm for on-Ramp Merge With Connected and Automated Vehicles" (2020). Doctor of Philosophy (PhD), Dissertation, Civil \& Environmental Engineering, Old Dominion University, DOI: 10.25777/ttnp-hn26

https://digitalcommons.odu.edu/cee_etds/111

This Dissertation is brought to you for free and open access by the Civil \& Environmental Engineering at ODU Digital Commons. It has been accepted for inclusion in Civil \& Environmental Engineering Theses \& Dissertations by an authorized administrator of ODU Digital Commons. For more information, please contact digitalcommons@odu.edu. 


\title{
A RULE BASED CONTROL ALGORITHM FOR ON-RAMP MERGE WITH CONNECTED AND AUTOMATED VEHICLES
}

\author{
by \\ Ehsan Beheshtitabar \\ B.S. December 2010, Yazd University \\ M.Sc. July 2012, University of Applied Science Technikum Vienna \\ M.Sc. December 2013, Linkoping University
}

\begin{abstract}
A Dissertation Submitted to the Graduate Faculty
of Old Dominion University in partial fulfillment

of the requirements for the degree of
\end{abstract}

DOCTOR OF PHILOSOPHY

CIVIL AND ENVIRONMENTAL ENGINEERING

OLD DOMINION UNIVERSITY

MAY 2020

Approved by:

Prof. Mecit Cetin

Prof. Sherif Ishak

Dr. Hong Yan 


\begin{abstract}
One of the designs for future highways with the flow of Connected Automated Vehicles (CAVs) cars will be a dedicated lane for the CAVs to form platoons and travel with higher speeds and lower headways. The connectivity will enable the formation of platoons of CAVs traveling beside non-platoon lanes. The advent of connectivity between vehicles and the infrastructure will enable advanced control strategies-improving the performance of the traffic-to be incorporated in the traffic system. The merge area in a multilane highway with CAVs is one of the sections which can be enhanced by the operation of a control system.

In this research, a model is developed for investigating the effects of a Rule Based control strategy yielding a more efficient and systematic method for the vehicles joining the highway mainlines comprised of platoon and non-platoon lanes. The actions tested for assisting the merge process included deceleration in the mainlines and lane change to join a platoon in the platoon lane. The model directs every CAV entering a multi-lane highway from an on-ramp, to the rightmost lane of the highway based on the appropriate action which is selected according to the traffic demand conditions and location of the onramp vehicle. To account for car following behavior, the vehicles in the platoon lanes are assumed to have a simplified CACC (cooperative adaptive cruise control) and those in the non-platoon lanes the IDM+ car-following model. The IDM+ car following model is modified with additional controls to incorporate the current technologies of Advanced Driver Assistant Systems (ADAS).

The results of this study showed that the proposed car following model can increase the throughput of the non-platoon lane from approximately 2000 vehicle per hour (vph) to $3400 \mathrm{vph}$ while the platoon lanes each had an average throughput of $3500 \mathrm{vph}$. The merge model enabled higher merging throughput for the merge area compared to current day conditions and displayed the potential for improved traffic performance in a connected environment comprised of platoon and non-platoon lanes. The results of this research will help in the design and development of advanced systems for controlling on-ramp merge sections in the future with CAVs.
\end{abstract}


Copyright, 2020, by Ehsan Beheshtitabar, All Rights Reserved. 


\section{ACKNOWLEDGMENTS}

I would like to express my deepest appreciation to my supervisor, Prof. Mecit Cetin, for his continuous support during my $\mathrm{PhD}$ years and extend my gratitude to my committee members.

I am deeply indebted to my wife and parents for their wholehearted support during this dissertation period. 


\section{Contents}

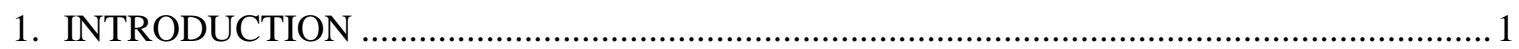

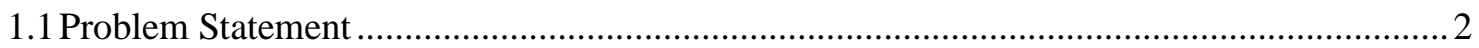

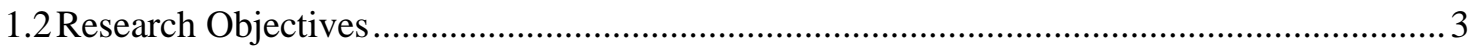

1.3 Scope

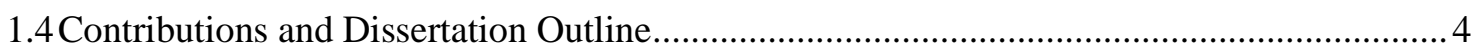

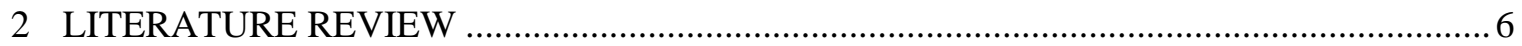

2.1 Connected Automated Vehicles Technology ….................................................................

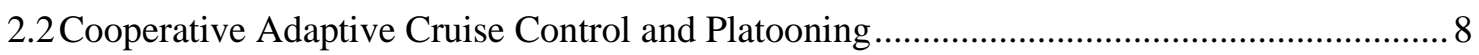

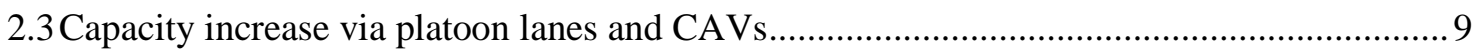

2.4 Automated Merging Control- Current Research.............................................................. 10

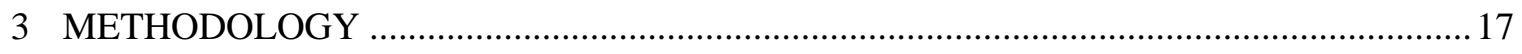

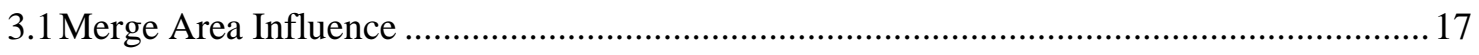

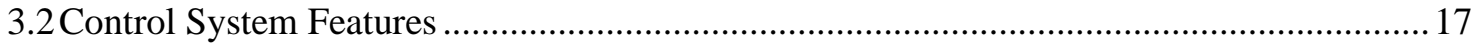

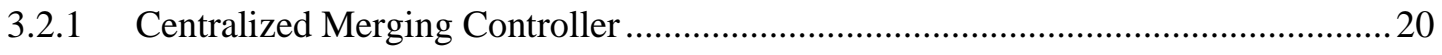

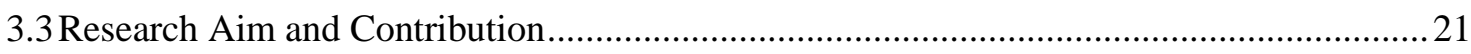

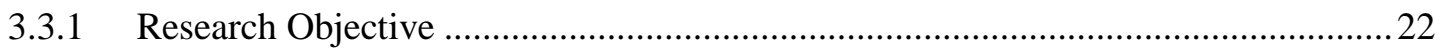

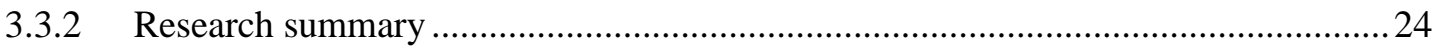

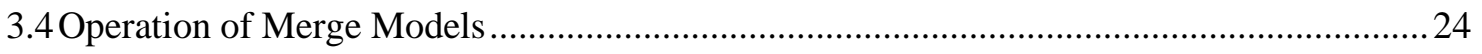

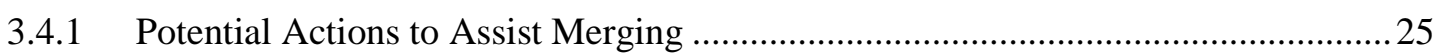

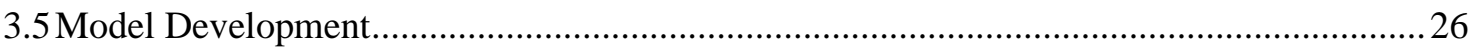




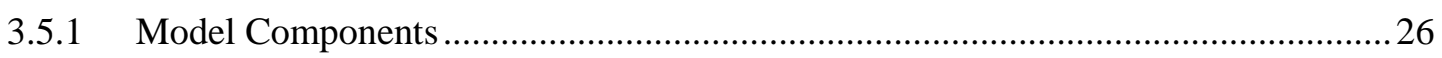

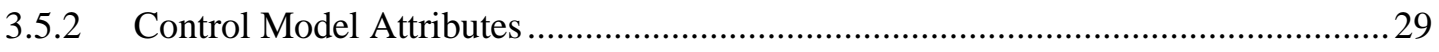

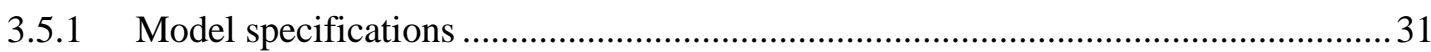

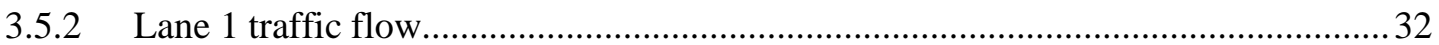

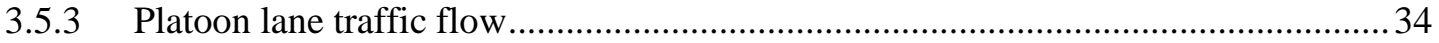

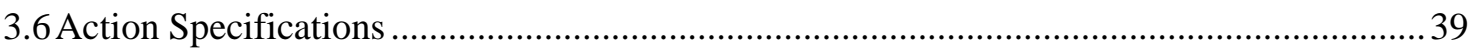

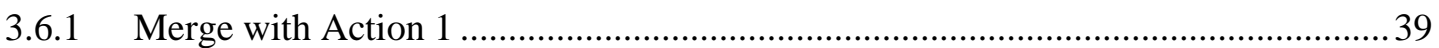

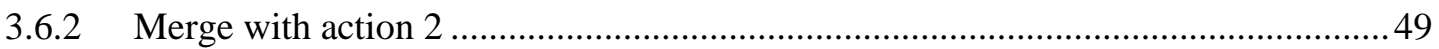

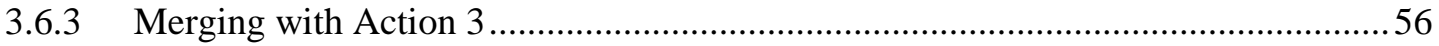

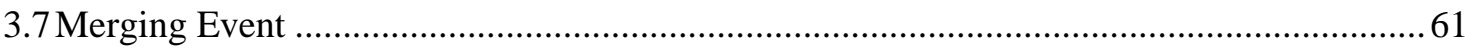

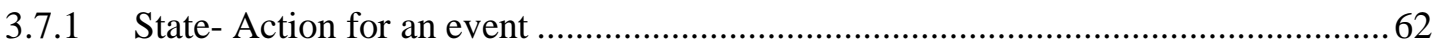

3.7.2 Relation between the merge and traffic factors.......................................................63

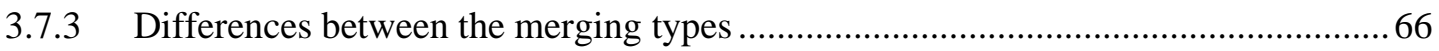

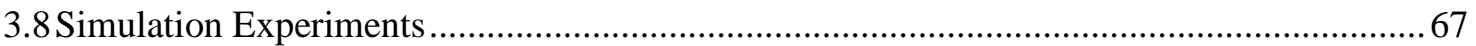

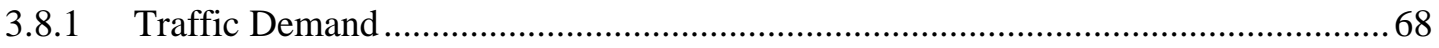

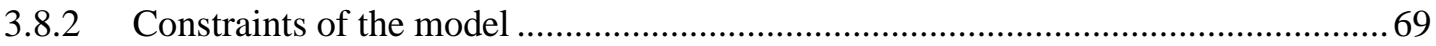

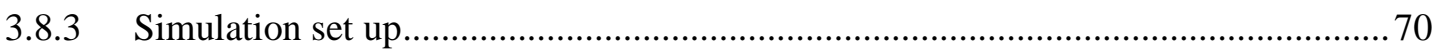

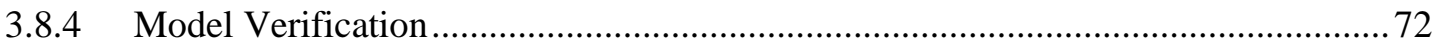

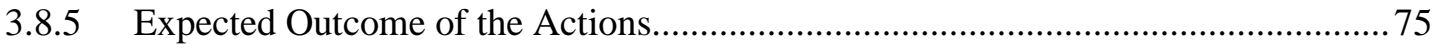

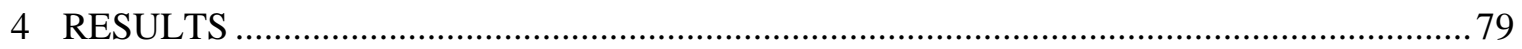

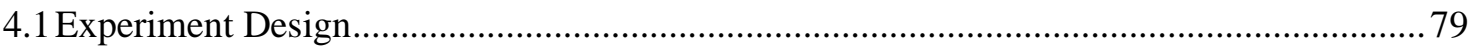




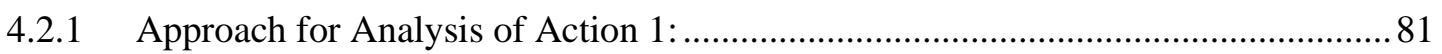

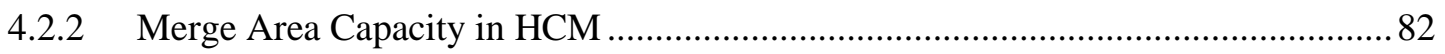

4.2.3 Effect of Geometry on Action Prevention ............................................................. 92

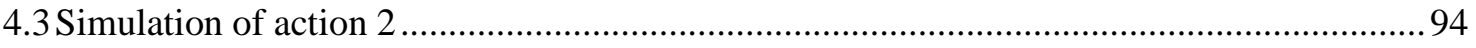

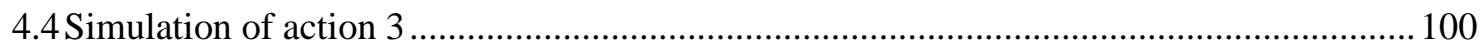

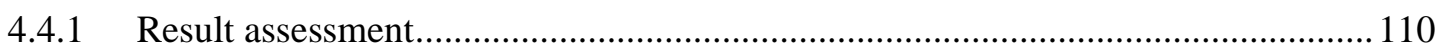

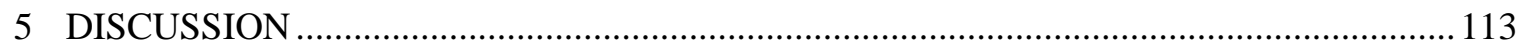

6 CONCLUSION AND FUTURE EXTENSIONS TO THE RESEARCH …..........................116

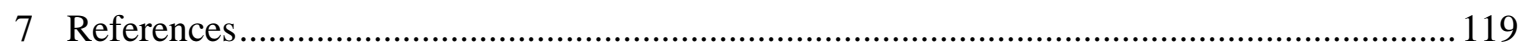

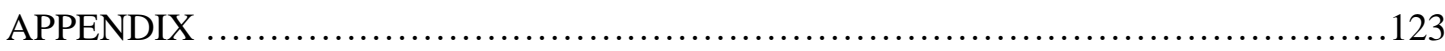

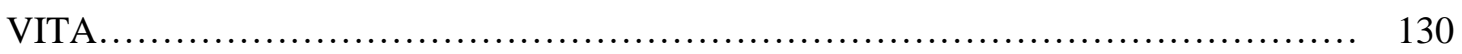




\section{INTRODUCTION}

Connectivity between vehicles is developing to support Intelligent Transport Systems (ITS) in order to solve the safety and congestion problems on highways and intersections. Connected Automated Vehicles (CAVs) will be a commonplace in the traffic in the coming years. Technologies of these vehicles are advancing swiftly, and numerous researches are conducted to study their effects on highways and urban areas. Technologies such as Adaptive Cruise Control (ACC) and Cooperative Adaptive Cruise Control (CACC) besides inter-vehicular connectivity will be utilized by CAVs to enable higher capacities and better traffic performance in urban and rural roads. Many technology estimates predict that within the next few decades, traffic will be dominated by autonomous vehicles. CAVS are being developed not only for providing a more efficient flow of traffic, but also a safer and more reliable means of traveling. The advent of CAVs is expected to alleviate many current traffic problems but numerous challenges from mixed or fully autonomous traffic have yet to be considered.

One of the designs for future highways and freeways which is under investigation for yielding better traffic performance is a separate lane which will be dedicated for the CAVs to form platoons and travel with higher speeds and lower headways than the manual cars, similar to the High Occupancy Vehicle (HOV) lane available at present time (we will call that lane a platoon lane). In CAV environments, platooning represents a viable solution for the capacity problem. The introduction of the platooning concept in traffic was by Varaiya (1993) as a novel form of mobility which can be exploited for maximizing roadway capacity (Varaiya, 1993).

The requirement for the formation of platoons is connectivity between vehicles which can be achieved with the CACC technology. The automation of vehicles will enable further advancement to the connectivity in platoon formation. Additional to the connections between the vehicles, there is connections between the vehicles and the infrastructure, commonly known as V2I (Vehicle to Infrastructure) and I2V (Infrastructure to vehicle). These communications are being designed to be two-way between vehicles and with the infrastructure.

For simplicity, sometimes researchers consider platoons of CAVs as a homogenous entity with a constant intra-platoon headway. The inter-platoon headway can be a varying parameter due to the stochastic and random nature of traffic. It can be expected that in a connected highway with $100 \%$ $\mathrm{CAVs}$, due to the existence of onramps and off-ramps, different destinations in the network etc., the platoons arriving from upstream may not have the same number of vehicles within them. 


\subsection{Problem Statement}

The advent of platoon lanes will impact the traffic operations on a multi-lane highway due to the different driving behavior in different lanes. In the revolutionized highway system, one important problem to tackle is the merging action performance when connectivity between vehicles and platoons on certain lanes are operating. In highway segments with an interrupted flow such as merge influence areas, drivers have been following actions based on their perception and judgment for decades. In today's driving, merging is a demanding task which incorporates a synchronized set of observations, judgments, and actions. This manual merging in many instances yields to unnecessary actions or maneuvers which cause perturbations or disruptions to the mainline flow. Consequently, merging maneuvers are performed differently depending on the driver experience, efficiency, and traffic conditions. This diversity in the merging action from onramp vehicles on highways leads to different observations from merge area capacities. Additionally, merging can initiate speed breakdown if the mainlines are already congested. In the current day traffic, the lack of a suitable gap in the highway for a merging vehicle may force the on-ramp vehicle on the acceleration lane to slow down and seek a larger gap. This slow-downs or decelerations from the mainline and merging vehicles may potentially be inefficient, actions which can be controlled and optimized if the system of the traffic is connected and optimized.

The CAV technology can provide systematic merging of the vehicles such that the traffic performance in the merge influence area is not compromised. In the present-day traffic of highway facilities, drivers utilize the merging gap provided to them which is brought by the randomness of traffic flow. However, transportation would not have the same behavior with CAVs operating. With the need to supply higher traffic demands and reduce traffic delays, the necessity of a controlled merge action within a connected highway environment becomes clear.

With higher demands on mainlines, there would be less suitable gaps for merging vehicles to join the mainline. This would require a merging model which is designed to make appropriate decisions based on relevant traffic parameters. In such a model, onramp vehicles should not be expected to be waiting for gaps, thus implying that a merging model would create gaps for them via suitable actions which deliver the system benefit. On the other hand, any action for the merging operation should minimally disrupt the mainline traffic condition. To address the problem of automated merging of CAVs, this research provides a method to help connected vehicles merging onto a connected environment. CAV operations and mobility data are still yet to be seen nationwide, hence, microsimulation of the environment is the accepted tool available to model and analyze the 
performance of any designed traffic management strategy. The Highway Capacity Manual (HCM) provides different traffic inputs for finding the capacity of the merging area. For measuring of effectiveness (MOE) of the proposed model, the traffic performance of the model (with its individual inputs) is compared to the traffic performance of the current day traffic under similar demands and input conditions.

While platooning will be commonplace in highways for increasing capacity and minimizing delays, many vehicles would require traveling on the non-platoon lanes due to reasons such as desiring to leave the highway from an onramp, lacking vehicular technology etc. Due to these reasons, in a multi-lane highway, the dedicated platoon lanes will be traveling on the left side lanes to have the least disturbance from onramp or off-ramps. With this configuration, any merging vehicle from the onramp has to safely and smoothly merge onto a non-platoon lane. The model proposed in this research provides a detailed merging algorithm for merging of onramp vehicles onto a mixed flow connected highway.

\subsection{Research Objectives}

The research focus is developing a merging algorithm which assists onramp connected vehicles merging onto a highway comprised of platoon lanes and a non-platoon lane adjacent to the onramp. The algorithms are designed based on the communication between the mainline and onramp vehicles and the infrastructure facility. The existence of a centralized controller in the merge area is considered in order to detect the appropriate control actions based on the traffic status and specify selected vehicles executing the action. The non-platoon lane is comprised of connected vehicles and the platoon lane has platoons with varying lengths which are equipped with CACC technology.

The merging process includes alternative actions such as deceleration and lane changing. Different alternatives aim to widen the merging gaps and sync them with the movement of the merging vehicle so that a smooth arrival to the auxiliary lane is provided. Consequently, with the system performance more vehicles can be expected to merge and with less delays. According to the gaps in the research, the specifications of this research for a centralized merge control and the set of control actions were designed.

For obtaining the goal of establishing an automated merging algorithm, the objectives of this research can be classified as: 
1) To develop an automated merging algorithm which utilizes the communications between vehicles and the infrastructure for merging, 2) To design a microsimulation model with the proposed merging model for CAVs in a multi-lane highway and compare with a base model, 3) to integrate the automated merging algorithms and evaluate their efficiency under different traffic conditions.

The contributions of the objectives will be grouped to a) an advanced traffic management solution for future merging areas, b) contribute to the general behavior of CAV technology and their benefits in highway operation, and c) to present a robust and efficient application of microsimulation for CAV traffic.

Within the scope of the research, the outcome presents the necessary traffic behavior, conditions, controls, architecture and infrastructure required for the setup in an actual merging intersection of a connected automated highway.

\subsection{Scope}

This research presents an automated merging algorithm which incorporates the connectivity between vehicles and infrastructure as a basis for the design of a merging policy within a multi-lane highway. The benefits of this model include providing a sound basis for CAVs merging onto a connected highway. The algorithm is evaluated for its performance in a verified microscopic traffic simulation environment which is comprised of platoon and non-platoon lanes. Because of the complexity of merging and actions requiring joining platoons, various traffic measures for the mainline and the onramp are considered. The simulation results are then analyzed to obtain the sensitivity of each parameter and achieve the best performance of the automated merging controller.

\subsection{Contributions and Dissertation Outline}

The contributions of this research are listed below:

1- The creation of a model for the simulation of a connected merge area environment

2- Proposing a new merge model for improving the merge area throughput

3- Analysis and evaluation of the traffic performance of the presented model for the merge area improvement 
The manuscript of this dissertation will include four chapters. Chapter 1 will describe the background, problem statement, research objectives, and the scope of research. Chapter 2 presents the current state of the research on CAV in highways, CAV technologies, and merging models onto highways. Chapter 3 explains the design of the automated merging algorithm, microsimulation modeling of the merging, and the analysis of the relevant parameters. In the end, Chapter 4 discusses the performance of the merging model, the benefits, the limitations, followed by suggestions for future research in this area. 


\section{LITERATURE REVIEW}

Connected Automated Vehicles (CAVs) will be a commonplace in the traffic in the coming years. Technologies of these vehicles are advancing swiftly, and numerous researches are conducted to study their effects on highways and urban areas. Technologies such as Adaptive Cruise Control (ACC) and Cooperative Adaptive Cruise Control (CACC) besides inter-vehicular connectivity will be utilized by CAVs to enable higher capacities and better traffic performance. One of the designs for future highways and freeways which is under investigation for yielding better traffic performance is a separate lane which will be dedicated for the CAVs to form platoons and travel with higher speeds and lower headways than the manual cars, similar to the High Occupancy Vehicle (HOV) lane available at present time (we will call that lane, as platoon lane) (Tiernan, Richardson, Azeredo, Najm, \& Lochrane, 2017).

Because of the connectivity between vehicles in a platoon and the autonomous driving with technologies such as CACC which CAVs will be benefiting from, the platoon lane is expected to have higher capacity rates and less delays compared to the regular traffic lane passing manual cars or mixed traffic (Lioris, Pedarsani, Tascikaraoglu, \& Varaiya, 2017). In current day highways the capacity of each lane fluctuates between 2000-2500 vphpl which is limited by the human driver perception-reaction time and the speed limits. In the platoon lane, vehicles will be driving with short headways and potentially higher speeds in platoons. These circumstances will yield higher capacities than current recorded capacities without any compromise to the safety or comfort of the passengers (Jones, 2013). Vehicle-to-Vehicle communication (V2V) and driver assistance systems such as Cooperative Adaptive Cruise Control (CACC) Collision avoidance are technologies which will provide the platform for the platoon formation.

For a better understanding of the connected highway merging concept, recent literature on CAVs, connected-automated highways, and merging controls are investigated. CAV technologies will eventually be enabling a more advanced and safer driving via improved reaction times, reducing human errors and enhancing decision making within the traffic flow. The CAV traffic has the potential to lead to fewer crashes and a more efficient transportation (Clements \& Kockelman, 2017). Merging on highways has a complex nature, thus making the phenomenon's modeling a challenging problem. Merging to highways and freeways with higher speeds is recognized as an accident-prone maneuver, which can easily perturb the traffic conditions in the merge influenced area. 
Throughout the past decades, different transportation management strategies such as ramp metering, variable speed limit, shoulder user etc. were tested to lower the delays and improve highway conditions. But since their traffic was operated by manual drivers, the merge area could not be controlled with any direct system other than ramp metering to ensure the safety and operation of merge areas. However, with the advent of CAV technology, the possibility to control the merge maneuvers efficiently has become possible. The CACC technology integrated into highways can double freeway capacity by the frequency of shorter following gaps (Nowakowski, O'Connell, Shladover, \& Cody, 2010).

The capacity of a platoon lane depends on the inter-vehicle headway and the inter-platoon headway. The inter-vehicle headway is the time headway between vehicles in a platoon and the interplatoon headway is the time headway between two consecutive platoons. Several strategies can be defined for the platoon formation. A CACC vehicle platoon is comparable with a train in which the first vehicle in the platoon acts as the locomotive, and the other vehicles follow the leader (Zhao \& Sun, 2013).

\subsection{Connected Automated Vehicles Technology}

Traveling with automated vehicles (AV) which can utilize the roads more efficiently and with less travel times, can lead to more frequent and longer trips (Fagnant \& Kockelman, 2015). The outcome of CAVs in traffic operations can re-produce the safety, throughput, flow stability, and flow breakdown (Talebpour \& Mahmassani, 2016). Higher accessibility to AVs can increase traffic demands which may add to the traffic congestion unless ITS based technologies assist in regulating high traffic flows. Therefore, the necessity of studying macro and micro scale solutions to solve future traffic regulations and issues. Travel time estimation and route choice will be revolutionized through the mass data or the Internet of Things (IoT). In the microscale level, maneuvers such as merging, lane change, overtaking etc. will be optimized by the mass data and high-resolution communication between all users of the infrastructure (Bevly et al., 2016). As an example, in the case of vehicles merging onto a connected highway, the merging action would be implemented by the controllers such that there is a trade-off between the merge capacity and the throughput of the highway's mainlines.

Research has found that in certain bottleneck points such as merging areas, if drivers rely on the connected system rather than their judgment, there is a better chance of controlling traffic (Gereon Meyer, 2014). The same idea is also applicable to urban intersections, off-ramps, etc. (Haneen Farah, 
2018). Currently due to the absence of connected highways, data collection is not possible for the new technology, making analysis tools limited to test track, simulators, and traffic models.

Microsimulation has been extensively used to model and evaluate the effectiveness of applying CAV technology in recent years (Xie, Zhang, Gartner, \& Arsava, 2017). Traffic studies for the upcoming technologies can yield the foresight for the emerging ITS operations.

CAVs will be able to send Basic Safety Messages (BSM) through Dedicated Short Range Communications (DSRC) to enhance safety and enable faster and safer maneuvers (Board, National Academies of Sciences, \& Medicine, 2017). Currently, with Driver Assist Systems, vehicles receive alerts and information but not the driving tasks performance. However, it is anticipated that full automation (coming after conditional automation) will control all driving maneuvers relying on the real-time information (DOT 2016).

Hu and Sun (2019) developed an online system control algorithm for multilane freeway merging areas within a CAV environment based on optimizing vehicles' lane changing and car following trajectories. A Cooperative Lane Changing Control (CLCC) optimization model was proposed to ensure safe and smooth lane changing execution. Their proposed model was tested in a two-lane highway and their results displayed lower delays compared to existing merge conditions.

\subsection{Cooperative Adaptive Cruise Control and Platooning}

Some researchers have looked at how platoons can be formed and the technical details behind vehicles maneuvering into platoons (Kamali, Dennis, McAree, Fisher, \& Veres, 2017; Karbalaieali, Osman, \& Ishak, 2018; Michaud, Lepage, Frenette, Letourneau, \& Gaubert, 2006). The maneuvers for lane change and merging by CAVs in different scenarios (related to platoons) having cooperative adaptive cruise control and vehicular communications has been conducted (Bevly et al., 2016). Rahman et al., evaluated the car-following behavior models for cooperative adaptive cruise control systems considering user comfort and safety within a parameter called "user acceptance"(Rahman, Chowdhury, Dey, Islam, \& Khan, 2017). Larson et al., looked at how directing platoons of CAVs with the same destinations can be beneficial on time while using the least amount of fuel (Larson, Munson, \& Sokolov, 2016). Research has been implemented which viewed the platooning from the perspective of the passengers in a vehicle within a platoon and analyzed the safety and comfort factors (Jones, 2013). 
Milanés et al. (2014) studied the effect of CACC on traffic flow and on highway capacity from results obtained from a test track data. The authors presented a CACC system formulated on gap regulation and gap closing controllers. The Intelligent Driver Model (IDM) was applied to model the car-following for vehicles. The speed variation for cars with CACC was lower compared with that of ACC. The traffic flow stability also improved resulting from smoother and more stable reactions (Milanés et al., 2014).

Öncï et al. (2014) considered CACC for the evaluation of a network system. The effect of CACC on the traffic flow performance measures were studied from results within a test track. This research concluded the necessity of CACC for platoons with enhanced communication (Öncü, Ploeg, Wouw, \& Nijmeijer, 2014).

Milanés and Shladover (2014) developed the Intelligent Driver Model system with the features of ACC and CACC controllers. Their experimental results were obtained from four vehicles to understand the car-following in test tracks. This model was designed to manage car following for the leading vehicle in a platoon and to regulate the joining of platoons. The results of test track experiments displayed how connectivity improved the flow of commercial vehicles (Milanés et al., 2014).

Amoozadeh, Deng et al. (2015) investigated the platooning formed through CACC and based on the vehicular ad-hoc network to control acceleration for free flow and collision avoidance through constrained and emergency braking. The VENTOS which is an integrated simulation platform was used for joining and leaving maneuvers in platooning. Several platooning scenarios were implemented in the simulation model to show the validity and effectiveness of the approach.

\subsection{Capacity increase via platoon lanes and CAVs}

One of the major expectations from CAVs and platooning has been their positive impact on increasing the road capacity and mitigating the congestion problem. Fernandes and Nunes looked at an algorithm which considered vehicular cooperation and leader's positioning on the capacity of a highway (Fernandes \& Nunes, 2015). The US Department of Transportation have done experiments on platoons with vehicles equipped with adaptive cruise control and DSRC communication setting the federal frameworks for the prospective platoon formation by CAVs and concluded that with the successful short headways between CAVs, higher capacities can be expected by platoon formation 
(Jones, 2013). Zhao and Sun simulated the lane capacity with platoons of CACC equipped vehicles and found that with the increase in the CACC market penetration rate, capacity increases significantly unlike platoon size which has little impact on the capacity (Zhao \& Sun, 2013). Lioris et al., studied how throughput of urban roads can be increased via CAVs and concluded that a double capacity can be achieved by platoon formation in urban roads (Lioris et al., 2017).

The focus of the current research of CAVs in highways, is integrating vehicle autonomous technologies and cooperative driving based on V2V and V2I communications. The CAVs will be directed gradually to improve traffic safety and efficiency, besides delivering lower fuel consumptions. Research estimates show that CAVs can reduce the traffic costs by an amount of $\$ 1.2$ trillion per year only in the States (Clements \& Kockelman, 2017).

An advanced merging controller system can potentially overcome the capacity shortage on merge areas of highways when connectivity between vehicles and infrastructure is available. The following sections in this literature review includes overviews of the different aspects of CAV's features, automated merging, and platooning.

The research has displayed the effectiveness of CACC and its potential coupling with platooning in improving traffic flow performance measures including throughput, travel time, and string stability. As researchers sought specific outcomes from these technologies, they have focused on the potential improvements in limited or smaller scale experiments within test tracks or microsimulations.

\subsection{Automated Merging Control- Current Research}

Numerous researches have proposed merging control algorithms for cooperative merging algorithm application. Different aspects of lane change and merge maneuvers of CAVs have been studied to explore the capabilities of the new technologies aiding the transportation systems (Bevly et al., 2016). Tsugawa et al. (2001) studied the feasibility of cooperative driving within a connected environment. Within a test track, platoons of 5 vehicles with speed range of 24.8 to $37.3 \mathrm{mph}$ were tested for the research. The intra-platoon gap distances in the testing were 66 and 111 feet in a singlelane and two-lane tests, respectively. The study showed the successful lane changing and merging of vehicles in the platoons (Tsugawa, Kato, Tokuda, Matsui, \& Fujii, 2001). 
Ahmed et a. (2018) Vehicles equipped with V2X capable OBUs transmit basic safety messages (BMSs) every tenth of a second. A BSM packet is comprised of a vehicle's identifier, GPS positions, speed, time, and direction. The freeway merge assistance system utilizes these BSM packets and determines the optimal merging order for drivers by applying a 3-way handshaking communication protocol between the onramp and mainline vehicles. Lacks group merging and platoons in mainline (Ahmed, Hoque, Rios-Torres, \& Khattak, 2018).

Chen et al. (2018) merging model used MPC and a cost function. Though there research did not consider any platoons or multi lane highways, they formulated the merging as a control problem (Na Chen, 2018).

Hussain et al. (2020) designed a cooperative Nonlinear Model Predictive Control (NMPC)based optimization method for the execution of a highway lane merge comprised of two connected autonomous vehicles using solutions obtained by the direct multiple shooting method. Their results showed the possibility of the operation of this design considering the connectivity between vehicles.

Subraveti et al. (2018) assessed a rule-based traffic control strategy at a motorway merge aiming at reducing travel times. The rule-based system advised vehicles from the control system influencing the longitudinal behavior of mainline vehicles. The methodology was applied to a case study via microsimulation, which the results found that at high penetration rates, a slight reduction in TTT (1.9\%) was found but no effect was observed at lower penetration rates (Subraveti, Knoop, \& Arem, 2018).

Shen et al. (2018) devised a heuristic based optimal control algorithm for a merge area onto a highway. Their model was based upon efficient control of the lateral and longitudinal movement of vehicles in the merge area in order to make lane change decisions. With numerical simulations, they displayed the efficiency of the proposed planning algorithm over simple sequential planning policy (Shen, Hu, Sun, \& Deng, 2018).

Omidvar and Pourmehrab (2018) proposed an optimization algorithm for freeway operations at merge zones which targeted the maximization of the average speeds. Their system receives arrival information as inputs and generates optimal trajectories for AVs while predicting the behavior of conventional vehicles. The Objective is to maximize speed in merge area. The results of the research showed that a minimum of $25 \% \mathrm{AV}$ penetration rate is required to observe improvements in operational conditions (A. Omidvar, 2018). 
Rathgeber, Winkler et al. (2015) formulated a model for the estimation of the trajectories of AVs based on a nonlinear model which incorporated lane changing and emergency braking. Their conclusion was that trajectory planning and control in the driver assistance system would be necessary to guarantee safety and comfort (Rathgeber, Winkler, Kang, \& Müller, 2015).

Rios-Torres et al. (2017) developed an analytical closed-form merging solution for CAVs' fuel consumption. In their framework, one vehicle merges at a time, while the speed in the merge area is constant. Several simulation experiments in MATLAB/Simulink were performed for sets of 2 and 15 vehicles in each road with different constant simulation speed values all in the range of $24 \mathrm{mph}$ to 64 mph. They tested their model against the baseline scenario in which the mainline has the right of way in CAV environment. When the number of vehicles on the mainline increases, merging vehicles are forced to decelerate or stop (Rios-Torres \& Malikopoulos, 2017).

Ntousakis, Nikolos et al. (2016) presented a merging assistance model which applied longitudinal trajectory planning for a pair of vehicles at a merging junction. The paper aimed at minimizing acceleration and jerk rate via using a discrete time quadratic programming formulation. The model predictive control considered a fixed merging point downstream which every merging vehicle's final speed and time of arrival to the point could be determined. The simulation of six merging vehicles with predefined sequences and constant speeds was performed for the evaluation of the proposed model(Ntousakis, Nikolos, \& Papageorgiou, 2016).

While these aforementioned studies provide useful insight into several algorithms supporting the merging assistance application of the CAV technology, they do not account for the various traffic conditions in the evaluation of such algorithms. On the contrary, other studies account for traffic conditions in designing and testing several merging assistance algorithms using simulation in connected vehicle (CV) environments. For instance, a lane changing advisory algorithm was developed by Park, Bhamidipati et al. (2011) based on V2V and V2I communications. The proposed algorithm was tested for different safe gap values (small, medium, and large) using Vissim simulation and took into consideration mixed traffic of connected cars and trucks in a two lane non-platooned freeway. The algorithm was tested in a simulation of 15 minutes with demand values of 2,774 and 613 vehicles per hour (vph) for the mainline and on-ramp traffic, respectively. The study showed that while the choice of small gaps resulted in marginal improvement in the mainline operational condition, medium gap improved the average mainline speed by $6.4 \%$. When implementing the large safe gap, the improvement in the average speed decreased to $2.2 \%$ (Park, Bhamidipati, \& Smith, 2011). 
Marinescu, Čurn et al. (2012) developed a slot-based merging algorithm using V2I which functioned based on lane change advisory messages sent from a roadside unit (RSU) to mainline vehicles in order to assign gaps with merging vehicles. In a VISSIM simulation model, the proposed model was tested in a multi-lane freeway with medium to heavy mainline traffic comprised of 3,600 $\mathrm{vph}$ and 4,700 vph, demands respectively. The different on-ramp traffic demands of 200 to 2,000 vphs were tested. The proposed algorithm's performance was evaluated through comparison to the Vissim's human driver model. The results showed that the slot-based algorithm outperformed Vissim's human driving model through improving the travel time and reducing delay of merging vehicles. This study indicates promising potential for cooperative merging algorithm in a connected environment; however, the proposed algorithm did not include platoon traffic. Applying the Vissim's human driving model without any modifications to consider for AV technology is part of this work which can be developed. Noteworthy is, that the mainline traffic conditions were not evaluated under the application of the cooperative merging assistance application (Marinescu, Čurn, Bouroche, \& Cahill, 2012).

More recently, Xie et al. (2017) developed an optimization-based ramp control strategy in an integrated platform of VISSIM, MATLAB, and the Car2X module. The ramp input demands ranging from of 300 to $700 \mathrm{vph}$ and the mainline from 800 to 1,200 vph were simulated. The results displayed that the controller effectively managed merging actions, having the limitation that the traffic is not oversaturated at the merging junction and that there are no platoons of CAVs. The results of this research show that the proposed optimal control strategy can effectively coordinate all merging vehicles at freeway on-ramps while improving safety and efficiency (Xie et al., 2017).

Most merging controllers for CAV environments are investigated for one vehicle's merging at each time, and there are fewer studies addressing the platoon merging (Scarinci \& Heydecker, 2014). The concept of adopting the merging point to estimate the arrival time of a merging vehicle from an onramp onto the mainline is used in several research studies ((Dong, Dolan, \& Litkouhi, 2017) (RiosTorres \& Malikopoulos, 2017), (Xie et al., 2017)). The results of this research present the significant role of communication parameters on the platooning performance measures (Amoozadeh, Deng, Chuah, Zhang, \& Ghosal, 2015).

In a more in-depth study, Zhong, Lee et al. (2017) investigated the features, mobility, comfort, safety, and fuel consumption in platooning formed by CACC. This research developed an optimized longitudinal controller model. The best performance of the designed model was achieved with headway values ranging from 0.6 to 0.9 seconds. The CACC formed platoons reduced time headway 
deviation and the instantaneous fuel consumption by $98 \%$ and $33 \%$, respectively, compared to a nonoptimized base model. Altogether, the effect of ACC and CACC controllers based on speed difference and minimum safe gap have been studied thoroughly in different microsimulation models (Zhong, Lee, \& Zhao, 2017).

Higher traffic capacities achieved through high speed platooning aimed at responding to larger demands could be one of the worthiest outcomes of CAVs merged with platooning and is extensively assessed by simulation models. The simulation results can first disclose the operations of the future's traffic and be exploited for evaluation of network operations. There are many details of the control methods devised for integration with CAVs such as lateral and longitudinal controls, vehicular dynamics, automated merging, and diverging that should be investigated in advance before deployments. Therefore, there is a large domain of benefits and challenges of CAV which are important to investigate at the current stage of these innovations. In this regard, the required infrastructural systems to support automated highways, advanced traffic controls, and advanced management methods to implement are the research necessitates to understand their opportunities and constraints prior to the high level of penetration of CAVs in the traffic. Table 1 displays a summary of relevant research studies in proposing a merge strategy for onramp vehicles onto highways. 
Table 1 An overview of the major research studies in onramp merging

\begin{tabular}{|c|c|c|}
\hline Author & Year & Methodology \\
\hline Marinescu et. al., & 2012 & $\begin{array}{l}\text { A roadside unit identifies a slot and assigns it for } \\
\text { each merging vehicle }\end{array}$ \\
\hline Karbalaieali et. al., & 2018 & $\begin{array}{l}\text { Merging action on a fully platoon operated highway. } \\
\text { Control action selected based on the minimum travel time } \\
\text { estimated for the merge area vehicles }\end{array}$ \\
\hline Subraveti et. al., & 2018 & $\begin{array}{l}\text { Rule based control actions to create gaps for merging } \\
\text { vehicles }\end{array}$ \\
\hline Rios et. al., & 2017 & $\begin{array}{l}\text { Merging solution model for CAVs merge with the } \\
\text { objective of minimizing fuel consumption. One vehicle } \\
\text { merge at a time, while the speed in the merge area is } \\
\text { constant. }\end{array}$ \\
\hline Shen et. al., & 2018 & $\begin{array}{l}\text { Heuristic model controls the lateral and longitudinal } \\
\text { movement of vehicles in the merge area in order to make } \\
\text { lane change decisions. }\end{array}$ \\
\hline
\end{tabular}

The literature review addressed different aspects of merging to investigate CAV potential in the efficient merging models. Yet more aspects of CAV merging remain to be explored.

Figure 1 displays the contribution of this research in filling the gaps of the literature. Numerous research studies have been implemented on CAV technologies and cooperative merge strategies. But 
the current literature is missing study on the design of a merge control actions when there is a mixture of platoon and non-platoon lanes. Additionally, the simultaneous effect of highway geometry, traffic behavior, and merge strategy has not been investigated.

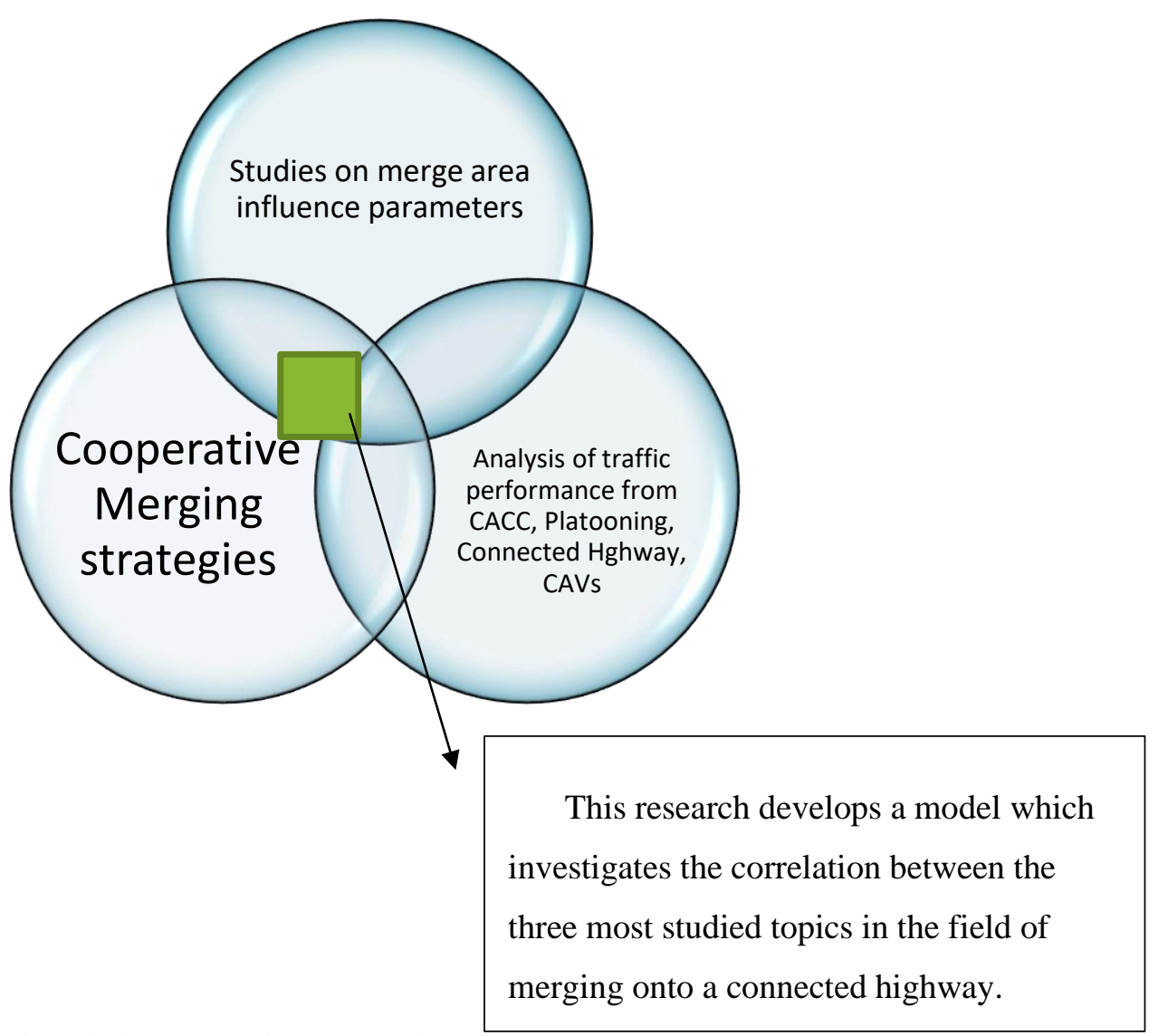

Figure 1 Previous research and the aim of this research

This study aims to overcome some of the shortcomings in previous studies by developing a RuleBased merging control algorithm in a connected environment with two main targets; assisting onramp vehicles in merging smoothly and safely, while maintaining the mainline conditions with the least effect of adverse disruptions from merging vehicles. Further contribution of this research is the analysis of effective parameters for the efficient merge according to the control strategy introduced in the study.

The novelty of this research is the creation of a model which can be applied for the traffic simulation of a multilane highway with mixed flow (platoons and non-platoon lanes) and a control strategy which is tested for an efficient merging of onramp vehicles. In doing so, several experiments for different traffic conditions and merging setups were designed. The developed scenarios are evaluated in the traffic microsimulation model. 


\section{METHODOLOGY}

\subsection{Merge Area Influence}

The merge area influence is identified to be the segment close to the merge ramp in which the traffic flow behavior changes due to the merging action. The HCM has introduced guidelines for measuring the capacity of the merge area according to relevant parameters influencing the merge area, such as length of acceleration lane, number of lanes, width of lanes etc. Figure 2 displays one of the common geometries used for merging with an acceleration lane to a multilane highway. As can be seen in the picture (Handbook 1998), the merging influence area covers a section of the upstream and downstream of the mainline traffic. More number of lanes in the highway can assist in reducing the potential disruption of merging vehicles, though they can additionally lead to unnecessary lane changes in the merge area.

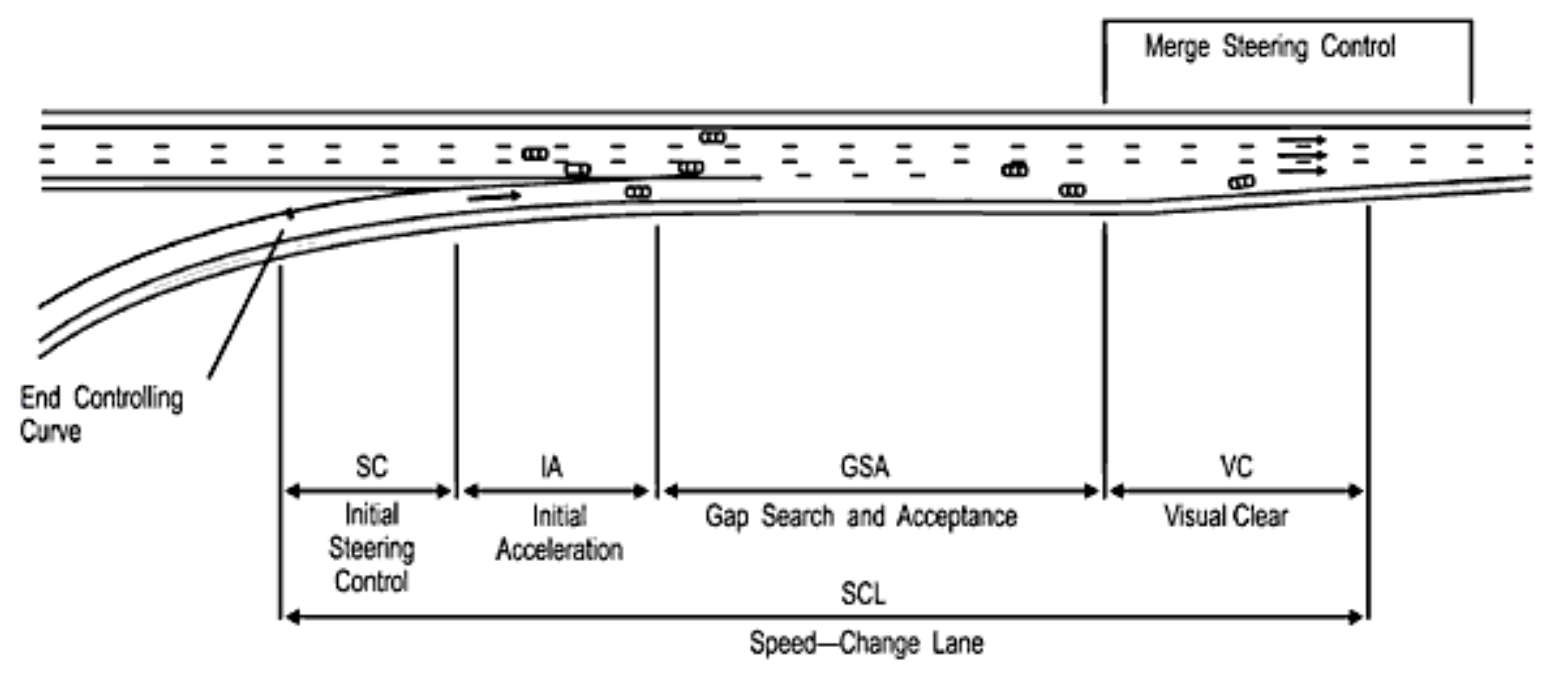

Figure 2 Onramp merge section from the FHWA handbook ${ }^{l}$.

\subsection{Control System Features}

A control model for the improvement of the merge area is one of the well-known solutions in the traffic engineering world. One of the control methods for merge onto highways is ramp metering

\footnotetext{
${ }^{1}$ https://www.fhwa.dot.gov/publications/research/safety/97135/rs2.cfm
} 
which intends to prevent large traffic volumes from entering an already congested highway. Controls can now be devised to be more advanced due to the communication possibilities between vehicles and the infrastructure.

For creating a control model for the assessment or analysis of a specific area, many different aspects should be initially investigated before the design and implementation of the model. The primary considerations for the model are depicted below:

\section{A. Identification of the problem}

The first step for designing a control system is the analysis of the traffic problem that needs to be solved. In this research the aim is solving the congestion at merging areas which occur due to the conflicts between the traffic flow on the mainline and on-ramps. In the current day traffic, lack of sufficient gaps for the merging vehicles leads to either forced merging where ramp vehicles execute a forced lane changing maneuver causing vehicles on the mainline to decelerate rapidly which may lead to disturbances on the mainlines. Thus, it is important to identifying the factors that can be controlled which can yield a better merging process. Certain parameters of the merge area such as extremely abnormal merging and mainline demand, poor weather conditions, spillbacks from off-ramps are not part of this study. This research focuses with the design of a control systems that assists the merging process. Considering this, the parameters that can be controlled include the speeds of the vehicles, average headways, lane change decisions, accelerations etc.

\section{B. Selection of control actions for the merge}

The objective of the control strategy is creation of a sufficient gap for the on-ramp vehicle to merge at the right time delivering a safe and efficient merging. For assisting the merge, the mainline vehicles can be influenced either in the longitudinal or lateral direction for completion of an action which creates gaps and facilitates the merging process. Longitudinal movements can be controlled by a change in the vehicle's acceleration, while the lateral movement is in the form of a lane change. The modification of speeds includes change of desired speed etc. In terms of lateral control, in this research the aim is to potentially join a platoon of CAVs in the adjacent lane which must take into consideration factors such as traffic flow on the other lane, acceptance of the platoon, trade-offs 
between disturbances caused by lane changes and creation of gaps etc. The variables that can be controlled in the lane change of the merging vehicle include the timing of lane change, location, and the decision to change lane. Lateral movements involve more than one lane and require further sensitivity analysis of the conditions.

\section{Traffic state and measurements}

An important step in the formulation of the control algorithm is the identifying the relevant and required measurements to perform the necessary action. The real-time information of the traffic is usually obtained from loop detectors which can give traffic state via processing the data through estimation techniques. In this research, the connectivity between vehicles is also considered for the merge actions, while considering that vehicles and the infrastructure are all connected.

\section{Conditions for the operation of the control function}

It is important for the conditions of the control action's operation to be specified so the scope of the research is understood. With the determination of the scope of the study, the creation of the desired scenarios for testing the action and analyzing the performance of the design will be structured. Therefore, it is important to understand the problem, the frequency of occurrence and the effectiveness of the control in solving the problem. A control action may work for freeflow conditions or congested conditions or both, therefore the design should be stable for the intent of the study and robust in changing factors within its accepted domain.

\section{E. Assumptions and constraints}

For any control system, certain assumptions are considered which have to be realistic and according to engineering principles. A fully connected highway is an assumption used in this research. Another example of an assumption in this research is, gaps created using the control action, will be reserved for a merging vehicle and cannot be occupied by other vehicles. 


\section{F. Location/timing of the merge action}

The appropriate location or timing of an advice leading to a control action should be acceptable in terms of the possibility of the action and the outcomes yielded from the action. Any action for creating gaps should be considering the required safety measures, comfort acceleration or deceleration, maintaining minimum safety gaps between following vehicles etc. As an example, a gap should not be created at a time when the merging vehicle is very close to finishing the acceleration lane.

\section{G. Performance Indicator}

For the analysis of the outcomes from the control action, the right performance measures should be selected and analyzed. In this research the aim is obtaining maximum throughputs for the merge area while minimizing the travel time and having the least number of actions for the merging vehicles.

The model created will be a multi-lane highway with platoon and non-platoon lanes in which onramp vehicles will intend to join the lane 1 which is a non-platoon lane. The configuration of lanes is such that platoon lanes will be the left most lanes and non-platoon lanes will be the right lane where vehicles can have the ability to leave the highway without causing perturbation in the platoons.

\subsubsection{Centralized Merging Controller}

Merging at highways is a challenging task which incorporates the action of one or several vehicles in order to be completed safely. This task can become more complex with the existence of specific platoon lanes. The technology of CAVs enhanced by connectivity can aid the merging action by eliminating the drivers' judgment and perception of speed, and gap. The merge action (of current day traffic) which relies on the right of way for the mainline vehicles and available gaps for the merging vehicles is incapable in yielding the maximum potential capacities of a merge area and insufficient in managing large simultaneous demands on the onramp and mainline. In order to overcome the limitations of current merging areas, there is the challenge of how required gaps for merging could be deliberately created. This creation of gaps must be precise, systemic, safe, and made by the actors on the road. The requirements for such a system are, the receiving and processing of the 
road information by a controller and sending the required actions to be implemented by the determined actors.

One of the architectural designs for the merge area controller is the existence of a central controller which would receive the merge area traffic information, process the information and send the necessary action-information to specific actors of the merge action. In such a design architecture, the central controller is part of the infrastructure which monitors the traffic and sends information on a real time basis. The advantage of a central controller is that it can eliminate the randomness in individual vehicle's car actions for a merge event and its selected actions can be based upon a broader length of the traffic flow-thus yielding system benefit-compared to actions selected by vehicles in proximity of each other. The centralized controller is assumed to have real time information of the traffic via the fastest means of communications e.g. $5 \mathrm{G}$ to provide required info for the merging process.

Different merging actions produce different traffic performances in the merge area. The differences in performance can be in the overall and average travel times of vehicles in the merge area, the average speeds in the merge area etc. Thus, in order to obtain higher throughputs in the merge area, there is need for minimizing the delays of this area. A systematic model which manipulates vehicles' movement in the merge area-with the aim of minimizing human-related delays-could enhance the merging. To identify the appropriate decisions in the controller, it is necessary that the controller choose the best possible approach that reduces delay and ensures safety. The threshold for the controller's operation should be identified according to the traffic condition on the network. Specific Measurements of Effectiveness (MOE) such as average speeds and travel time in the merge area could assess the necessity of the merging assistance from the controller including speed and travel time along the road.

\subsection{Research Aim and Contribution}

\section{Research Problem}

In the current network of multilane highways, the merging sections have lower throughput compared to the straight sections of the highway. This phenomenon is mostly due to the manual decision making and merging action by vehicles in this segment which don't not efficiently utilize the available gaps on lane 1 (rightmost lane of the highway) and potential gaps on lane 2 and 3. 
According to HCM, the average capacity of non-merge sections of highways are 2000-2500 $\frac{\mathrm{veh}}{\mathrm{hr}}$, which this rate drops $15-20 \%$ in the merging sections.

With CAVs, the capacity of basic highway as well as merge sections is expected to significantly increase. While there has been some previous research on cooperative merge, there is limited work on how merge sections could be controlled and operated with CAVs traveling in platoons on the mainline lanes.

\subsubsection{Research Objective}

The main objective of this dissertation is to investigate how CAVs (Connected Automated Vehicle) could improve the operations of a merging section onto a connected automated highway. This research aims at developing an advanced Rule Based automated merging algorithm which delivers a safe, efficient, and robust merging model for a multi-lane connected highway comprised of platoon and non-platoon lanes. The basis of the merging algorithm is providing the maximum throughput of the merge area via the controlling mechanism which is a type of a Rule Based system. The novelty of this model is the development of a control system for assisting the merge in the highway configuration incorporating traffic of platoon and non-platoon lanes. The application of the Rule Based model enables different circumstances to be assigned an appropriate action, thus providing a thorough control over the range of possibilities which can occur in the system. Another advantage of Rule Based control is the selection of the correct diagnosis based on the system (traffic conditions). This type of control in this research is directed towards controlling the merge based on the traffic status.

One of the contributions of this research is the analysis of how the merge control strategy, different traffic flow behavior and the geometry of the merge area, can independently and dependently affect the merge area capacity of connected highway environment with a centralized controller. This would require simulations to enable a calibrated and validated result of the control strategy.

The current objectives and goals of this research are listed below.

Goals:

- Create a valid microsimulation model which incorporates all the required features for a connected multilane highway comprised of platoon and non-platoon lanes

- Design and implementation of the control algorithm for the merging of vehicles 
- Formulate relevant control strategies for both on-ramp and mainline vehicles that are adaptive to system state: the control strategy is developed upon the safety, vehicle dynamics and time efficiency

- Understand the effectiveness of control actions for merging in different state-action scenarios of the automated highway: Calibrate the relevant parameters in the merge control system to obtain the best performance compared to the base scenario

- Investigate the role of different model inputs on performance of the model

- Provide a merging model within a connected environment which can be further enhanced with specifications of new technologies for future studies

In order to complete this research, five main tasks and several sub-tasks are pursued:

1) Construct traffic microsimulation models representing CAVs

I. Vehicle input of platoons (with diverse size, speed, and headway) and non-platoon vehicles (diverse speed and headway)

II. Thresholds and variable parameters tested for verification of the simulation

III. Travel performance evaluation is coded in the simulation

2) Develop a framework for merging methods for CAVs in a connected environment relying on the real-time information dissemination.

I. To design an algorithm based on adaptive deceleration or lane change

II. To account for deceleration/acceleration on the onramp

III. To help connected vehicles split and generate acceptable gaps

3) Program the developed automated merging algorithm in the simulation model

I. Several safety conditions are realized to regulate the controller's performance

II. The alternative of deceleration and lane-change are executed 


\subsubsection{Research summary}

The problem which is investigated in this study is providing gaps for merging vehicles to a multilane highway-comprised of platoon and non-platoon lanes-via appropriate actions feasible on the mainlines and the onramp. The selection of controls is according to a rule-based model which activates an action ordered by the central controller when certain thresholds are met. The traffic states which the model in this study considers are uncongested conditions on the mainlines and onramps (platoon lanes have a very large capacity and will not be close to congested conditions).

The highway investigated in this research has 3 lanes. CAVs travel in platoons on Lane 2 and Lane 3 which are the two left lanes whereas the right lane serves the traffic of connected vehicles which are not within platoons. A microsimulation model is developed to test alternative control and car following options assuming that all vehicles are CAVs. In the model, a cooperative merge behavior is proposed for a three-lane highway where mainline vehicles in the upstream take actions (if necessary) to improve ramp merging and highway throughput efficiency. These actions are triggered if an onramp vehicle is unable to safely merge (and is projected to come to a stop towards the end of the acceleration lane) due to the present gaps on lane 1 being smaller than the minimum safe gap for merge.

The macroscopic results of this research include:

- Obtain the improved traffic performance in a merge area within a connected multi-lane highway compared to the base scenario

- Comparing the traffic performance between different actions assisting the merge

- Sensitivity analysis of the merge control parameters

\subsection{Operation of Merge Models}

When viewing the merging action in a connected environment, the factors which influence the merging action are, when, where, and how the merging will take place safely and comfortably. In other words, the effect of merge on the traffic performance of the mainline is dependent on the action characteristics. The characteristics of the action are dependent on,

- Type of action (Cooperative/non-cooperative merge) 
- Point of action initialization

- Type of information exchange

In this research, the differences above for the merging action will be reflected in three types of action. The basis of the models is to test all possible movements which can enable an onramp vehicle to merge onto a multi-lane highway within a connected environment which has dedicated platoon lanes beside non-platoon lanes.

The goals for testing three types of merge is understanding the traffic performance from the model, geometry, and the effects of actions effects on the merge area. Each merge type will be according to a specific logic and simulating the merging action under that logic. The three merge types modeled will each be simulating according to certain preferences. Merge type 1 involves onramp and lane 1 vehicles, type involves all lanes except lane 3 and type 3 incorporates all lanes in the merging process. In the next section, the details of the models are explained.

\subsubsection{Potential Actions to Assist Merging}

Merging onto the highway can be classified as a controlled merge or a non-controlled merge (replicating the current day merging). The controlled merge is a merge where the whole merge action or parts of the action are dependent on the information exchange with other vehicles or the infrastructure and executing actions accordingly. The non-controlled merge is when the merge is completed via the gap available for merging or cooperation between 2 individual vehicles. Therefore, in controlled merge, the connectivity is utilized for the completion of any merge action when the required gaps are not available.

A merge action will provide a gap large enough for the onramp vehicle to merge via certain actions. The actions for the controlled merge which will create a larger gap include:

- lane change, non-platoon vehicles joining platoons, platoon vehicles joining platoons etc.

- deceleration/acceleration, of a non-platoon lane vehicle (on lane 1)

The logic behind the actions for creating gaps (listed above) is the physical feasibility of these actions in the merge environment and the possibility of these actions via connectivity. Due to the connectivity between vehicles and the infrastructure, it can be assumed that in a connected highway, vehicles can join the platoon lanes and platoon vehicles can join the non-platoon lanes. 


\subsection{Model Development}

\subsubsection{Model Components}

The merge area traffic is a phenomenon comprised of numerous parameters, which include random and deterministic parameters ${ }^{2}$. Due to the nature of modeling such a phenomenon, obtaining an analytical formulation which covers all the random aspects of the reality is difficult. Thus, modeling with a heuristic approach is an accepted and suitable method for the analysis of a merge area with defined specifications. In this research the heuristic approach for the analysis of the merge area to a connected highway with CAVs is investigated.

In the proposed geometry of the highway which can be seen in Figure 2, the vehicles on the platoon and non-platoon lanes of the mainline and the onramp CAV traffic demand are the factors in forming the throughput in the downstream of the onramp. The architecture of the model is comprised of two different sub-models, the control model for merge and the traffic flow model.

The control algorithm triggers specific actions for assisting the merge if required. One of the major challenges in the creation of this model was the simulation of platoon lane and non-platoon lanes beside each other. This challenge was due to the complexity related to different traffic behavior of each lane. Instead of using regular market-available microsimulation methods, the $\mathrm{R}$ language was using for building the model. Creating the model from scratch prevented having to simulate from an external simulator. Figure 3 displays the main components of the merge model while Figure 4 displays the effective parameters on the microscopic and macroscopic setting of the model.

Numerous challenges must be overtaken for the verification of the model, including selecting appropriate geometric design, actions possible (in the presence of platoon and non-platoon lanes), calibration of car following parameters etc.

${ }^{2}$ The deterministic parameters in a merge area are also under the influence of randomness, as an example a deterministic car following model is under the random effects of average headways, speeds and desired gaps which are formulated for the model inputs. 


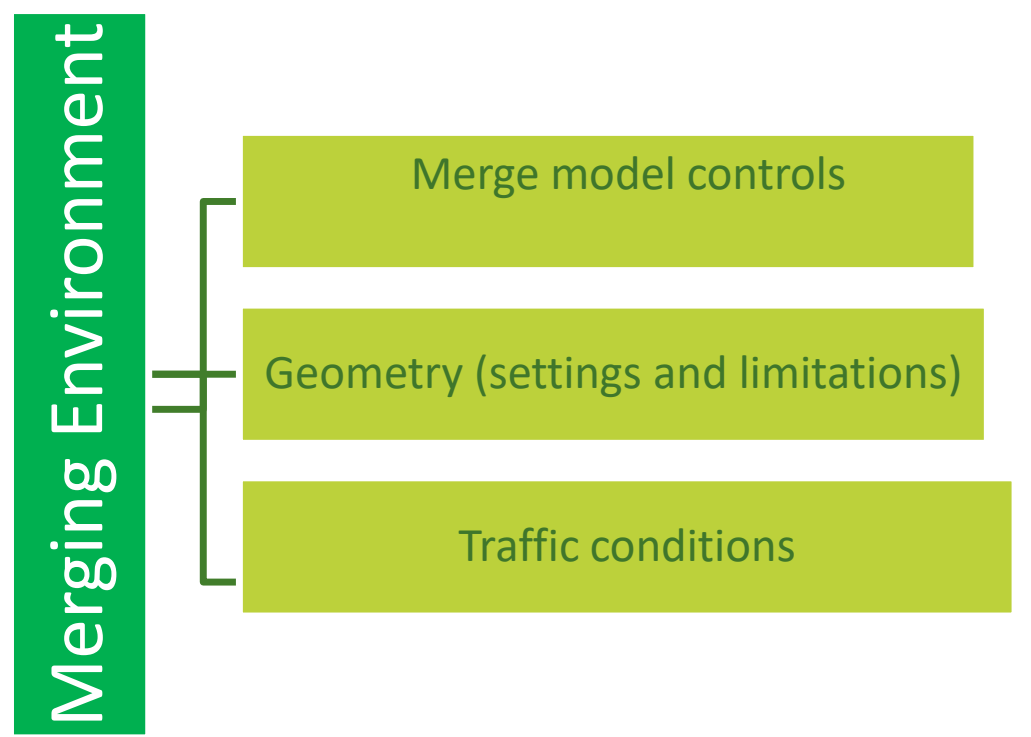

Figure 3 The components of the merging environment analyzed in the proposed model
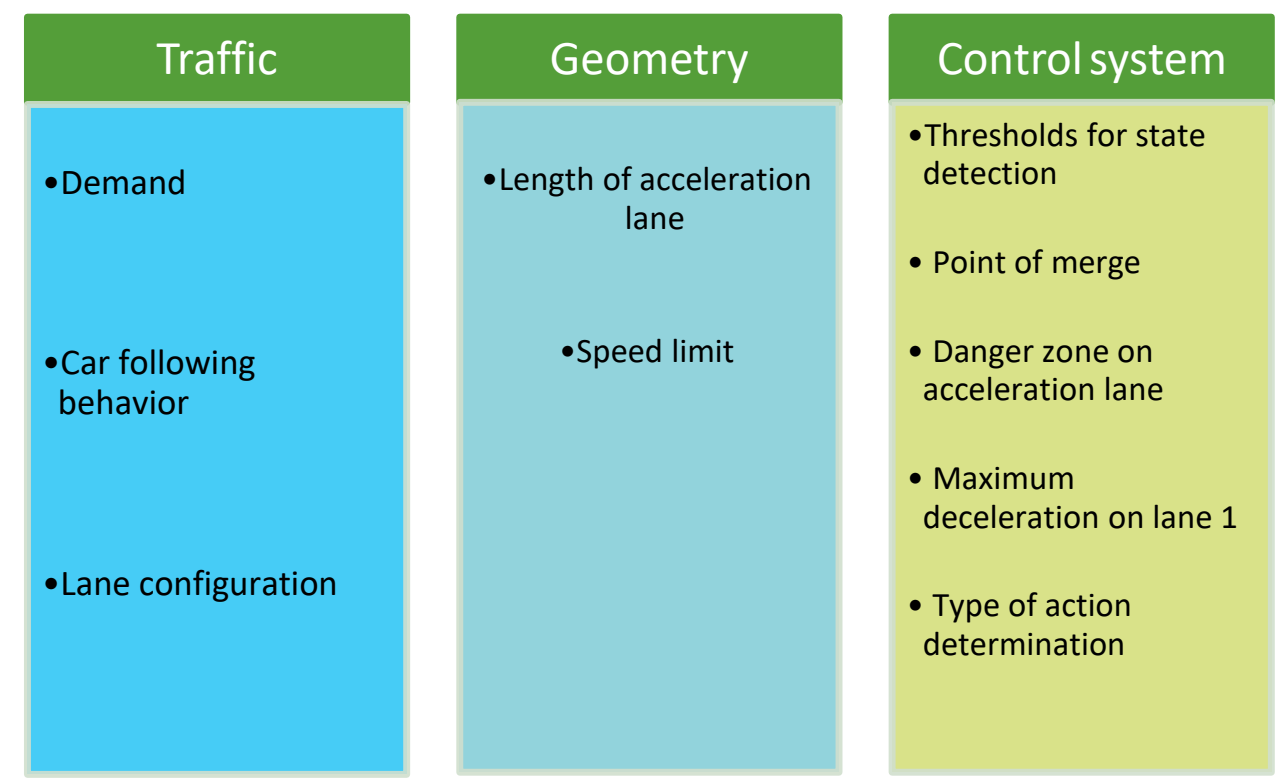

Figure 4 Major parameters in the model components

The model will utilize the analysis of macroscopic and microscopic features to obtain the results of the research questions. The macroscopic analysis will be conducted for:

- Analysis of traffic performance measures with different actions accommodating the merge in a connected highway comprised of platoon and non-platoon lanes.

- Analysis of the traffic performance of mainlines comprised of platoon and non-platoon lanes 
- Analysis of the role of geometry, speed limit, platoon length etc. on traffic performance

The microscopic elements programmed in the model include:

- Rule based specification of the merge action

- Car following model

- Lane change model

Figure 5 displays the macroscopic outputs and microscopic inputs of the model.

Within the framework created for the traffic of platoon and non-platoon lanes, the ability to configure the simulation and control algorithms enables testing the individual and accumulative effect of relevant factors on the model outputs.

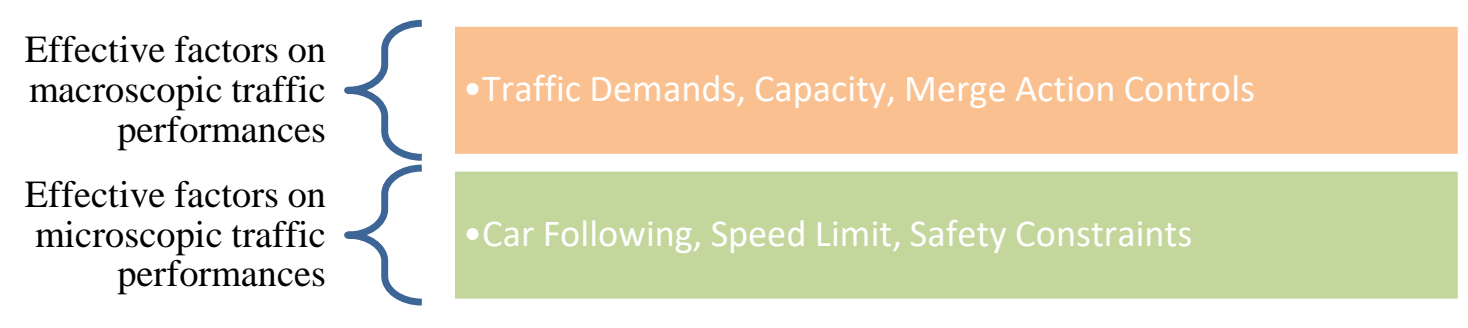

Figure 5 Effective factors on the macroscopic and microscopic behavior of model

The main attributes related to the model components are displayed in Figure 6. It can be summarized that the main features of the merging model traffic components are, the traffic flow attributes, the traffic demands, the car following models and actions for merging. The stochasticity in this model is presented in the demand and car following parameters. The deterministic sections of the model are the geometric and the control parameters. In the diagram below the parameters effecting a merge area are displayed. In the next section the components of the control system are displayed. 


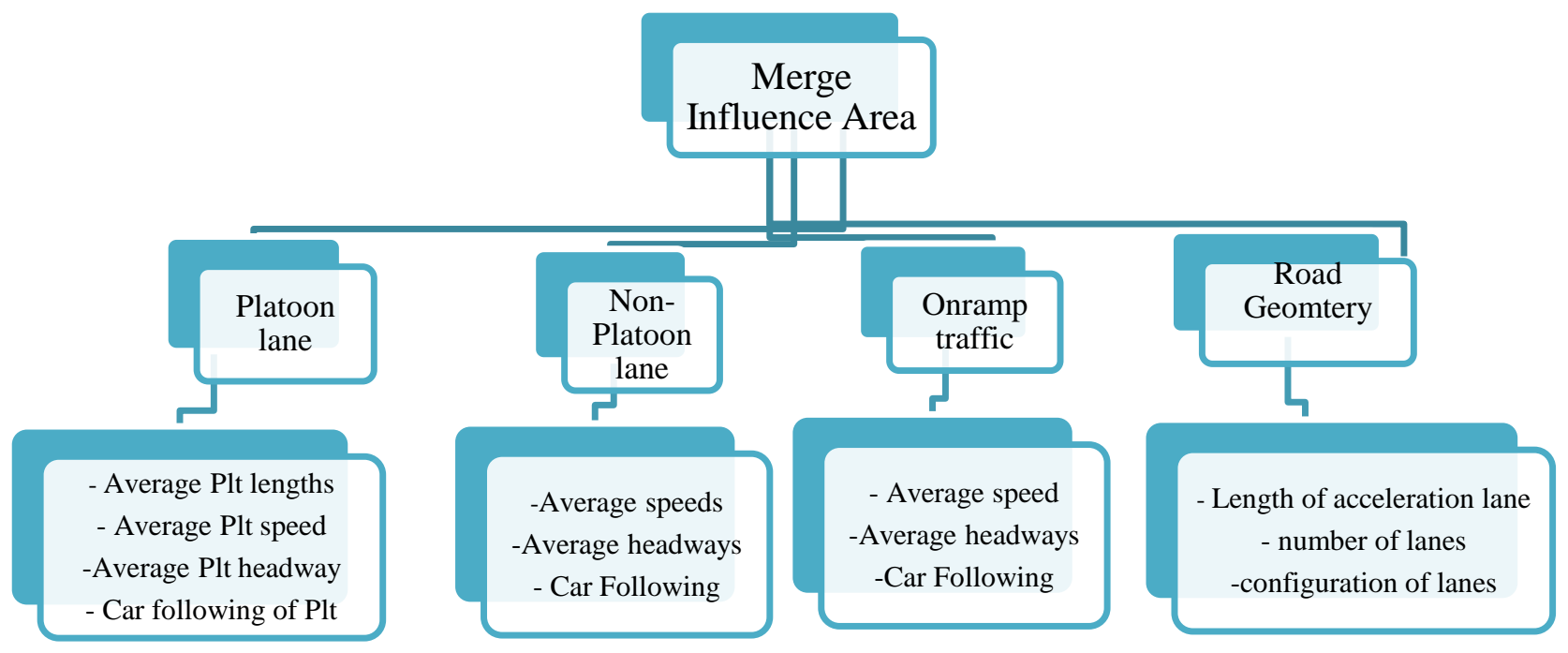

Figure 6 The model components affecting the merge influence area

\subsubsection{Control Model Attributes}

The control algorithm is designed to direct the onramp merging cars onto the rightmost lane (lane 1) of the highway. The control algorithm follows the defined conditions based on a rule-based model for enabling the required control actions to take place. Figure 7 displays the different components involved in a merge control. The control action thresholds determine the type and intensity of the action which will be implemented by the control actors. 


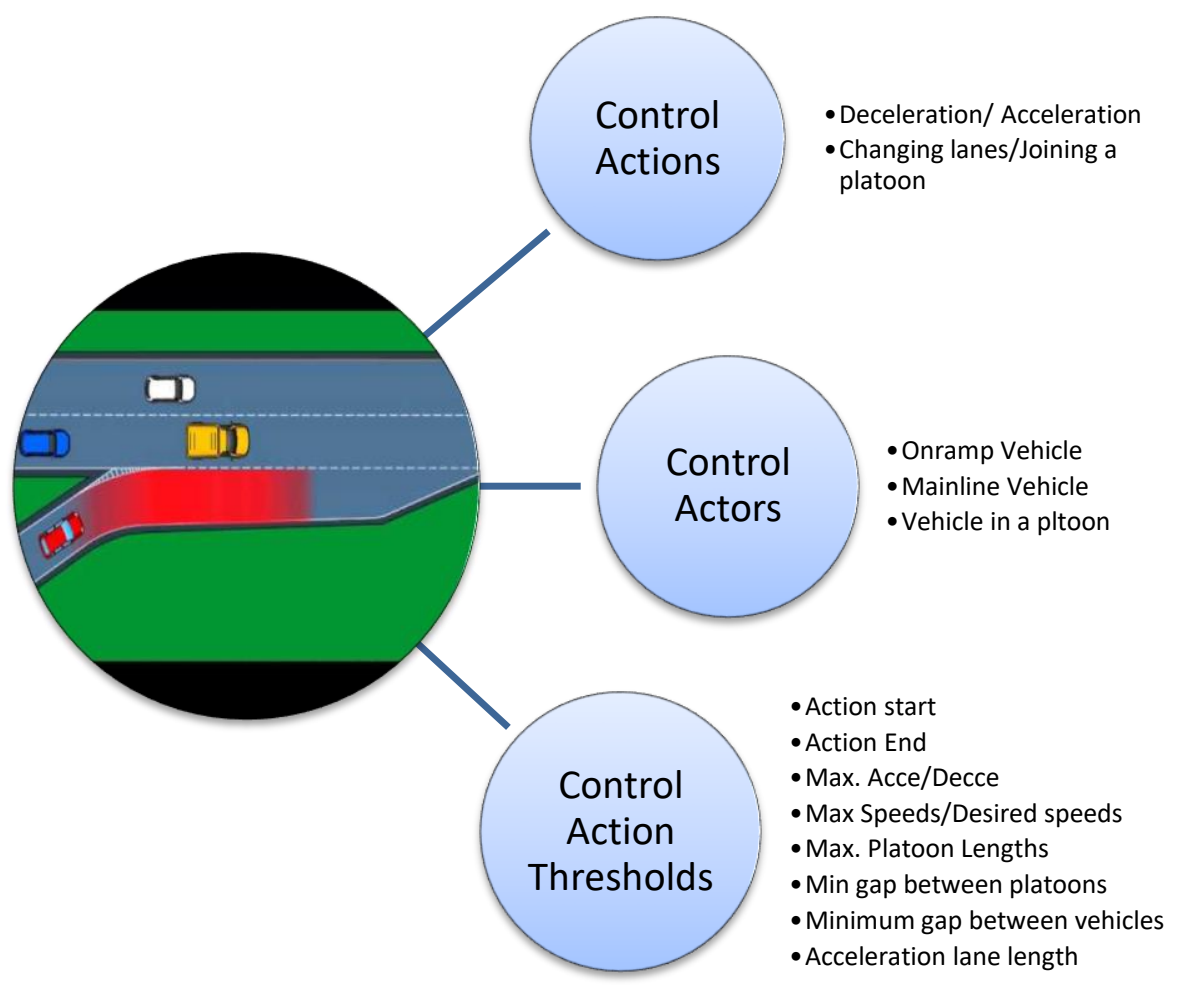

Figure 7 Parameters of the control section in the model

The control action thresholds displayed in each have a certain level of influence on the merge area. As an example, larger headways between vehicles on non-platoon lane will make more possible merging gaps for the onramp vehicles. At the same time larger platoon lane throughputs means it is less possible for vehicles to join platoons and generate gaps for onramp vehicles. The control action thresholds will be further discussed in this chapter.

\section{Technological Requirements of the model}

The merge model in this study requires the operation of specific technologies. In the list below, the requirements for the functionality of the model are explained:

- Fast and reliable connectivity between the vehicles and the central controller such as the 5G communication for relaying required information between vehicles and infrastructure

- Connectivity between vehicles with other surrounding vehicles

To enhance safety between vehicles in the same vicinity 
- Traffic detection of onramp and mainline vehicles for the controller to plan the necessary actions

- Processing power of the central controller in order to make decisions and send the required information

- Driver assistance systems (Collision avoidance, Adaptive cruise control, autopilot in the lane change mode)

To summarize the sections above, the model operation will follow the steps listed below for the completion of every merge analysis.

- Simulation of traffic in a multilane highway

- Merging vehicle traffic

- Processing and decision-making section

- Implementing the control action

- Analysis of the state-action results

The model inputs can be classified in two groups, external inputs such as traffic demand and onramp geometry, and internal inputs such as control parameter factors and car following behavior. The desired outputs of the model can be set by the user and include, throughput, average travel time etc.

\subsubsection{Model specifications}

The mainline traffic is comprised of platoon lanes and a non-platoon lane. The non-platoon lane is associated with vehicles which want to leave the highway, or which cannot or do not desire to be traveling in the platoons. Additionally, the highway segment is assumed to be equipped with communications between the mainline CAVs, onramp CAVs, and the infrastructure. Therefore, the highway is a connected highway with communication between the components of the system. The communications in this merging point are Vehicle-Infrastructure (V2I), Vehicle to vehicle (V2V), and Infrastructure to Vehicle (I2V). Vehicles traveling in the platoons are under automated driving and vehicles in the non-platoon lanes are connected and can be converted to automated vehicles when they join the platoon. 
The range of communications in this realm are as long as the segment of the highway under study, upstream of the merging location. The merging onramp has an acceleration lane adjacent to the highway segment as can be seen in Figure 2.

The volumes in this geometry are, $q_{\text {ramp }}, q_{\text {merge }}, q_{(1)}, q_{\text {lane } 1}$, which are onramp demand, onramp merging volume, throughput downstream the onramp and volume of mainline(s) before the onramp, respectively.

The vehicles communicate for the execution of a merge action, whilst the type of action is determined by the central controller which monitors the traffic status upstream the merge area. Whenever an action is necessary for the merge, the central controller sends the information for the type of action to the target vehicle and the target vehicle completes the action while it is in a constant connectivity with the merging vehicle.

The car following of the non-platoon lanes in the model is the IDM+ car following model which is an extension of the IDM car following. The platoon lane has a simplified CACC car following. The CACC will make platoons to accelerate or decelerate according to the lead platoon. For the concept simulated in this study, the connected Highway (connection between all vehicles), dedicated lanes for platoons of connected-automated vehicles, merging from an onramp are the primary parts of the simulation. Additionally, the geometric features influencing the merge area performance are: Length of acceleration lane, length of upstream traffic observed for action determination.

\subsubsection{Lane 1 traffic flow}

The merging vehicles will merge onto lane 1 of the multilane highway. Lane 1 traffic will be simulating a non-platoon lane with connected vehicles. The specifications used in the model for lane 1 are listed below:

- IDM+ car following

- Cooperative merge enabled by deceleration to create gaps for merging vehicles

Lane 1 of the model will be operating as a non-platoon lane, since many vehicles intending to leave the highway from an offramp will not be traveling on the platoon lane. Additionally, it can be assumed that certain vehicles traveling a short distance on the highway will not enter the platoon lane. Furthermore, certain vehicles on the highway may not desire to be traveling within a platoon and thus 
the rightmost lane will be a non-platoon lane. Figure 8 displays the significant parameters in the merge area traffic model of this study.

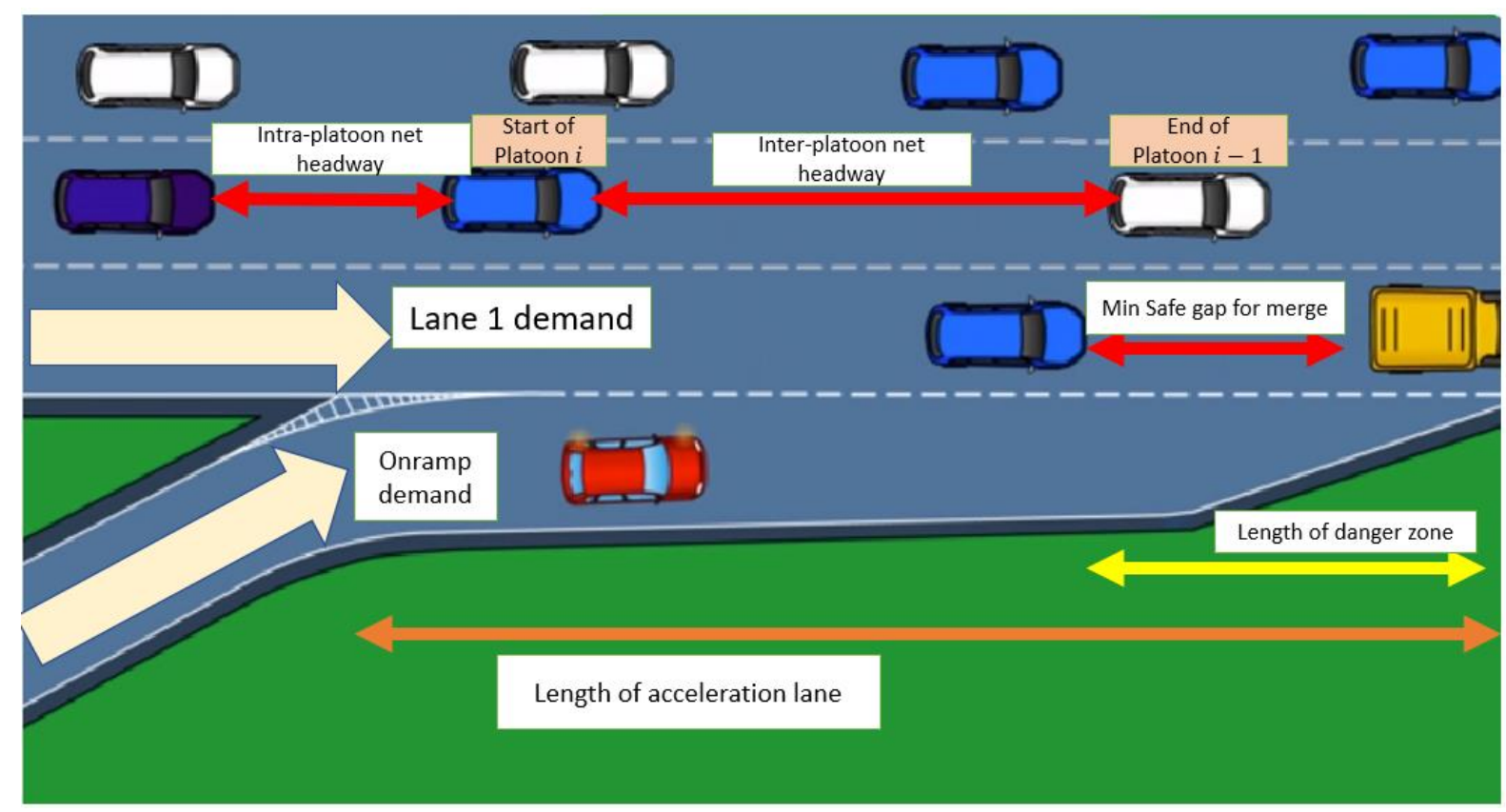

Figure 8 The merge model area and relevant model parameters

The car following model for vehicles in lane 1 and the onramp merge is the IDM+ car following model which is a modified version of the IDM model. This car following has the Equation 1 and 2 for the acceleration rates:

$$
\begin{array}{ll}
\frac{d v}{d t}=a \cdot\left[1-\left(\frac{v}{v_{0}}\right)^{4}-\left(\frac{s^{*}(v, \Delta v)}{s}\right)^{2}\right] & \text { Equation 1 } \\
\text { With, } s^{*}(v, \Delta v)=s_{0}+v T+\frac{v \Delta v}{2 \sqrt{a b}} & \text { Equation 2 }
\end{array}
$$

Where $a$ is the comfortable acceleration, $v$ is the current speed, $v_{0}$ is the desired speed, $s_{0}$ is the minimum headway (at standstill), $T$ is the desired time headway, $\Delta v$ is the speed difference with the leader, $s$ is the current distance headway and $b$ is the comfortable deceleration. IDM+ has a capacity of approximately $2000 \frac{\mathrm{veh}}{\mathrm{hr}}$. The rate of each parameter mentioned above for the model in this study are listed below: 
- $v=\frac{35 m}{s}$

- $T=1.6 s$

- $s_{0}=2 m$

- $a=\frac{1 m}{s^{2}}, b=-2 \frac{m}{s^{2}}$

In order to increase this capacity of lane 1, considering the advent of Advanced Driver Assistance Systems (ADAS), adaptive cruise control and collision avoidance systems for all of the connected cars, the additional controls below were added to this car following model for the onramp and lane 1 vehicles. The additional controls were achieved through numerous trial and error simulation runs for testing the effect of each control and obtaining the combined effects of both controls.

The additional controls will enable cars to follow with shorter headways due to the faster reaction time and additional safety that they provide.

1. If the gap between a vehicle and the front vehicle is larger than $32 \mathrm{~m}$, it will accelerate as free-flow condition (Equation 3):

$$
x_{i-1}-x_{i}>32 m \Rightarrow a_{i}=a_{\text {freeflow }} \quad \text { Equation } 3
$$

2. If a vehicle has a gap less than $15 \mathrm{~m}$ to the front vehicle, it will adopt the speed of the front vehicle minus a safety rate $(\mathcal{E})$ (Equation 4$)$ :

$$
x_{i-1}-x_{i}<15 m \Rightarrow v_{i}=\left(v_{i-1}\right)-\varepsilon \quad \text { Equation } 4
$$

\subsubsection{Platoon lane traffic flow}

The platoon lane will have platoons arriving from the upstream with different formations. Each platoon has several attributes. They are:

- Number of vehicles in the platoon, $\mathrm{N}$

- Intra-platoon headway, i

- Inter-platoon headway, h

- $\quad$ Speed of platoon $(\mathrm{V})$ 
For simplicity, sometimes researchers consider platoons of CAVs as a homogenous entity with a constant intra-platoon headway. The inter-platoon headway and platoon lengths can be a varying parameter due to the stochastic and random nature of traffic. It is expected that in a fully automated highway with $100 \%$ CAVs, due to the existence of onramps and offramps, different destinations in the network etc., the platoons arriving from upstream may not have the same number of vehicles within them.

A lane which has platoons of CAVs can be viewed both from a microscopic and macroscopic perspective. In the microscopic vision, the longitudinal movements, speeds, and traffic behavior passing a point can be under investigation. From a macroscopic perspective the effects of the platoons on throughputs, average speeds etc. are under attention.

The maximum number of vehicles which would pass a fixed point by platoons of CAVs can be calculated by the Equation 5:

$$
\text { Throughput (Capacity) }=3600 * \frac{v \cdot N}{N \cdot L+(N-1) i+h} \quad \text { Equation } 5
$$

In which $\mathrm{L}$ is the (average) length of the vehicles in the platoon, while $\mathrm{h}$ and $\mathrm{i}$ are inter-platoon and intra-platoon spacing respectively. Inter-platoon headway is the net time headway between consecutive platoons while intra-platoon headway is the net time headway between consecutive vehicles within one platoon.

Fundamental traffic engineering and the equation 5 show that higher platoon speeds and shorter inter/intra platoon headways yield higher throughputs. Furthermore, higher number of vehicles in a platoon also leads to higher throughputs. The relation between speed and throughput of a platoon lane is linear, but the relation between number of vehicles in a platoon and platoon lane throughput is nonlinear. Table 2 displays the effect of attributes in a platoon lane. 
Table 2 Effect of platoon parameters in platoon lane throughput

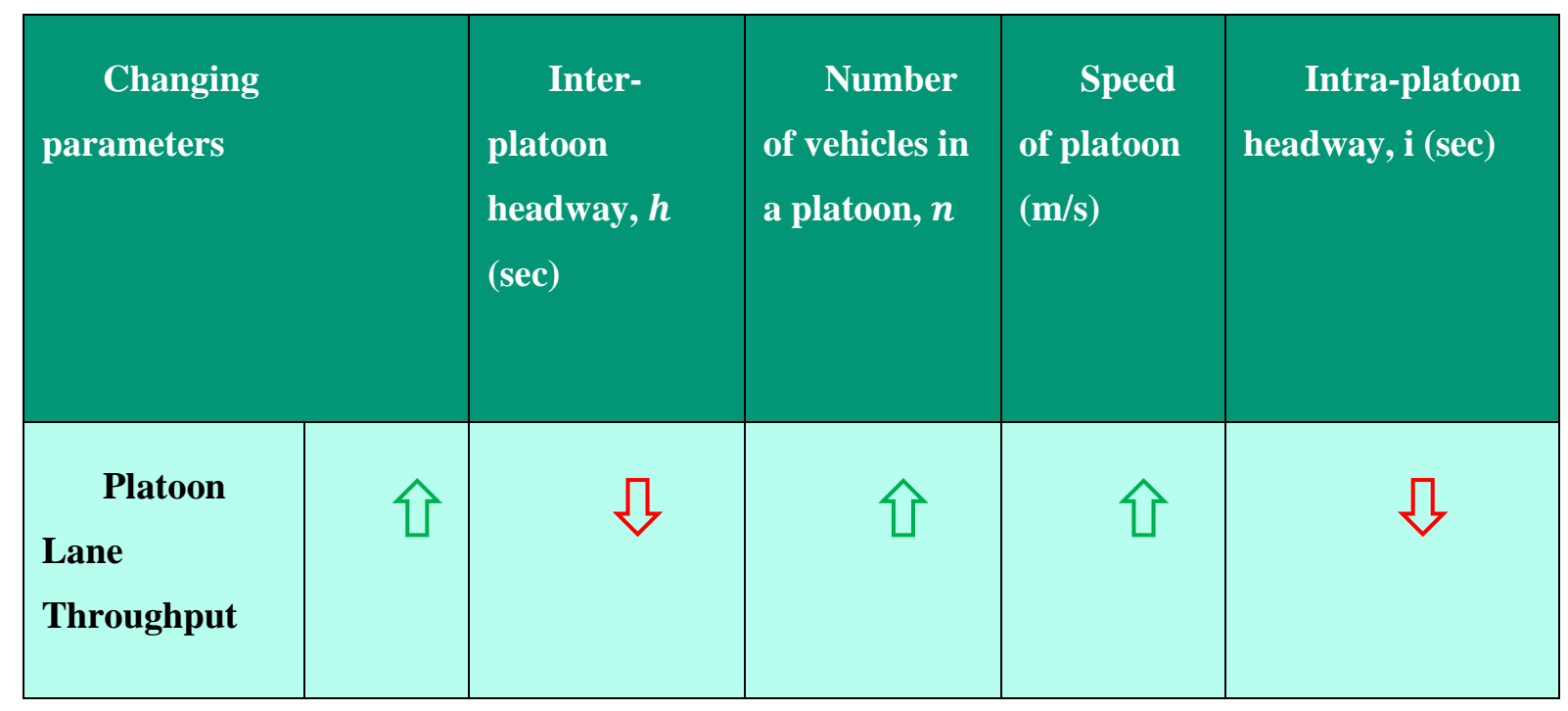

\section{Capacity of platoon lane}

The capacity of the platoon lane is obtained by the maximum possible length of platoons and minimum inter-platoon and intra-platoon headways. Numerous tests with different platoon features are implemented in the world. Currently in the literature, previous research does not provide the specific length of the platoons which will be in the traffic of the highways. Therefore, it is not possible to determine a capacity for platoon lanes at this point of time.

By utilizing the equation 5, the results in Figure 9 to 11 display the increasing throughput of the platoon lane via increasing the platoon length and speed. 


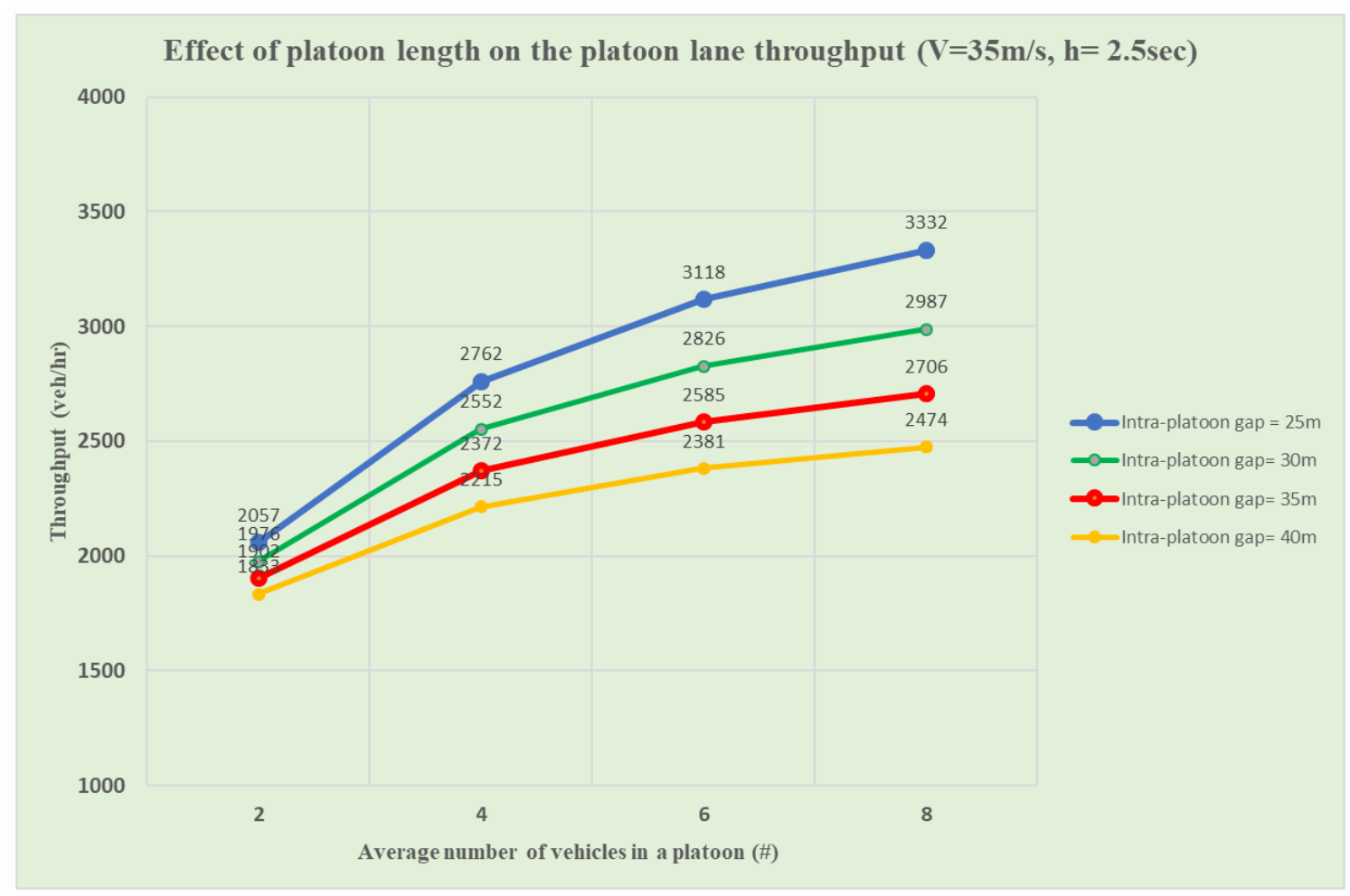

Figure 9 Effect of platoon length on platoon lane throughput

\section{Effect of platoon speed on the throughput of the platoon lane}

In this test, the effect of platoon speeds on the throughput of the platoon lane were tested with fixed rates for other parameters of the platoon lane. The inter-platoon gaps in this test were set at 80 meters which with the average speeds for the platoons, corresponded to the inter-platoon headway of 2.67 seconds. This rate of inter-platoon headway range was assumed in this study for providing the safe gaps between consecutive platoons after revision of similar research. 


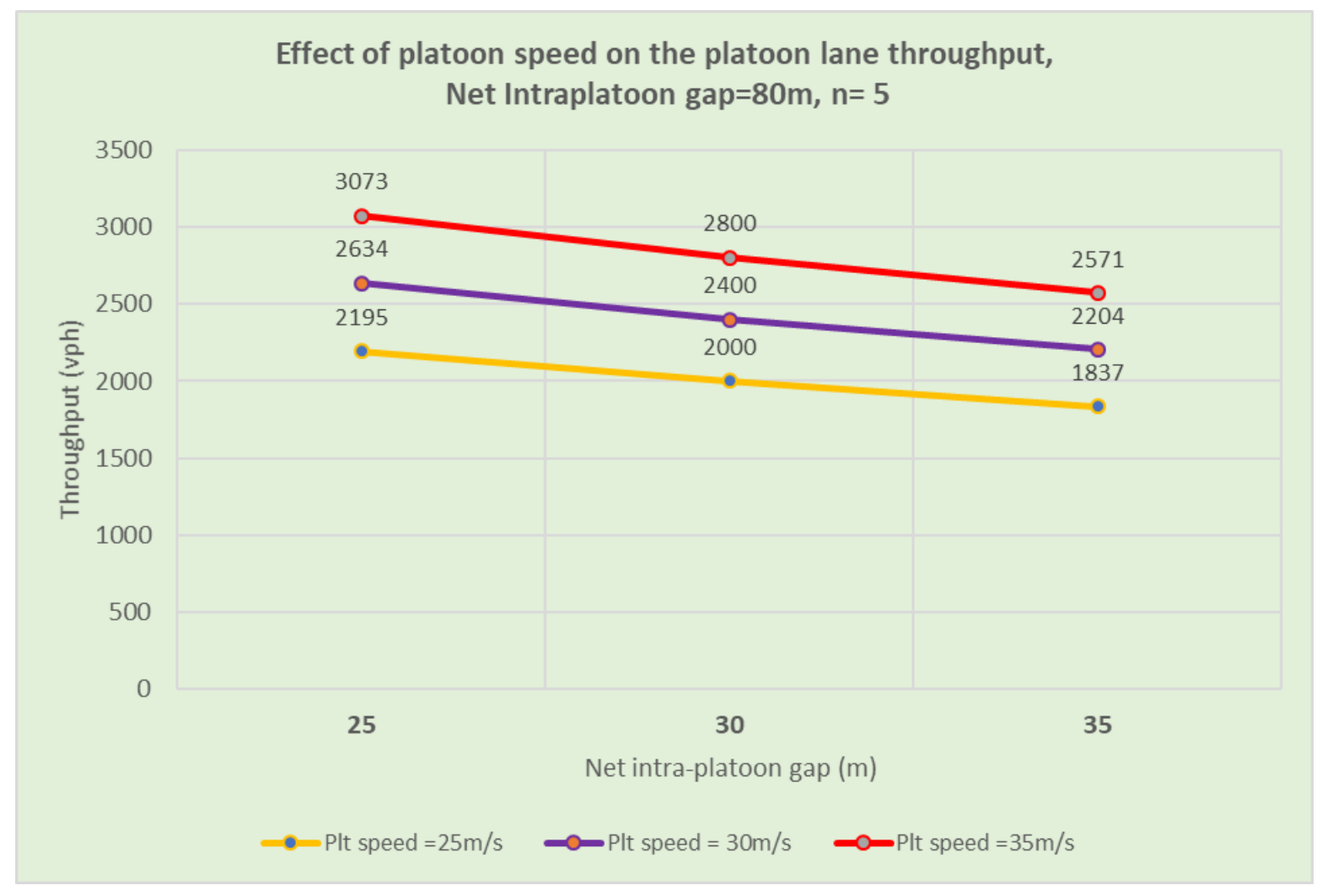

Figure 10 Effect of platoon speed on the platoon lane throughput

\section{Effect of inter-platoon gap on the throughput of the platoon lane}

In this test, the effect of inter-platoon headway was tested while maintaining constant amounts for other parameters of the platoon lane. The intra-platoon headways were set to 25 meters $(0.7 \mathrm{sec})$ which in this study is considered as the minimum gap between vehicles in a platoon. 


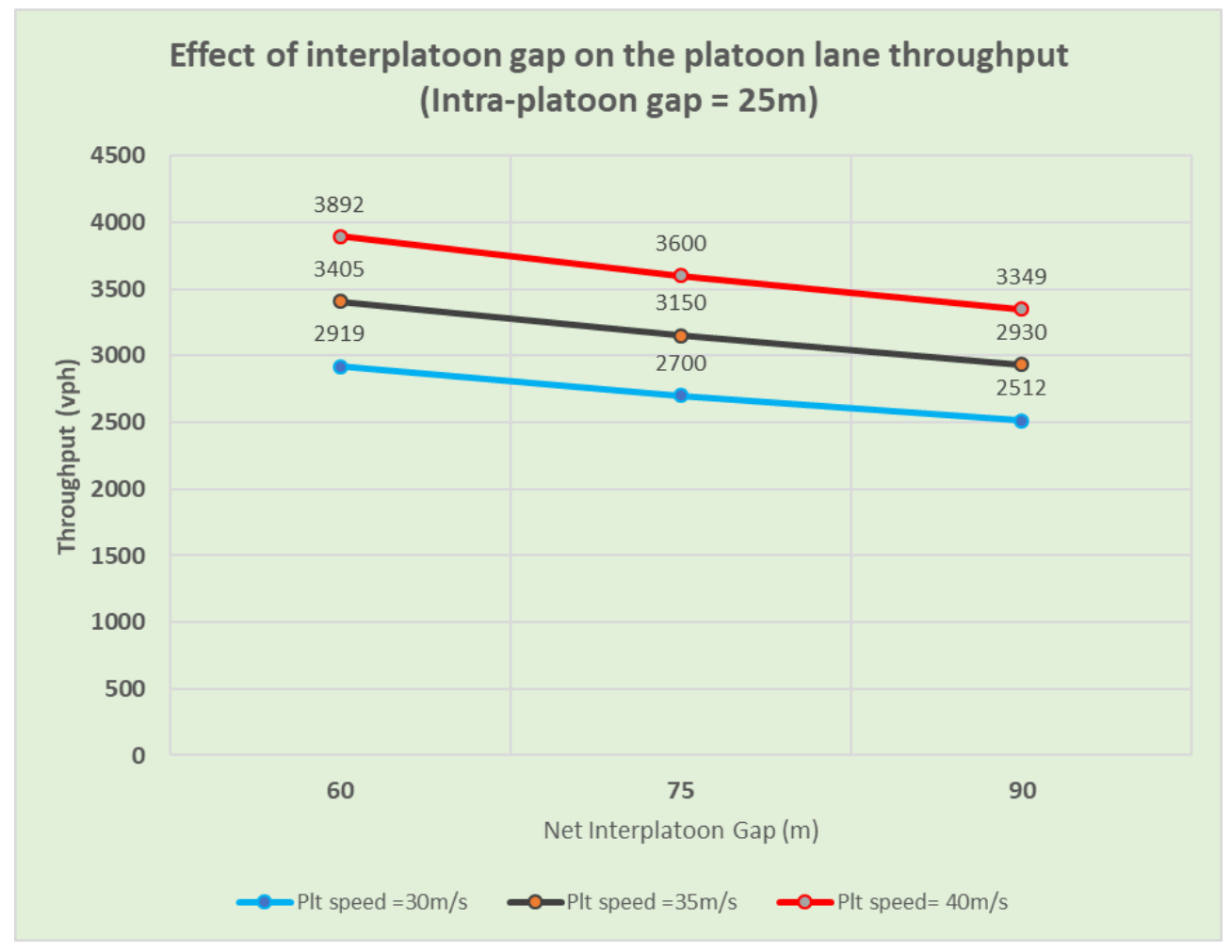

\section{Figure 11 Effect of inter-platoon gap on the throughput of the platoon lane}

As can be seen in the figures 9 to 11, the platoon length has a non-linear relation with the throughput of the platoon lane. Increasing the speed of the vehicles in platoons and reducing the interplatoon and intra-platoon headways have a direct linear relationship with the throughput of the platoon lane.

\subsection{Action Specifications}

\subsubsection{Merge with Action 1}

Action 1 utilizes deceleration on lane 1 or onramp to create gaps for merging vehicles. The merge model in this study is according to the basis of preventing actions (for gap creation) on the mainlines unless necessary. Thus, this action will only take place if the merging vehicle is on the danger zone of the acceleration lane. 
The aim of this merge type is creating a merging control action with required actions of merging and mainline vehicles using the principle of Model Predictive Control in controlling the actions of the merging vehicle. In other words, in the situation that a safe gap is unavailable for merge, the merging vehicle adapts its driving to the largest gap feasible for joining in every timestep while projecting the largest gap of the next timestep. Besides that, the merging vehicle is also ready to join a gap if at any timestep the gap is safe according to the MOBIL lane change model (reference). This enables a dynamic framework for merging while preventing a merge action until its necessary.

\section{Danger zone on the acceleration lane}

The term "danger zone on acceleration lane" in this research refers to a specific length before the end of the acceleration lane which the merging vehicle must merge in that zone or otherwise stop. In this research the possibility of merging vehicles having to come to a full stop is not investigated and therefore the model has contingent actions occurring to assist the merging vehicle before the vehicle approaches the end of the acceleration lane. The danger zone is the segment marked as visual clear in Figure 2.

\section{MOBIL Lane Change Model}

The MOBIL (Minimized Lane Change Deceleration Induced by Lane Change) is classified as one of the lane changes with courtesy models. The MOBIL lane change model is comprised of a component called the politeness factor indicating the degree of consideration of other drivers if there are no safety restraints (Kesting et al., 2007).

The MOBIL lane change model defines the boundary conditions for a lane change to occur. The model is formulated for different car following models. Figure 12 displays the safe gap to merge between two vehicles according to speeds of the vehicles in the merge process. Equation 6 displays the minimum safe gap recommended by MOBIL model for the IDM model.

$$
\hat{s}_{\hat{f}}>s_{\text {safe }}^{I D M}\left(v_{\hat{f}}, v_{\alpha}\right)=\frac{s^{*}\left(v_{\hat{f}}, v_{\hat{f}}-v_{\alpha}\right)}{\sqrt{\frac{1}{a}\left(a_{\text {free }}\left(v_{\hat{f}}\right)+b_{\text {safe }}\right)}} \quad \text { Equation } 6
$$

In which $b_{\text {safe }}$ is the desired deceleration rate, $a_{\text {free }}$ is the freeflow acceleration, $v_{\hat{f}}$ is the speed of the follower and $s^{*}$ is the dynamical desired IDM gap. $v_{\alpha}$ is the speed of the lane changing vehicle. 


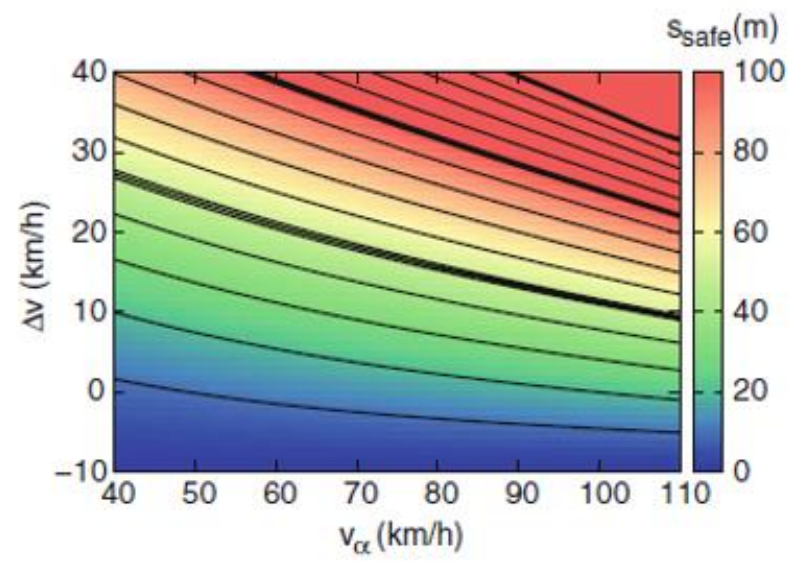

Figure 12 The required safe gap for merge according to MOBIL model

As can be seen in Figure 12, with two vehicles in two adjacent lanes driving in speeds higher than $30 \frac{\mathrm{m}}{\mathrm{s}}(108 \mathrm{~km} / \mathrm{h})$ and a speed difference of $5 \frac{\mathrm{m}}{\mathrm{s}}(18 \mathrm{~km} / \mathrm{h})$, the minimum safe gap required for merge from the MOBIL model is approximately 35 meters. In the model of this study, the vehicles in the mainlines are driving at speeds of $35 \frac{\mathrm{m}}{\mathrm{s}}$ and have speed differences less than $5 \frac{\mathrm{m}}{\mathrm{s}}$. For additional safety for the vehicles, the minimum safe gap for merge considered was 45 meters.

The basic steps in merge with action 1 (deceleration for gap creation) is according to the basics listed below:

- From the timestep $t_{1}$, that the merging vehicle enters the acceleration lane, a potential merging space in its surrounding - which it can join for merging - is defined. This space corresponds to the distance relative to \pm 1 sec headway which in this research accounts for safety and average headway between vehicles in lane 1 as a non-platoon lane. This space dynamically changes with the movement of the merging vehicle and is also observed by the local traffic controller. Figure 13 displays the potential merging space within the acceleration lane depicted above. 


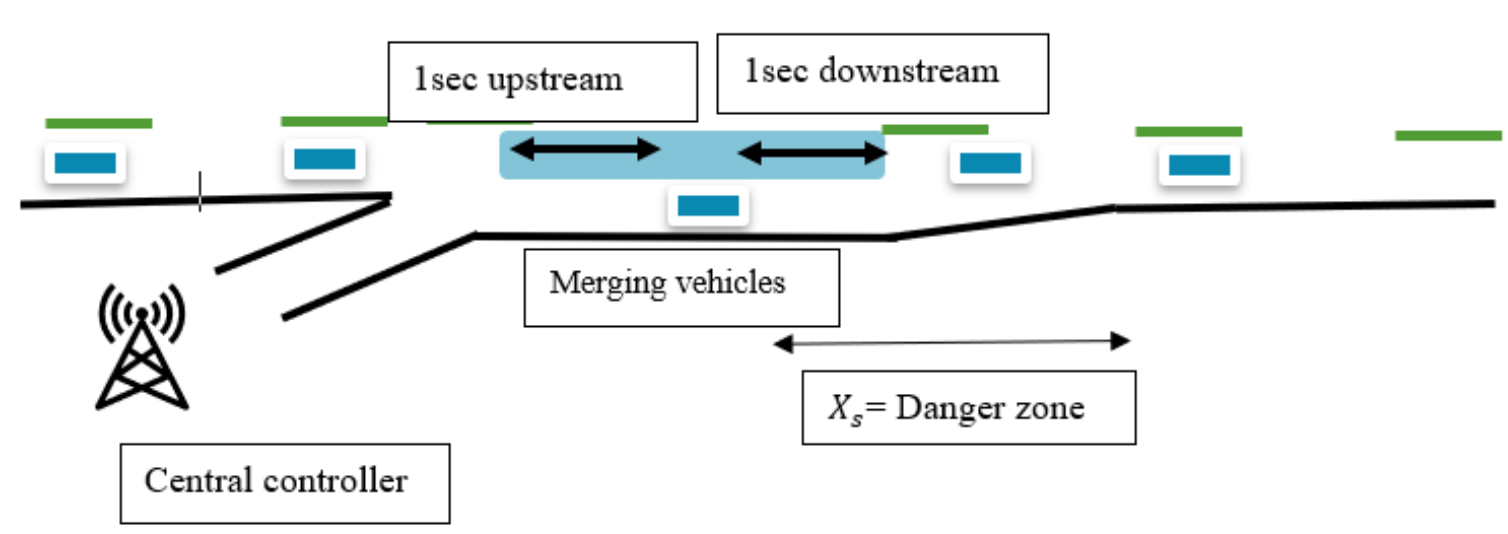

Figure 13 The potential space for merging

- If within the moving space of the merging vehicle there is a safe gap large enough according to the lane change model of MOBIL at that timestep, the vehicle will merge onto that gap.

- If a suitable gap for merging in timestep $t_{1}$ is not available, the merging vehicle estimates the suitable upcoming gaps available in the next timestep, $t_{2}-$ while traveling on the acceleration lane- this step in this research is called the Gap Projection. If the gaps at the next timestep would be safe to merge, the onramp vehicle modifies its movement to adapt to the merge gap in range available in the next timestep. This step is called the gap adaption.

- If the merging vehicle cannot merge within a safe distance from the end of acceleration lane, a cooperative merge will take place with action one requirement transferred from the central controller, therefore disturbance of the lane 1 traffic won't happen unless necessary. In other words, the occurrence of action 1 is the last resort taking place when the merging vehicle is on the danger zone. 
The steps of merge type 1 are displayed in

. The parameters used in the Figure 13 and Table 3 are:

- Next Max Gap Detection: Distance is the distance observed for identifying the gaps in the next timestep

- MinSafeGap: the minimum safe gap required for merge according to MOBIL model.

- MaxGap: the maximum identified gap in range

- Detection distance is the distance observed by the merging vehicle.

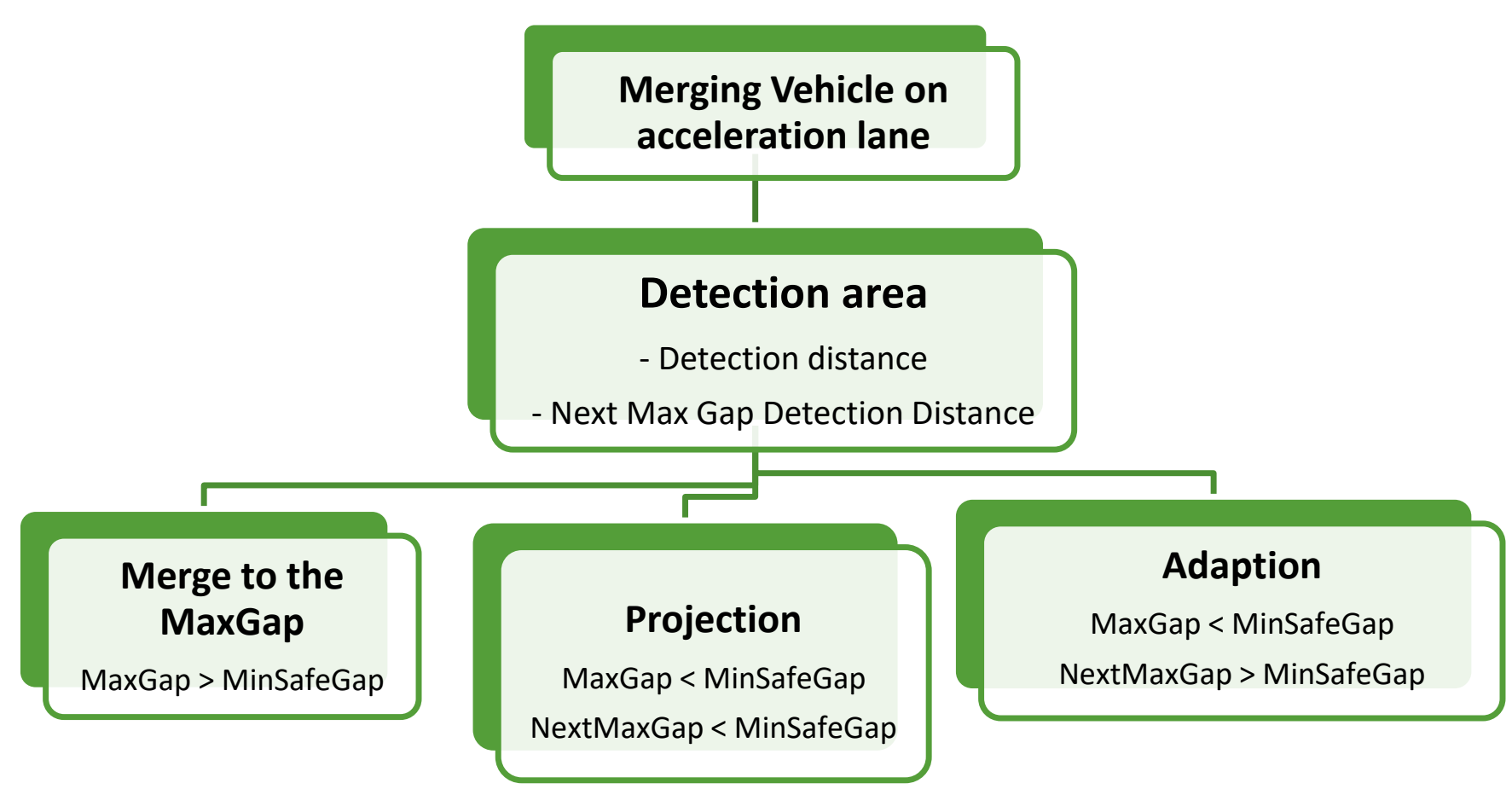

Figure 14 Merging scenarios with action 1 
Table 3 Merge conditions for onramp vehicles on the acceleration lane

\begin{tabular}{|c|c|c|}
\hline \multirow[t]{2}{*}{ Merge conditions } & \multicolumn{2}{|c|}{ Merging requirements } \\
\hline & Out of danger zone & Inside danger zone \\
\hline $\begin{array}{l}\text { State } 1 \\
\text { MaxGap > Gap required }\end{array}$ & Merge to MaxGap & - \\
\hline $\begin{array}{l}\text { State } 2 \\
\text { MaxGap < Gap required } \\
\text { NextGap > Gap required }\end{array}$ & Adaption to NextMaxGap & $\begin{array}{l}\text { Action required for merging } \\
\text { to max gap }\end{array}$ \\
\hline $\begin{array}{l}\text { State } 3 \\
\text { MaxGap < Gap required } \\
\text { NextGap < Gap required }\end{array}$ & Continued Projection of NextGap & $\begin{array}{l}\text { Action required for merging } \\
\text { to max gap }\end{array}$ \\
\hline
\end{tabular}

In a Model Predictive Control, in every timestep, the controller measures the efficient parameters for the upcoming stages of the system. At every timestep, the system proceeds with the efficient parameters calculated for that next timestep. In this research the application of this control is effective for determining the next action for every merging vehicle. The controller decides the type of action the merging vehicle must pursue to deliver a merge in every timestep that the vehicle is on the acceleration lane. The controller's decision is based upon the ground events and the actions required for the merge. The actions which the controller orders for the merging vehicle are explained in the next section.

\section{Steps in deceleration to form a larger gap}

Figure 15 schematic displays the notation used for the vehicles which are in the merge process. In figure 15, vehicle $\alpha$, (in red) is the merging vehicle, vehicle $f$ is the expected follower of the merging vehicle. Vehicle $l$ is the expected leader of the merging vehicle. When $\alpha$ requires an action, initially, the required gap is calculated by Equation 7:

$$
S_{\text {req }}=S_{\text {safe }}-S_{\hat{f}} \quad \text { Equation } 7
$$


In which $S_{\text {safe }}$ is the minimum safe gap required for merging according to lane change model with relaxation by MOBIL model and $S_{\hat{f}}$ is the current gap available for merging. According to $S_{\text {req }}$ and the maximum accepted deceleration, $b$ the time required for deceleration $t_{d e c}$ is calculated according to Newtonian physics which can is shown on Equation 8, considering the onramp and mainline have adapted speeds.

$$
t_{\text {dec }}=\sqrt{\frac{2 \cdot S_{\text {req }}}{b}} \quad \text { Equation } 8
$$

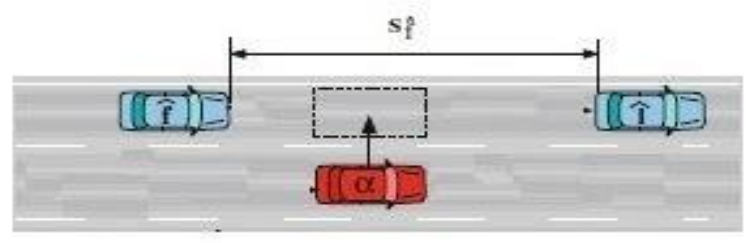

Figure 15 The merging vehicle and the adjacent lane

The algorithm (pseudocode) for the deceleration action applied in the simulation model is listed below:

Follower vehicle is detected based on the largest gap within range on lane 1

FOR follower vehicle $i$ on the non-platoon lane

Decelerate for minimum $t$ seconds with deceleration $=b$ until the updated gap is suitable for merging

Merge completed

END FOR

Figure 16 displays the flowchart of the action 1 steps. In the flowchart the minimum safe gap is displayed as S(MOBIL) and the estimated gap in the next timestep is NextMaxGap. 


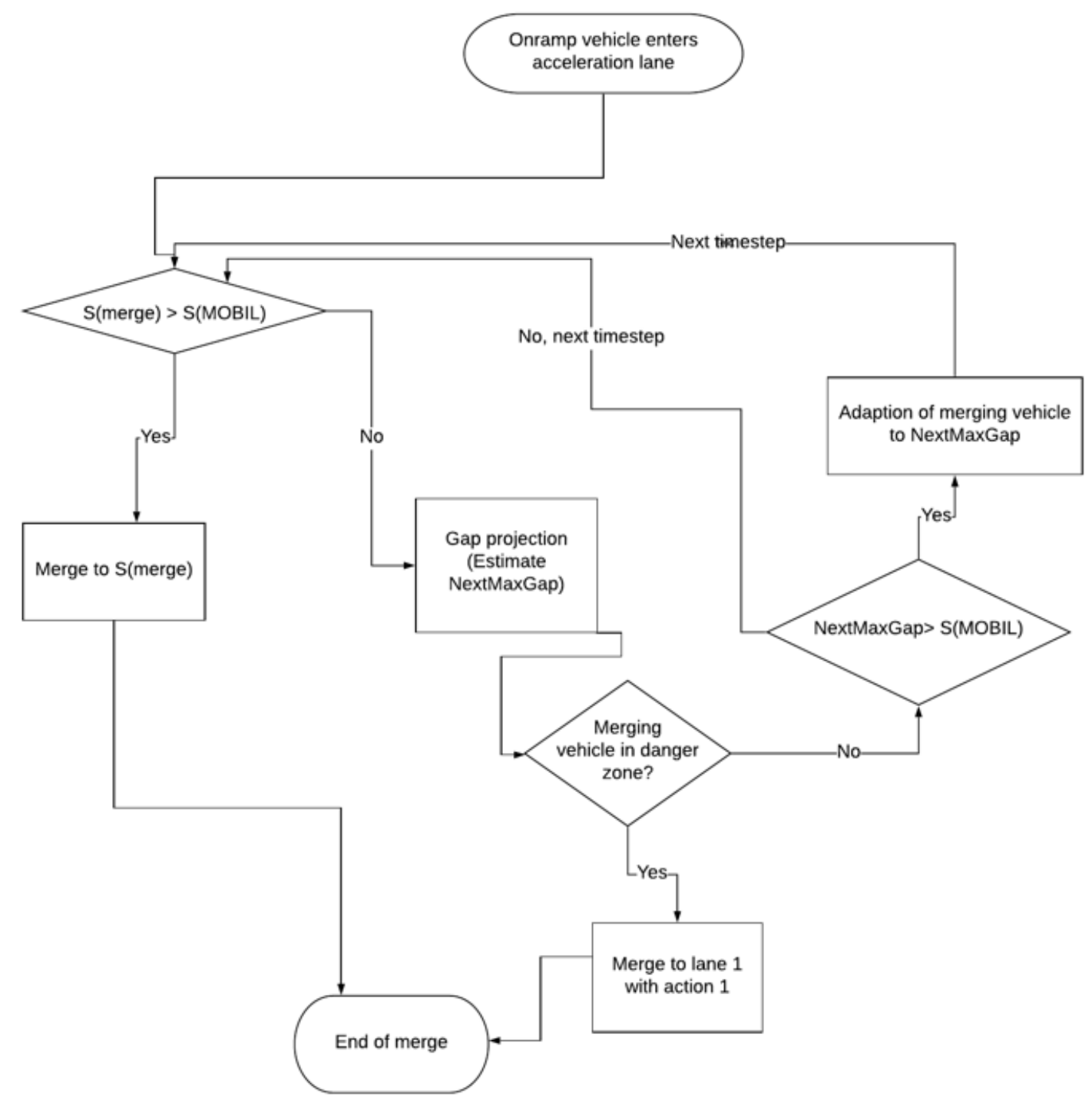

Figure 16 Steps of merge type 1

\section{Gap Projection}

Projection of the next timestep by the merging vehicle is measured via the speed, vehicle position, and current gap between vehicles besides an error which is natural for any system. Equation 9 displays how the gap in the next timestep is measured from the gap in the current timestep.

$$
\operatorname{Gap}_{t+1}=\operatorname{Gap}_{t}+\left[\Delta v=\left(v_{l}-v_{f}\right)\right]+\varepsilon \quad \text { Equation } 9
$$


In which $\operatorname{Gap}_{t+1}$ is the gap between vehicles in time $t+1$, while $\operatorname{Gap}_{t}$ is the gap between vehicles in time $t$. The speed of the leading vehicle is $v_{l}$ and the speed of the following vehicle is $v_{f}$. The error in the measurement is displayed by $\varepsilon$.

Assumptions: The onramp vehicle seeks to find a gap in lane 1 via predicting the lane 1 vehicle positions in the next timestep. This prediction is based on the current speeds and gaps between vehicles. The prediction is completed every timestep for the next 1 second. This 1 second is due to the limited time of merging for onramp vehicles. The parameter $\varepsilon$ accounts for minor system errors and was selected from a uniform range between $(0,1)$.

\section{Gap Adaption}

When the merging gap is projected for the next timestep, the gap adaption is the action by the merging vehicle which aims at reducing the distance of the merging vehicle to the merging gap identified for the next timestep. After a merging vehicle has identified a potential merging gap through the projections or through detection, it will pursue the necessary actions in order to adapt its position for potential merging onto the specified gap. It must be noted that in the presence of a more suitable gap in the next timestep, the vehicle's adaption operations will be altered. Therefore, the actions corresponding to an adaption/projection or merging are updated in every timestep. The pseudocode below depicts the required steps for the gap projection and gap adaption by merging vehicles.

Check lane 1 gaps at the start of speeding lane $(x=200)$

IF (an available gap is suitable for merge)

Merge onto the lane 1

ELSE IF (Safe gap is not available)

Project the next vehicle positions and detect largest gap in the next timestep if merging vehicle is closing in on the acceleration lane Cooperative action for merging takes place based on mainline densities Onramp merging vehicle merges,

else

if safe gap is available for merge in next timestep,

Onramp vehicle adapts movement to approach the suitable gap

else project the next vehicle positions and detect largest gap in the next timestep end if end if

END IF

End Merge action 
Whenever there is no sufficient safe gap available for merging and the merging vehicle is closing up on the acceleration lane - inside the danger zone-, a merge action on lane 1 via the guidance of the central controller takes place. The merge action 1 will insure the safe gap for merging through required deceleration on lane 1 .

\section{Summary of Action 1 and Thresholds:}

When a merging vehicle is on the acceleration lane, it controls the gaps feasible to join (gaps within distance corresponding to the $T_{\text {merge }}{ }^{3}$ limits on the rightmost lane). The estimate of the latest merging time of the onramp vehicle is determined with the Equation 10:

$$
t_{\text {onramp }}=\frac{x_{\text {accel }}}{v_{\text {Rampleh }}} \quad \text { Equation } 10
$$

If the safe gap is available, the vehicle merges to that gap in the next timestep, otherwise the vehicle estimates the gaps in the next timesteps which are in a $T_{\text {proj }}$ (projection headway) headway range upstream or downstream of its current position (the $T_{\text {proj }}$ differs based on average speeds and headways of the traffic). The $T_{\text {proj }}$ is larger than $T_{\text {merge }}$ as the merging vehicle seeks to find safe gaps in farther distances. If the estimates reveal a safe gap, the vehicle adapts its acceleration such that it approaches the gap faster. In this study $T_{\text {proj }}$ was set to 1.5 seconds and $T_{\text {merge }}$ was set to 1 second as feasible rates for merging.

In the next timestep, in the instance of no safe gap available for merge, the vehicle projects gaps again, unless it has passed a safety margin-referred as the danger zone in this research- which in that case, the merging vehicle would require an action from the mainline vehicles. The vehicle on lane 1 with the least relative longitudinal difference with the onramp CAV executes the action for creating a

\footnotetext{
${ }^{3} T_{\text {merge }}$ is the headway that corresponds to the distance in front or behind the merging vehicle which the
} merging vehicle has the possibility to join in terms of safety and vehicular dynamics constraints. In this research this number was selected at 1 second. 
gap. In this research after the exhaustive search for an acceptable range feasible for merging, the value of 1 and 1.6 seconds were selected for $T_{m e r g e}$ and $T_{\text {proj }}$ respectively.

The basis for defining the danger zone is the distance which provides enough time for any safe gap to be created. Therefore, the absolute length of the danger zone, $L_{d z}$, should be larger than minimum time required for gap creation by the mainline vehicles $T_{\text {action }}$.

$$
L_{d z}>\min \left(T_{\text {action }}\right) \quad \text { Equation } 11
$$

Regarding the condition mentioned in equation 4 and the physical limitations for a merge requiring an action on the mainlines, a minimum of $100 \mathrm{~m}$ is required for the design of the danger zone. The $100 \mathrm{~m}$ is associated with approximately 3 seconds of movement and 1 second for lane change to yield a safe merging through an action.

The propagating effect of action 1 depends on the factors below:

- The gap between the decelerating vehicle and it's following vehicle

- The deceleration rate which is dependent on the gap between the decelerating vehicle and its front vehicle

The additional controls for the IDM+ car following model in this study were tested to provide a positive effect in reducing the perturbations caused by action 1 .

\subsubsection{Merge with action 2}

In action 2, the lane change movement will be utilized to create gap for the merging vehicle. Selected vehicle on lane 1 will join a platoon in lane 2 to create a gap for the merging vehicle. Unlike action1, the merging vehicle is detected before entering the acceleration lane. The onramp vehicle is detected by roadside sensor and the vehicle's information is relayed to the central controller. The central controller detects the gaps feasible for merging on lane 1. If a suitable gap for merge is not available, a specific target vehicle is selected to move lane from lane 1 to lane 2 . Vehicles respond to the relayed message (from the central controller) based on a defined compliance rate. The compliance rate is the probability that the vehicle adheres to the lane change message relayed from the controller.

The logic of action 2 is to provide gaps on lane 1 by incorporating lane 2 in the merge process. Lane 2 has the potential to accept vehicles onto the platoons and thus it can reduce congestion in the 
merge area. The platoon lanes cannot accept all vehicles from lane 1 as it is assumed that vehicles in lane 1 did not intend to travel in platoons. Therefore, the lane change from lane 1 to lane 2 , is executed for every individual merging vehicle separately. The required lane changes provide the gap for every merging car.

The lane changes from lane 1 to lane 2, can provide the safe gap required for merge and prevent the prospective deceleration through the course of action 1. In this research it is assumed that the compliant vehicles on lane 1 can join the front or end of a platoon considering the certain prerequisites. In the next section the requirements for joining a platoon are presented. Figure 17 displays the specifications of the merge area parameters.

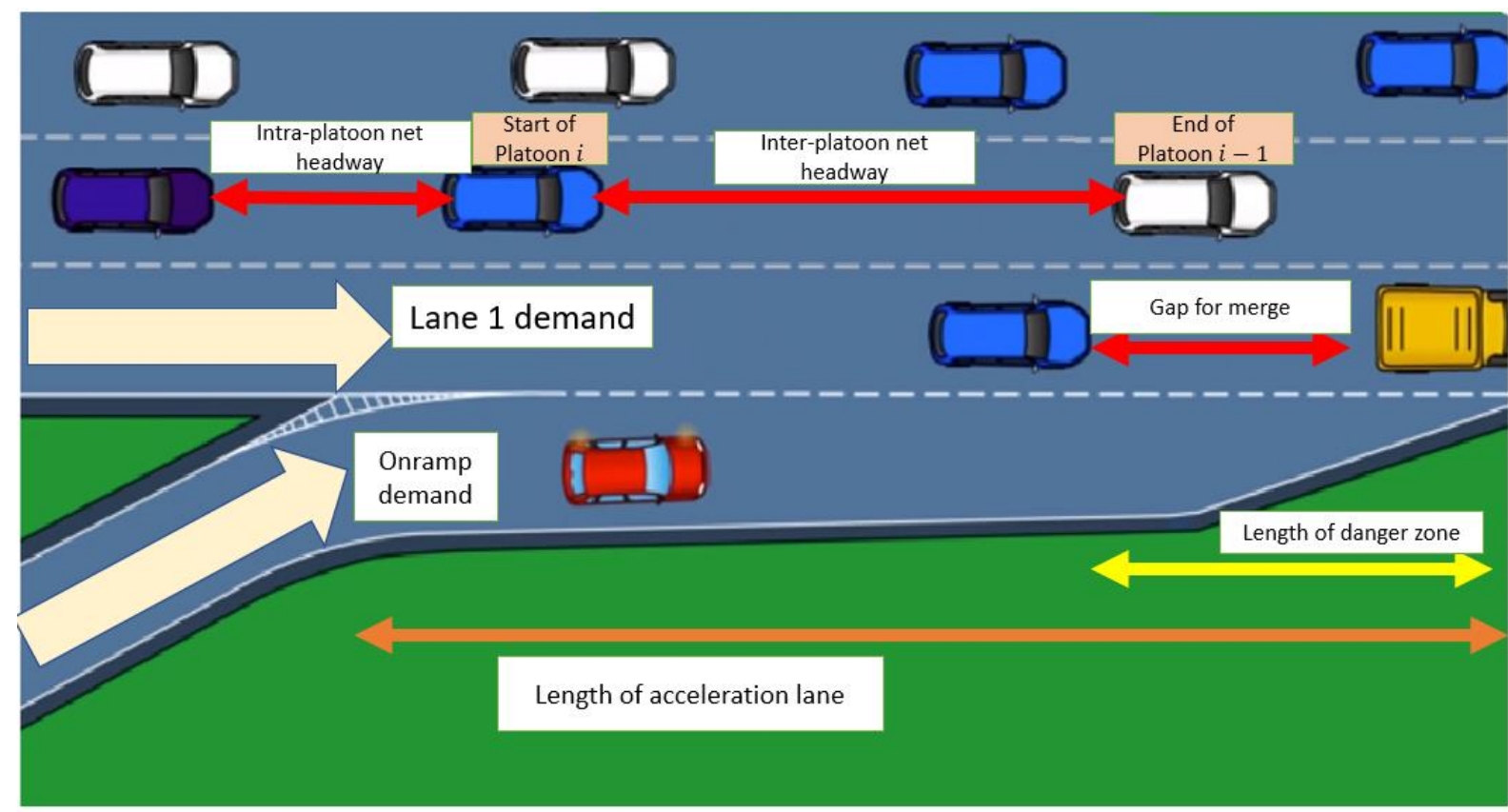

Figure 17 Components of the merge area

\section{Assumptions}

With increasing demand on lane 1, the average length of gaps in lane 1 will gradually decline. This reduction of average headways on lane 1 alongside a high demand on the onramp leads to the necessity of action 1 for gap creation on lane 1. Increasing numbers of action 1 negatively effects the traffic performance on this lane. Action 2 is triggered when the demand for lane 1 is higher than its capacity. Therefore, the demand measurement of lane 1 and onramp determines when action 2 is required. The aim of action 2 is to reduce the number of required actions 1 on lane 1 via enabling lane 
change from lane 1 to lane 2 . The operation of action 2 can reduce the traffic disturbances by action 1 on lane 1.

\section{Expected Improvements from Action 2}

With the activation of action 2 and vehicles on lane 1 moving to lane 2-for gap creation on lane 1less frequency of action 1 is expected. Fewer lane 1 actions yield less perturbations created by deceleration on lane 1 and can increase the onramp merging capacity and the onramp area throughput.

Conditions for the start of action 2 operation is the sum of the onramp and lane 1 demand being bigger than lane 1 capacity obtained in previous section as can be seen in Equation 12.

$$
q_{\text {merge }}+q_{\text {lane } 1}>Q_{\max (\text { lane } 1)} \quad \text { Equation } 12
$$

\section{Action 2 features}

The main features of action 2 are listed below:

- Action 2 targets at using lane 2 as means for throughput increase for the merging cars

- Action 2 serves alongside action 1 and occurs if lane change is feasible.

- Feasible lane change is joining the front or back of a platoon.

Action 2 is triggered based on the onramp demand measured by a sensor. This sensor is placed at a $100 \mathrm{~m}$ distance from the start of the acceleration lane.

If the lane change was not possible, the merging vehicle joins a merging gap which is available or created by action 1 .

\section{Detection Point}

The detection point of onramp vehicles in action 2 and 3 in this study is considered to be $100 \mathrm{~m}$ before the acceleration lane. This length requires the onramp to have at least 100 meters upstream of the acceleration lane. This length was selected to provide the time required for the processing of the traffic by the controller, target vehicle on lane 1 to be selected and the following potential action to take place. Figure 18 displays a schematic of the approximate position that the onramp vehicle will be identified on a typical geometry. 


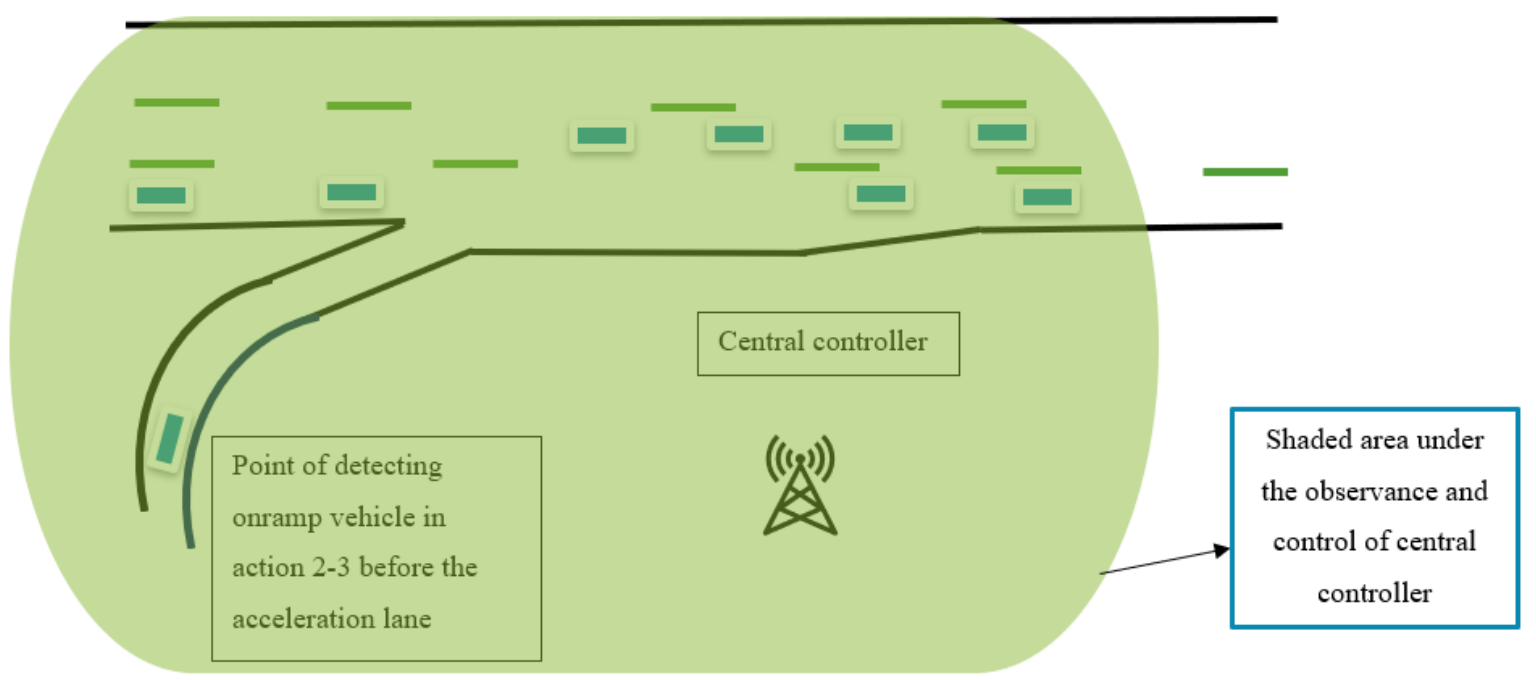

Figure 18 Observance and processing area for the controller

\section{Action 2 Process}

For every merging vehicle passing the sensor on the onramp, the potential gaps on lane 1 are detected by the central controller. If any of the moving gaps on lane 1 which are within range according to the movement of the merging vehicle, are not long enough for merge, a target vehicle on lane 1 is selected within the specific range to move to lane 2 . In this moment the action 2 process is triggered. The target vehicle identifies the platoons and if they are in the range of the table above, the lane change is possible. The target vehicle has a compliance rate which determines it adherence probability to join lane 2 . This compliance rate is an input in the model and set at 0.8 . If the target vehicle is compliant, the lane change occurs, and the required gap is created for the merging vehicle. Figure 19 display the merge process with the operation of actions 1-2. 


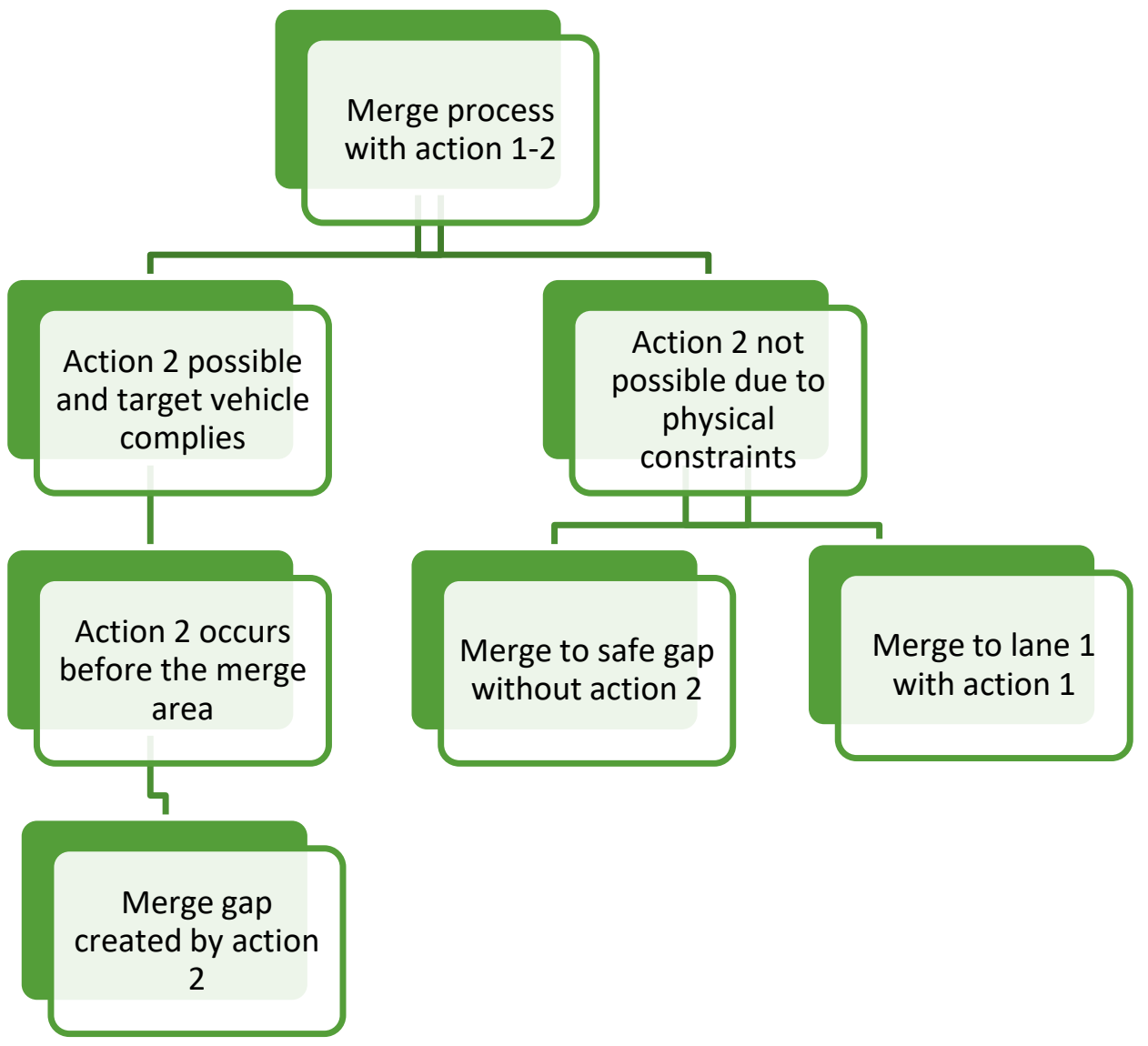

Figure 19 The merge process with the operation of action $1 \& 2$

\section{Joining a platoon}

A vehicle can join the front or back of a platoon, therefore no vehicle breaks into the middle of a platoon. Action 2 requires a vehicle from lane 1 to join the front or end of a platoon in lane 2 . The conditions below are required for the completion of this action:

- Feasibility of joining a platoon

The vehicle in lane 1 must be within the suitable distance for joining a platoon

- Target vehicle compliance to joining a platoon

The vehicle on lane 1 must be compliant to the recommendation of action 
Assigning a target vehicle to change lane for gap creation does not utilize maximum capacity of lane 2 but replaces potential action 1 with action 2. In the operation of action 2, the target vehicle on lane 1 has the longitudinal position of $\boldsymbol{X}_{\boldsymbol{m}}$ when the action is initialized. The platoons on lane 2 are observed by the central controller. The front of a platoon is displayed with $\boldsymbol{X}_{\text {plt }}$ front while the end of a platoon is displayed with $\boldsymbol{X}_{\boldsymbol{p l t}} \boldsymbol{t}_{\text {end }}$. If the position of the target vehicle is within one of the ranges displayed in the table below, the target vehicle can join the respective platoon. This range was selected for this study as it is assumed to be the minimum range for the lane change possibility. The compliance rate considered in this study was 85 percent.

Table 4 displays the conditions for a vehicle joining a platoon. It is created with the assumption that a vehicle will join the front or end of a platoon, disabling the possibility of joining the middle of a platoon. The basis for the selection above was to make joining platoons feasible in terms of time and physical conditions.

\section{Table 4 The requirement for each type of action 2}

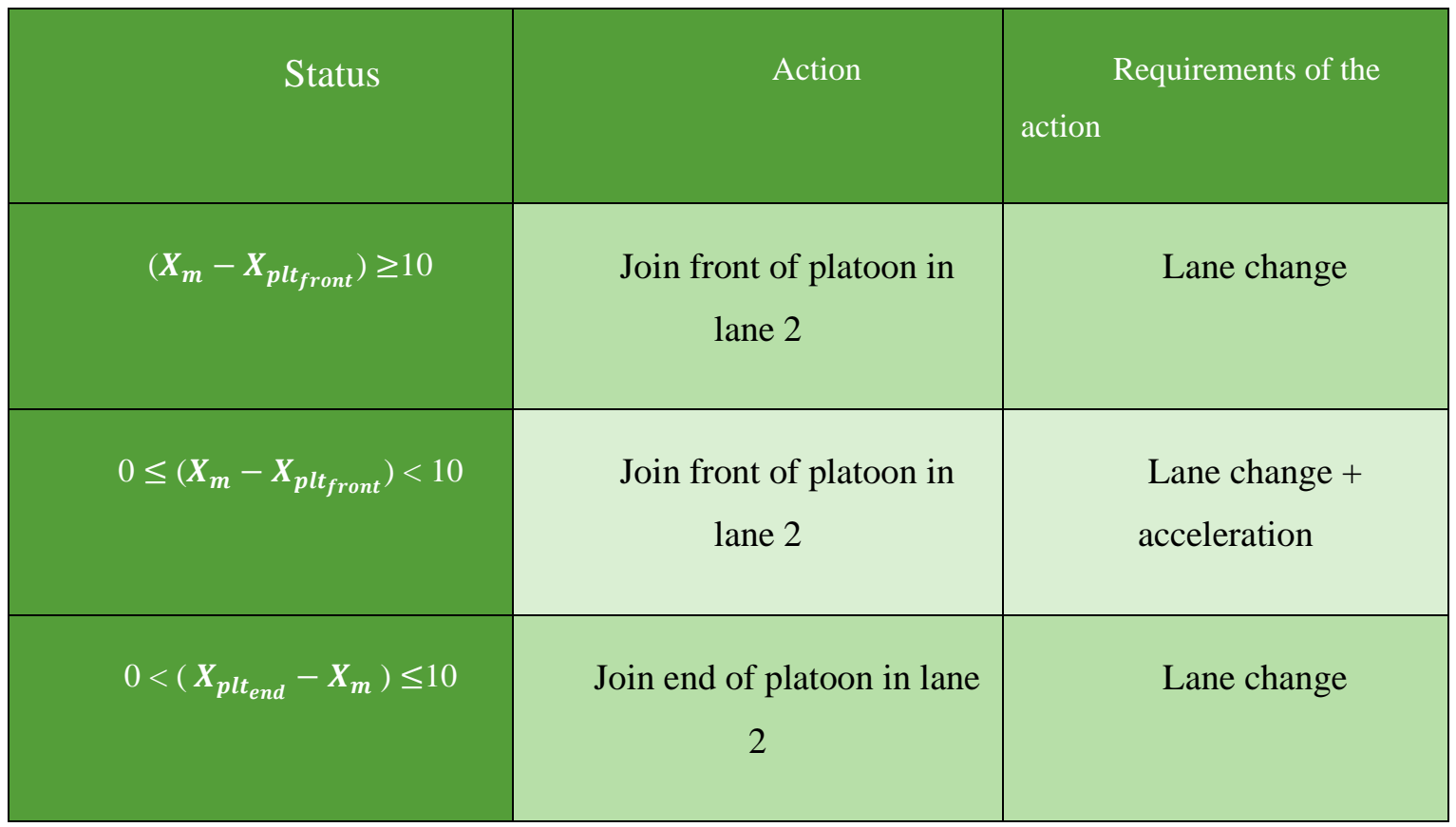

The pseudocode of the steps for this merge, applied in the simulation is;

FOR follower vehicle $i$ on the non-platoon lane 
Identify the platoon $i$ on lane 2 which has the least distance to the vehicle Min |PltPos -VehPos| IF ( VehPos - PltPos $>0)$

CAV merges to platoon and becomes leader of Plt

ELSE IF (PltEnd - VehPos > 10m)

$C A V$ merges to platoon and joins end of Plt

END IF

END FOR

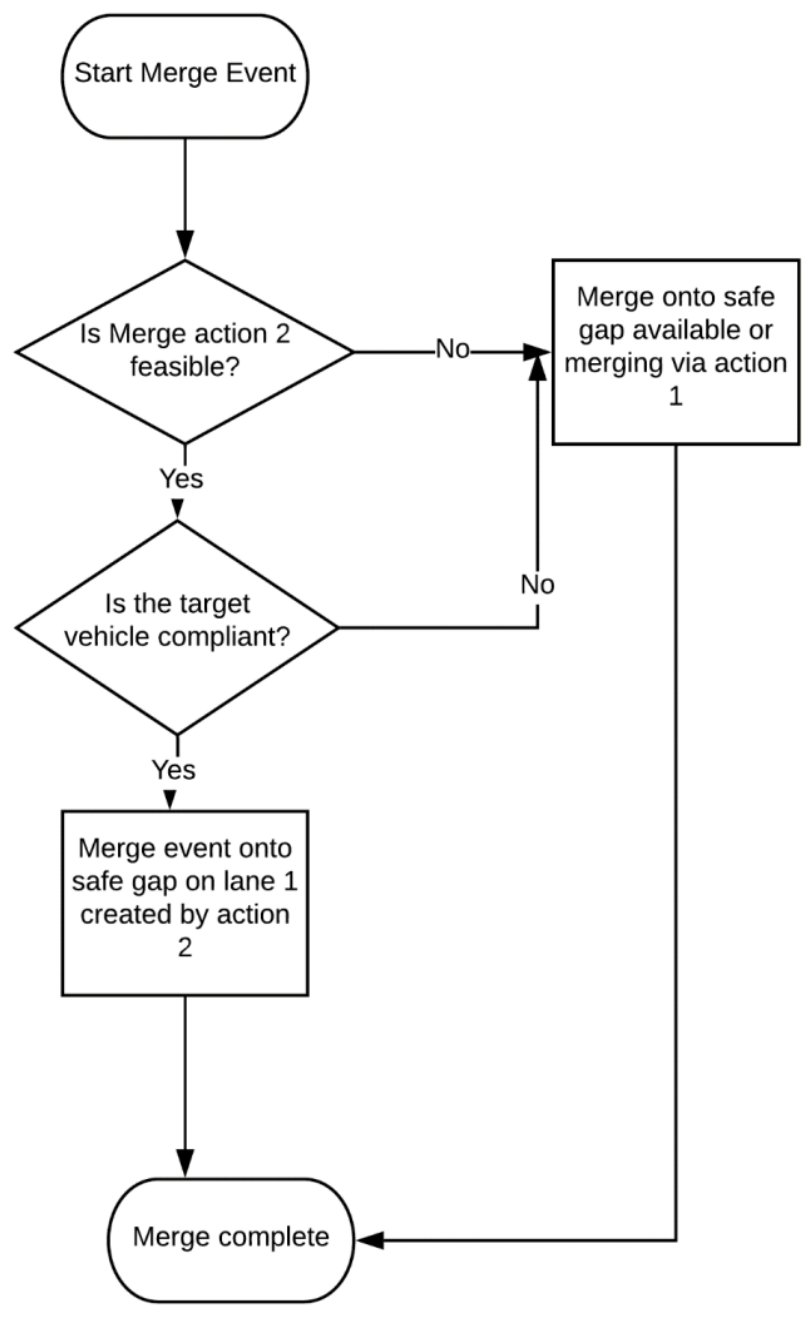

Figure 20 Steps for the completion of action 2 


\subsubsection{Merging with Action 3}

In action 3, similar to action 2, two lane change movements will be utilized to assist the merge area throughput. Selected target vehicle on lane 1 will join a platoon in lane 2, while a vehicle on lane 2 will join a platoon on lane 3 . The merging vehicle is detected before entering the acceleration lane. The onramp vehicle is detected by roadside sensor $100 \mathrm{~m}$ prior to the acceleration lane and the vehicles information is relayed to the central controller. The central controller detects the gaps feasible for merging on lane 1. If a suitable gap for merge is not available, a specific target vehicle is selected to move lane from lane 1 to lane 2. Vehicles respond to the relayed message based on a defined compliance rate. If the compliant vehicle cannot join lane 2 because of their relative distances, a vehicle on the selected platoon on lane 2 moves to a platoon in lane 3 . The same perquisites for lane change from lane 1 to 2 are available for the lane change from lane 2 to lane 3.

The logic of action 3 is to provide gaps on lane 1 by incorporating lane 3 beside lane 2 in the merge process. Lane 3 has the potential to accept vehicles onto the platoons and thus it can reduce congestion in the merge area.

The lane changes from lane 2 to lane 3, can provide the safe gap required for merge and prevent the prospective deceleration through the course of action 1. In this action scenario, the two platoons which will be exchanging vehicles must be within the length feasible for the lane change. Table 5 displays the proximity requirements of the two platoon positions for the occurrence of the lane change. The $10 \mathrm{~m}$ distance displayed in table below is the minimum required distance considered in this model for enabling the lane change from one platoon to another.

Table 5 displays the steps for the completion of merge with the operation of actions 1-3.

Table 5 The conditions for a vehicle to join from a platoon in lane 3 from lane 2

\begin{tabular}{|c|c|c|}
\hline Status & $X_{p l t_{\text {Lane2 (end) }}}-X_{\text {plt }}$ tane3(front) $>10$ & 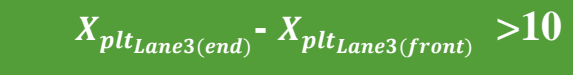 \\
\hline Action & $\begin{array}{l}\text { Vehicle from platoon in lane } \\
2 \text { joins front of platoon in lane } 3\end{array}$ & $\begin{array}{l}\text { Vehicle from platoon in lane } \\
2 \text { joins end of platoon in lane } 3\end{array}$ \\
\hline
\end{tabular}




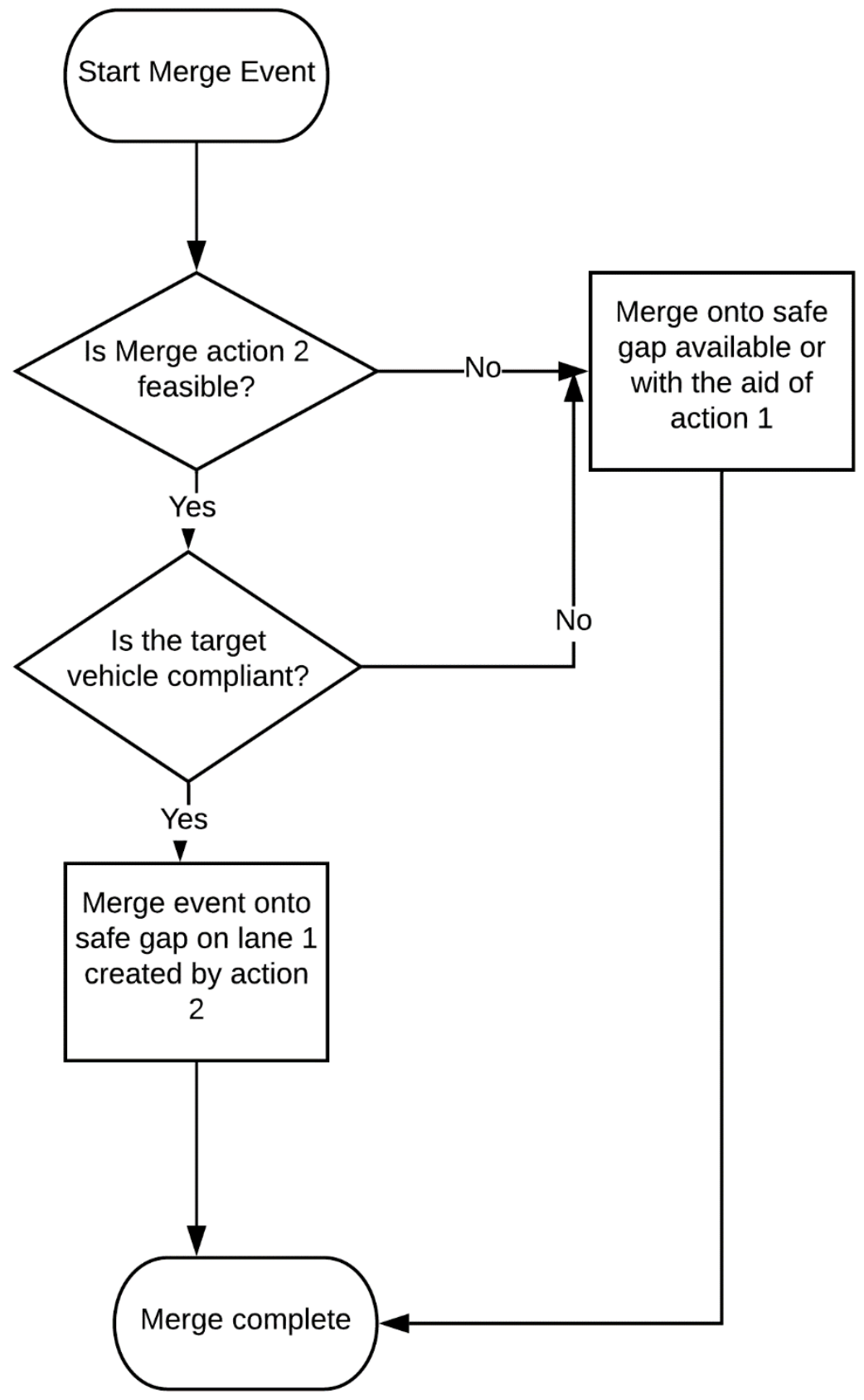

Figure 21 Steps for the operation of action 3 


\section{Table of notations}

In Table 7-9 the parameters of the car following model, the merge model, and the traffic performance are displayed. The notations are listed according to the class of the parameter.

\section{Parameters of the Car Following Models}

\section{Table 6 The notations of the parameters in the car following model}

\begin{tabular}{|c|c|}
\hline Parameter notation & Definition \\
\hline $\mathrm{H}$ & Headway (time) \\
\hline $\mathrm{i}$ & Intra-platoon headway, i \\
\hline $\mathrm{h}$ & Inter-platoon headway, h \\
\hline$q_{\text {ramp }}$ & Demand Flow Onramp (veh/hr) \\
\hline$q_{\text {lane } 1}$ & Demand Flow Lane 1 (veh/hr) \\
\hline $\boldsymbol{q}_{(p l t)}$ & Flow rate for the platoon lane $(\mathrm{veh} / \mathrm{hr})$ \\
\hline$q_{\text {merge }}$ & Onramp Merge (veh/hr) \\
\hline$q_{(1)}$ & Throughput of lane 1 from lane 1 demand \\
\hline$q_{\text {total }}$ & Total demand of lane 1 and onramp \\
\hline $\mathrm{n}$ & Number of Vehicles in a Platoon (\#) \\
\hline
\end{tabular}


Table of Notations for the Rule Based Model Parameters

Table 7 The notation of parameters in the model

\begin{tabular}{|c|c|}
\hline Parameter notation & Definition \\
\hline$S_{\text {merge }}$ & Minimum Safe Gap for Merge (m) \\
\hline$S_{t}$ & Gap Available (m) at time $t$ \\
\hline$L_{d z}$ & Length of Danger Zone \\
\hline$S_{\max (t)}$ & Max Gap for Merge \\
\hline$S_{\max (t+1)}$ & Next Max Gap \\
\hline$S_{\text {safe }_{(\text {мовIL) }}}$ & Safe Gap for Merge According to MOBIL model \\
\hline$T_{\text {action }}$ & Time Required for Gap Creation \\
\hline
\end{tabular}

Table of Notations for the Traffic Performance Measure Parameters

Table 8 The notation of traffic performance measures in this study

\begin{tabular}{|c|l|}
\hline Parameter notation & Definition \\
\hline $\boldsymbol{Q}_{\max (\text { lane 1) }}$ & Maximum Throughput Lane 1 (veh/hr) \\
\hline $\boldsymbol{Q}_{\max (\boldsymbol{M} \boldsymbol{A})}$ & Maximum Merge Area Throughput (veh/hr) \\
\hline
\end{tabular}


Table of Notations for the Geometric Parameters

Table 9 The notation of the length of acceleration lane

\begin{tabular}{|c|l|}
\hline Parameter notation & Definition \\
\hline $\boldsymbol{L}_{\text {accl }}$ & Length of Acceleration Lane \\
\hline
\end{tabular}




\subsection{Merging Event}

A merge event is defined in this research as the start of a vehicle on the acceleration lane until the merging of an onramp vehicle onto the simulated highway.

Every onramp vehicle on the acceleration lane detects the largest gap, $S_{\max (t)}$, which is feasible to join on lane 1 . If at any timestep the $S_{\max _{(t)}}$ is larger than the minimum safe gap for merge, $S_{\text {merge }}$, (which is obtained from the MOBIL lane change model), the merging vehicle merges onto the gap. If the merging vehicle cannot merge within a specific distance from the end of the acceleration lane, an action must occur on lane 1 to assist the merge.

Through V2I communication, the target vehicle for the action in the mainlines is determined. Overall, the upstream vehicles can take three types of actions to assist the merge process:

(i) Action 1: A vehicle on Lane 1 may decelerate to create a gap feasible for the merge

(ii) Action 2: A vehicle on Lane 1 may switch over to the left adjacent platoon lane (Lane 2) to create more space for vehicles in Lane 1 and onramp vehicles; and

(iii) Action 3: Action 2 plus a vehicle on Lane 2 may switch over to Lane 3.

The actions are triggered if an onramp vehicle is unable to safely merge (and is projected to come to a stop towards the end of the acceleration lane). Action 1 is triggered when an onramp vehicle is on the acceleration lane. Action 2 and 3 take place within a segment upstream action 1 and are triggered based on the onramp demand measured by a sensor before the acceleration lane. Action 3 provides lane 3 for the lane change of mainline vehicles to further assist the lane change process.

It must be noted that when action 2 and 3 are operating, ideally the model seeks to provide gaps for merging vehicles through action 2 and 3 . If the actions 2 and 3 are not able to provide the necessary gap, then action 1 is required. Therefore, action 2 and 3 have priority over action 1, as they do not cause disturbances to the mainline traffic flow. 
In every merge event, the model outputs displayed below are recorded, presenting the record of events in that simulation run. The first column shows the type of action assisting the merge, which can be 1,2 , or 3 . The second column displays whether a lane change occurred in the merge event. If the action was 2 or 3 , the amount recorded in the lane change column will be 1 , otherwise it will be 0 . The last column displays the deceleration rate require for action 1.

\begin{tabular}{|l|c|c|}
\hline Merge type & Lane change & Deceleration by action 1 \\
\hline $\mathbf{X}$ & 1 or 0 & B \\
\hline
\end{tabular}

\subsubsection{State- Action for an event}

The system state represents the traffic flow conditions upstream the merge area when the merge event starts. The system state is utilized by the controller, processed, and then informs the type of action to the relevant action vehicles. Additionally, the system state determines which type of actions should be active in every time period.

The system real-time state is important as it has an overview of the traffic conditions upstream the merge area and therefore that observation can be exploited to make the type of action decision based on the system optimum. The real time information is collected through sensors which are capable of detecting speeds, density and headways of the traffic. The real-time information received by the controller is processed and returns the suitable action for gap creation on lane 1.

The system state can be defined by density, average headways, average speeds, average platoon lengths, etc. The type of merge action is selected such that the least disturbance occurs on the mainlines or in other words the estimate for the system state performance is maximized.

In this research the selection of action (deceleration on lane 1 or joining a platoon in adjacent lane) is based on the density on lane 1 (lane 1 demand), onramp demand, as displayed in figure below.

The flowchart in Figure 22 displays the steps for a merge action completion. 


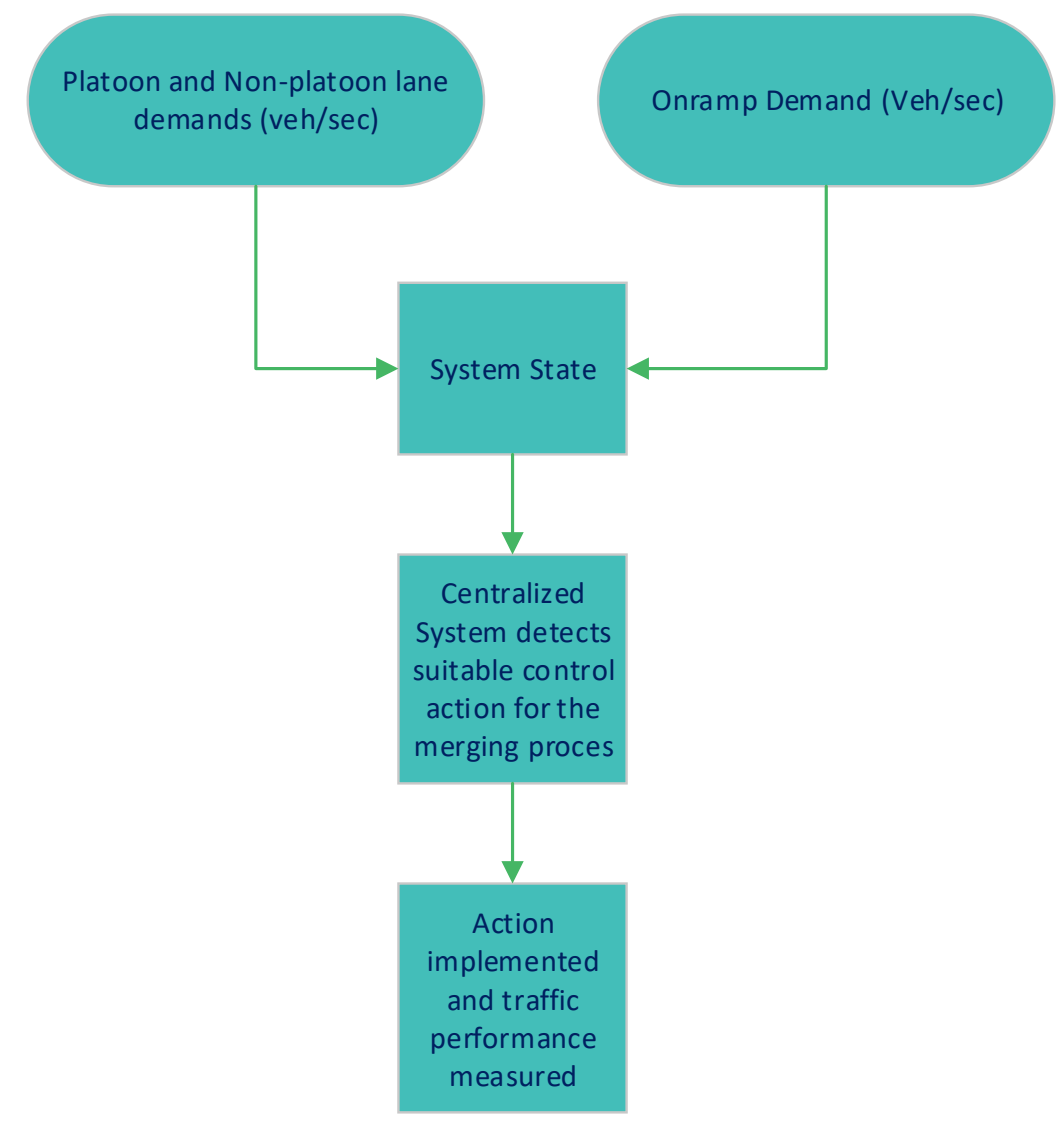

Figure 22 The process for a merge to finish

\subsubsection{Relation between the merge and traffic factors}

In every merge event in this model, the major actors which determine the outcome of the event are:

1. Demand on lane 1 (average headway on lane 1)

2. Onramp vehicle demand (arrival rate onramp)

3. Minimum gap required for the merge (according to MOBIL for IDM)

4. Maximum available gap for the merging (for every onramp vehicle)

5. Percentage of gaps requiring no further projection or action (suitable for merge)

According to traffic fundamentals, the range of headways in lane 1 is within a range which has a direct relation with the lane 1 demand. The average headways in lane 1 can be defined as a function of 
the lane 1 demand as can be seen in Equation13-14. The same applies for the average headways of onramp vehicles.

$$
\begin{array}{ll}
\bar{H}_{m} \propto F\left(q_{\text {lane } 1}\right) & \text { Equation } 13 \\
H_{1} \leq \bar{H}_{m} \leq H_{2} & \text { Equation } 14
\end{array}
$$

Where $\bar{H}_{m}$ is the median headway, $q_{\text {lane } 1}$ is the demand volume and $\left[H_{1}, H_{2}\right]$ are the lower and upper bounds of the headway range.

The minimum gap required for merging in the model, $S_{\text {merge }}$ is according to MOBIL lane change model based on IDM car following.

$$
S_{\text {merge }} \geq S_{\text {safe }_{(\text {мовІL })} \quad \text { Equation } 15}
$$

Where $S_{\text {merge }}$ is the minimum available gap for merge and $S_{\text {safe }} e_{(\text {MOBIL })}$ is the minimum required gap according to the MOBIL model. The available gaps displayed as $S_{\max (t)}$ in this research, are dependent on the traffic demand and speed range of the traffic (mainline). While the $S_{\text {Safe }} e_{(\text {Mов IL })}$ is dependent on the average traffic speed and the car following model.

In every timestep, the maximum available gap for merging, $S_{\max (t)}$, for an onramp vehicle is the maximum gap within the feasible range for the onramp vehicle to join according to Equation 16.

If the equation below was valid in that timestep for the merging vehicle,

$$
S_{\max (t)} \geq S_{\text {Safe }_{(\text {мовIL })}}=\min \left(S_{\text {merge }}\right) \quad \text { Equation } 16
$$

Then the merging would occur with no required action on lane 1.

\section{Demand and Merging Relations}

The percentage of gaps requiring no further projection or action (suitable for merge) depend on the headways in lane 1 as can be seen in equation below:

$$
S_{\text {Safe }_{(\text {мовІL })}} \propto\left\{\bar{H}_{m}\right\} \quad \text { Equation } 17
$$


In which $\bar{H}_{m}$ is the average headway between vehicles on lane 1 .

And since $\bar{H}_{m}$ is a function of the demand (Equation 18), therefore:

$$
S_{\text {Safe }_{(\text {мовІL })}} \propto F\left(q_{\text {demand }}\right) \quad \text { Equation } 18
$$

The more demand on lane 1, the shorter the average gaps for merging available and thus less possibility for a gap to be sufficiently large (according to the merge model specification) for merge. (Equations 19-20)

$$
\begin{aligned}
& \uparrow q_{\text {demand }} \Rightarrow \downarrow P\left(S_{\max (t)} \geq S_{\text {safe }_{(\text {MовIL })}}\right) \quad \text { Equation } 19 \\
& \uparrow q_{\text {demand }} \Rightarrow \downarrow \bar{H}_{m} \Rightarrow \downarrow P\left(S_{\max (t)} \geq S_{\text {safe }_{(\text {MOBIL })}}\right) \quad \text { Equation } 20
\end{aligned}
$$

Additionally, lower headways on lane 1, lead to higher mainline traffic which would enforce actions-affecting the mainline throughput-for creating suitable gaps on the mainlines. The merge area capacity can be displayed as a function of the demands, traffic flow behavior, and actions for merge (Equation 21).

$$
Q_{\max (\text { lane } 1)} \propto F\left(V_{\text {demand }}, \bar{S}_{m}, S_{\text {safe } \left._{(\text {мов } I L)}\right)}\right) \quad \text { Equation } 21
$$

As the parameters of each section have a specific influence on the merging actions, the models merge performance can be summarized as a function of the control system, the car following model and the highway geometry:

Merge area performance $=f($ Control System Strategy, Car Following, Highway

\section{Geometry)}

Since higher demands on the mainline provide less suitable gaps for merging vehicles, it is important to consider the tradeoff between headways and availability of gaps on lane 1. A shortage of gaps on the mainline, in the merge area proposed in this research, triggers actions which potentially affects the mainline traffic.

Higher onramp vehicle demand requires more frequent gaps on the mainlines. As discussed in this section, the frequency of merging gaps relates to the demand on the lane 1 of the traffic. The target of this merge model is to create a mechanism for maximizing the mainline throughput while maintaining the maximum merging possibility through merging (with and without required actions) on the mainlines. This mechanism implemented through the Rule Based merging model can be described as 
similar to the Fundamental Law of Gearing, where the mainline traffic and onramp merging vehicles are each a gear and the tooth are the traffic flowing.

\subsubsection{Differences between the merging types}

The differences in the merge types simulated in this study are the type of action on the mainlines creating the gap for merge. It is also important to note that this model has flexibility for implementation of different car followings and adjustments to lane types, which means actions can be set to different lane configurations. The only difference between action 2 and 3 are the availability of lane 3 for lane change in this action. Table 10 displays the differences between merge types.

Table 10 Differences between merge types

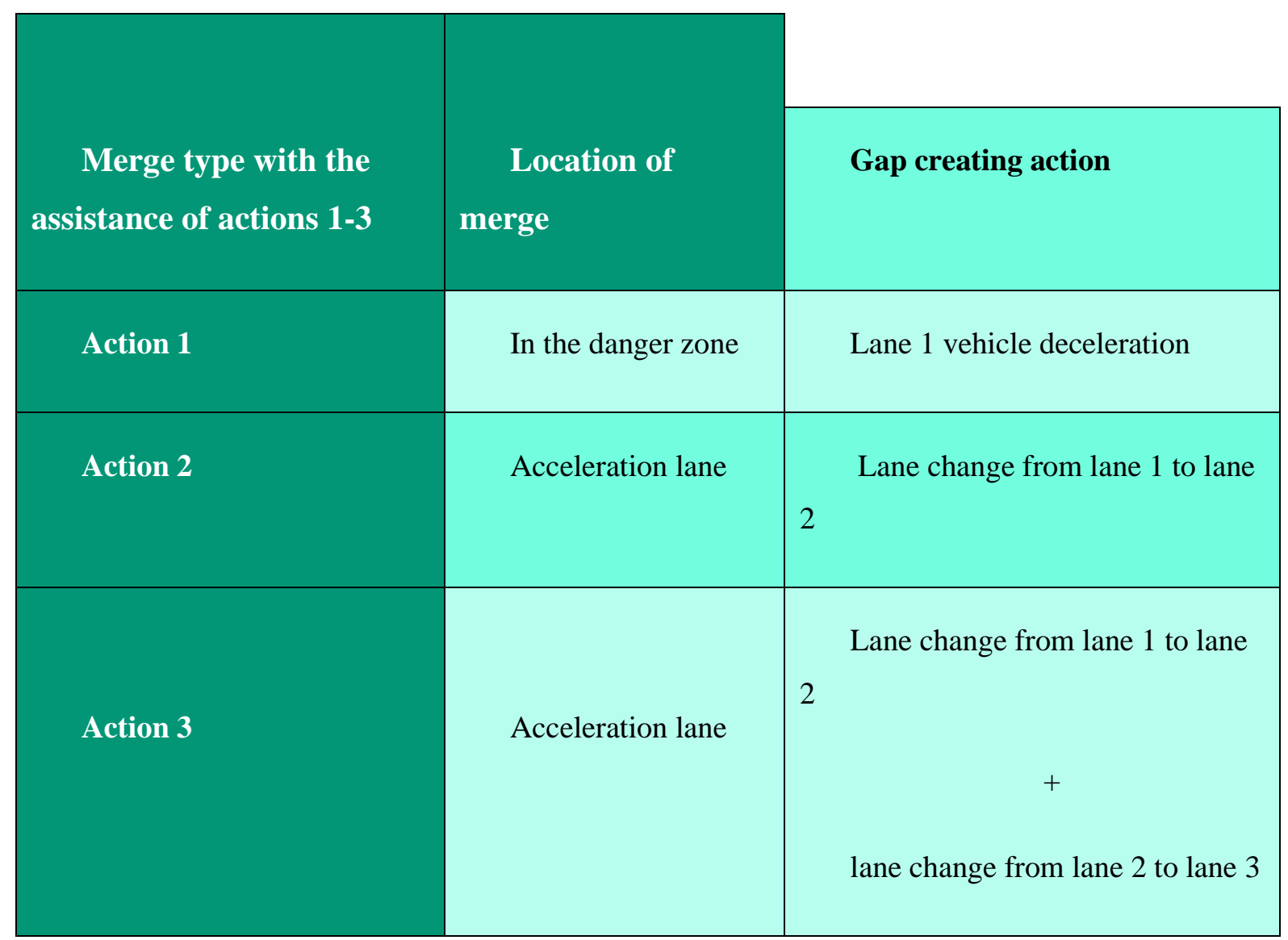

The differences in the proposed merge types are in, the time of merge (when does the merge event start), the vehicles controlled (onramp vehicle, mainline vehicles) in the actions and the 
thresholds for the controls within the action. The similarities between the merge types will be the IDM+ car following in non-platoon lanes, the platoons in merge types $1 \& 3$, ramp length, generated speed. The expected difference between the merge types are, Travel Times (system), Merge area capacity, Maximum vehicles merged.

The calibration for optimization of the results will include setting the relevant parameters to maximize the gain of the system. This includes the manipulation of, desired speeds, car following parameters, safety gaps, geometry features of the merge area such that the desired outputs of the objective function are maximized. The objectives include, average travel time, throughput, least frequency of actions 1 (least cost incurred from actions).

The model results obtained can be versatile with the control of relevant attributes which enable it to become modified for any specific location. One other advantage of this model is the versatility of the model in replacing the car followings of the lanes.

The information obtained from this model can be useful for controlling merge points and having more capacity of flow downstream the merge area.

\subsection{Simulation Experiments}

For obtaining valid results from the simulation model, it is essential to have the acceptable amount of the relevant factors. This objective can be achieved according to testing the factors below by methods such as exhaustive search to yield better performances:

- Control strategy parameters (thresholds)

- Car following parameters

- Road geometry parameters

Various attributes of the model play a role in the merging action and thus their sensitivity must be investigated. These attributes include, role of acceleration lane length on number of actions in a certain time period, system ability to pass maximum demand on mainline and ramp, impacts of higher onramp demand on system performance with different control actions. Other significant parameters in affecting the merge area performance are:

- Length of speeding lane; higher length increases the possibility of merge without action 
- Onramp demand and mainline demands

- Regular Lane characteristics (car following, stochastic entrance, deterministic entrance)

- Platoon Lane characteristics (car following, stochastic entrance, deterministic entrance)

- Average Speeds on mainline

The simulation model is created in $\mathrm{R}$ with producing the necessary parameters for the simulation of the merge area. The car following for non-platoon lanes is (IDM+) called at every timestep to process the movement of the vehicles. The platoon lanes have a separate car following function to update their positions, speeds, and accelerations. The focus of the simulation is the merge area throughputs and travel times and thus the specific vehicle dynamics and car followings are not under investigation The traffic performance measures which can be measured from the simulations are, Merge Throughput, Merge Area Capacity, Travel Times, Average Speeds, Average Densities, and Average Headways.

The simulation tests are intended to measure the traffic performance differences between the proposed merge types and geometric conditions alongside analyzing the pattern of behavior with different car following parameters.

\subsubsection{Traffic Demand}

The traffic demand is a key parameter in the simulations as it would determine the frequency of actions, the maximum throughputs, the merge capacity etc. The demand of the onramp and lane 1 traffic were inserted in the model as ranges for the headway of the vehicles. The demand for the platoon lanes were inserted as platoon lengths within the model inputs. The generation of every vehicle on lane 1 and onramp would be $t$ seconds after the previous vehicle. The $t$ would be randomly selected within a normal distribution range with lower and upper bounds of $\left[t_{1}, t_{2}\right]$. This $t$ will be the headways of the vehicles at generation, but the headways of vehicles at the merge area would not necessarily be in the same range, as vehicles have different desired speeds. The desired free-flow speeds of vehicles were also inserted in the model as a range, $\left[v_{1}, v_{2}\right]$. The free-flow speed of every vehicle would be a number normal random selected in this range. 


\subsubsection{Constraints of the model}

The model is subject to certain constraints. The main constraints in the development and operation of the model are listed below:

- The modified IDM+ minimum operational headway (average 1.1 seconds)

- The control strategy designed to assist the merge separately for every vehicle

- Three lanes in the merge area segment

\section{System inputs to prevent breakdown}

To prevent the breakdown and ensure the functionality and robustness of the model in the simulation tests, there are certain prerequisites for the model inputs. The prerequisites are defined based on the model design and operation. The conditions of the model to prevent breakdown on lane 1 are listed below:

1- The minimum safe gap for merge must be longer than the minimum headway between vehicles in lane 1

Minimum Safe Gap for Merge $=S_{\text {merge }}$,

Minimum headway to enable onramp merge $=H_{\text {SafeGap }}$

$X_{\text {SafeGap }} \propto H_{\text {SafeGap }}$, safe gap for merging is relevant to a safe headway between following vehicles

$$
H_{\text {Aveonramp }}>H_{\text {SafeGap }} \quad \text { Equation } 22
$$

The condition above must be implemented to prevent uncontrollable congestion on lane 1 which leads to breakdown. If the condition above is not met, the excess merging vehicles cannot merge and will cause breakdown on the onramp.

2- The minimum headway between onramp vehicles must be larger than the minimum headway between vehicles on lane 1
A) $\operatorname{Max}\left(H_{\text {Avelane } 1}\right)<\operatorname{Min}\left(H_{\text {Aveonramp }}\right)$
Equation 23
B) $\operatorname{Max}\left(H_{\text {Avelane } 1}\right)>\left(H_{\text {SafeGap }}\right)$
Equation 24 
Condition (A) above must be implemented to prevent 2 onramp vehicles approaching before 2 mainline vehicles. This is due to the gap assignment of 1 vehicle per event. Additionally, this condition prevents onramp flow exceeding lane 1 flow. The conditions above are set for all the simulations tests in this research. For condition (A), the base setting for the headway range on lane 1 was [1.4-1.9] seconds while the headway range for onramp vehicles was [2.5-3] seconds. For condition B, the $H_{\text {SafeGap }}$ is 1.4 seconds and the maximum headway between lane 1 vehicles is 1.9 seconds.

Condition (B) enables gaps larger than the safe gap to be created and thus prevents all merging cars to require an action on lane 1.

\subsubsection{Simulation set up}

In order to obtain the influence of parameters on the merge model performance, the tests on the varying following parameters were implemented:

- Mainline and onramp demand

- Variation of onramp demand ranging from 900 to $1400 \mathrm{vph}$ and mainline volumes at base $2180 \mathrm{vph}$

- Platoon length variation from 2 to 10 vehicles

$\circ$ Interplatoon headway range from 2 to 3 seconds

- Model and geometric parameters

- Analysis of the safe gap for merge starting at $35 \mathrm{~m}$,

- Analysis of the acceleration lane length starting at $150 \mathrm{~m}$

The addition of each action provides the model with more utility of the mainlines for assisting the merge. In order to test the performance of the merging model, the approach was to utilize the efficiency of each action separately. Thus, the solution approach was to add the potential benefits of each action in a step by step approach. The Solution approach (methodology) for the model is:

- Slowly increase mainline and onramp demand while

O Only action \#1 is allowed 
$\circ \quad$ Only action \#1 and 2

- All actions are possible

This approach helped in obtaining the thresholds for each action to be activated and the level that each action can assist the merge area. For each simulation, the collected performance measures include the number of lane changes, delays/travel times, throughput, and travel time for onramp vehicles.

Each of the inputs affect the output dependently or independently. In the geometric class of parameters, the length of the acceleration lane is considered for analysis. For the control model parameters, the minimum safe gap for merge and the platoon lane characteristics were selected. Other control parameters such as location of sensing, were not tested as they would not affect the output of the action.

Table 11 displays the parameters which are analyzed in the next section and their influence on other parameters and the merge area. The frequency of actions in the simulation results are important as they are a display of how the model is operating. With the sole operation of action 1 , the model is performing better with lower frequency of action 1 to be required for merge. When action 2 and 3 are also active, the model's performance is better when higher percentage of merging vehicles merge with the assistance of action 2 or 3 . The basis of this rationale is reducing disturbances to the flow on lane 1. 
Table 11 Classification of the parameters of the model

\begin{tabular}{|c|c|c|}
\hline \multicolumn{2}{|c|}{ Class of parameter } & \multirow{2}{*}{$\begin{array}{l}\text { Effects on Control Actions and } \\
\text { Throughput } \\
\text { Frequency of action } 1\end{array}$} \\
\hline Geometric & $\begin{array}{l}\text { Length of acceleration } \\
\text { lane }\end{array}$ & \\
\hline \multirow{2}{*}{ Control Model } & $\begin{array}{l}\text { Minimum safe gap for } \\
\text { merge }\end{array}$ & Frequency of actions 1,2 and 3 \\
\hline & $\begin{array}{c}\text { Platoon } \\
\text { lengths/headways }\end{array}$ & Frequency of action 2 and 3 \\
\hline \multirow{3}{*}{$\begin{array}{l}\text { Car Following } \\
\text { Model }\end{array}$} & Onramp demand & Merge area throughput \\
\hline & Demand on Lane 1 & $\begin{array}{l}\text { Frequency of actions } 1,2 \text { and } 3 \\
\text { Throughput of lane } 1\end{array}$ \\
\hline & Detection Zone on lane 1 & Frequency of actions 1,2 and 3 \\
\hline
\end{tabular}

\subsubsection{Model Verification}

Model verification was achieved by running simulations of the platoon and non-platoon lanes with the designated car following features and obtaining the results within the acceptable range considering the random parameters. The verifications are completed in order to verify the flow on lane 1 and the platoon lanes.

\section{Lane 1 (non-platoon) Flow Specifications}

The characteristics of the traffic flow on lane 1 are listed below: 
- Car following Model in lane 1: (IDM+)

- Headway range (sec): [1.4-1.9] second in order to replicate current day traffic

- Desired speeds (m/s): [36-38] equal to approximately $80 \mathrm{mph}$ which is the maximum design speed of current day infrastructure

To verify the flow on lane 1, the throughput of traffic on lane 1 before the merge area is compared to the expected throughput of that lane. Table 12 displays the comparison of throughput for the verification of traffic flow on lane 1.

\section{Table 12 Verification of flow on lane 1}

\begin{tabular}{|c|c|c|c|c|}
\hline \multirow{3}{*}{$\begin{array}{l}\text { Comparison of } \\
\text { model and calculated } \\
\text { throughputs }\end{array}$} & \multicolumn{2}{|c|}{$\begin{array}{l}\text { Expected Model } \\
\text { Throughput }\end{array}$} & \multicolumn{2}{|c|}{$\begin{array}{l}\text { Model output of throughput in } \\
\text { lane } 1\end{array}$} \\
\hline & $\begin{array}{l}\text { Average } \\
\text { headway } \\
(\mathrm{sec})\end{array}$ & $\begin{array}{l}\text { Expected } \\
\text { throughput } \\
\left(\frac{v e h}{h r}\right)\end{array}$ & $\begin{array}{l}\quad \text { Lane } 1 \\
\text { headway input } \\
\text { range (sec) }\end{array}$ & $\begin{array}{l}\text { Throughput of } \\
\text { model }\left(\frac{v e h}{h r}\right)\end{array}$ \\
\hline & 1.65 & 2182 & 1.9] & 2128 \\
\hline
\end{tabular}

\section{Verification of the platoon lane}

The characteristics of the traffic flow on platoon lanes are listed below:

- Platoon length range [2-10]

- Inter-platoon headway range (sec): [2-2.2]

- Intra-platoon headway (25m): $0.7 \mathrm{~s}$

- Desired speeds (m/s): [36-38] equal to approximately 80mph which is the maximum design speed of current day infrastructure

To verify the flow on the platoon lane, the throughput of traffic on lane 1 before the merge area is compared to the expected throughput of that lane. Table 13 displays the comparison of throughput for the verification of traffic flow on the platoon lane. 
Table 13 Verification of the platoon lane

\begin{tabular}{|c|c|c|}
\hline Comparison & $\begin{array}{l}\text { Expected Model } \\
\text { Throughput }\end{array}$ & $\begin{array}{l}\text { Model output of throughput on platoon } \\
\text { lane }\end{array}$ \\
\hline $\begin{array}{l}\text { of model and } \\
\text { calculated } \\
\text { throughputs }\end{array}$ & $\begin{array}{l}\text { Platoon specifications: } \\
\mathrm{N}=5, \mathrm{~h}=2.5 \mathrm{~s}, \mathrm{I}=30 \mathrm{~m}\end{array}$ & $\begin{array}{l}\text { Model input range for platoon lengths [3- } \\
\text { 7] Average throughput of model }\left(\frac{v e h}{h r}\right)\end{array}$ \\
\hline & 2800 & 2788 \\
\hline
\end{tabular}

\section{Base assumptions in the model:}

The Rule Based merge model presented in this study, requires assumptions due to the limitations of the study and the model. The assumptions below are considered for the model:

- Lane change incurs less cost than deceleration on lane 1, and thus the initial target is to obtain maximum merge area throughputs with the assistance of lane changes.

- The action to create gaps for merging vehicles will be deceleration on lane 1 until the demand of onramp and lane 1 vehicles exceeds the capacity. This will initially utilize lane 1 until it's maximum throughput and then utilize lanes 2 and 3 via actions 2-3.

- When onramp and lane 1 demand lead to the breakdown of flow on lane 1 , then actions 2-3 requiring planning on the mainlines are triggered.

- Unlike action 1, actions 2-3 occur upstream the merge area, when the merging vehicle is not on the acceleration lane.

- Actions 2-3 aim at providing merging gaps while preventing action 1 as the last resort.

- In action 2 and 3, all the targeted cars for the actions won't be complying to the controls of the central observer and thus action 1 may be required. 


\subsubsection{Expected Outcome of the Actions}

\section{Operation of action 1}

Action 1 creates gaps on lane 1 for the merging vehicles through the deceleration of lane 1 vehicles. The effect of deceleration on lane 1 results in the reduced average speeds on lane 1 . Higher deceleration frequency on lane 1 reduces the stability of flow on this lane. In order to obtain the highest throughput on lane 1, the frequency of action 1 on lane 1 should be minimized. Nonetheless the frequency of action 1 on lane 1 is also dependent on lane 1 and onramp demands. Therefore, in the sole operation of action 1, there is a correlation between the parameters below as the model inputs and outputs.

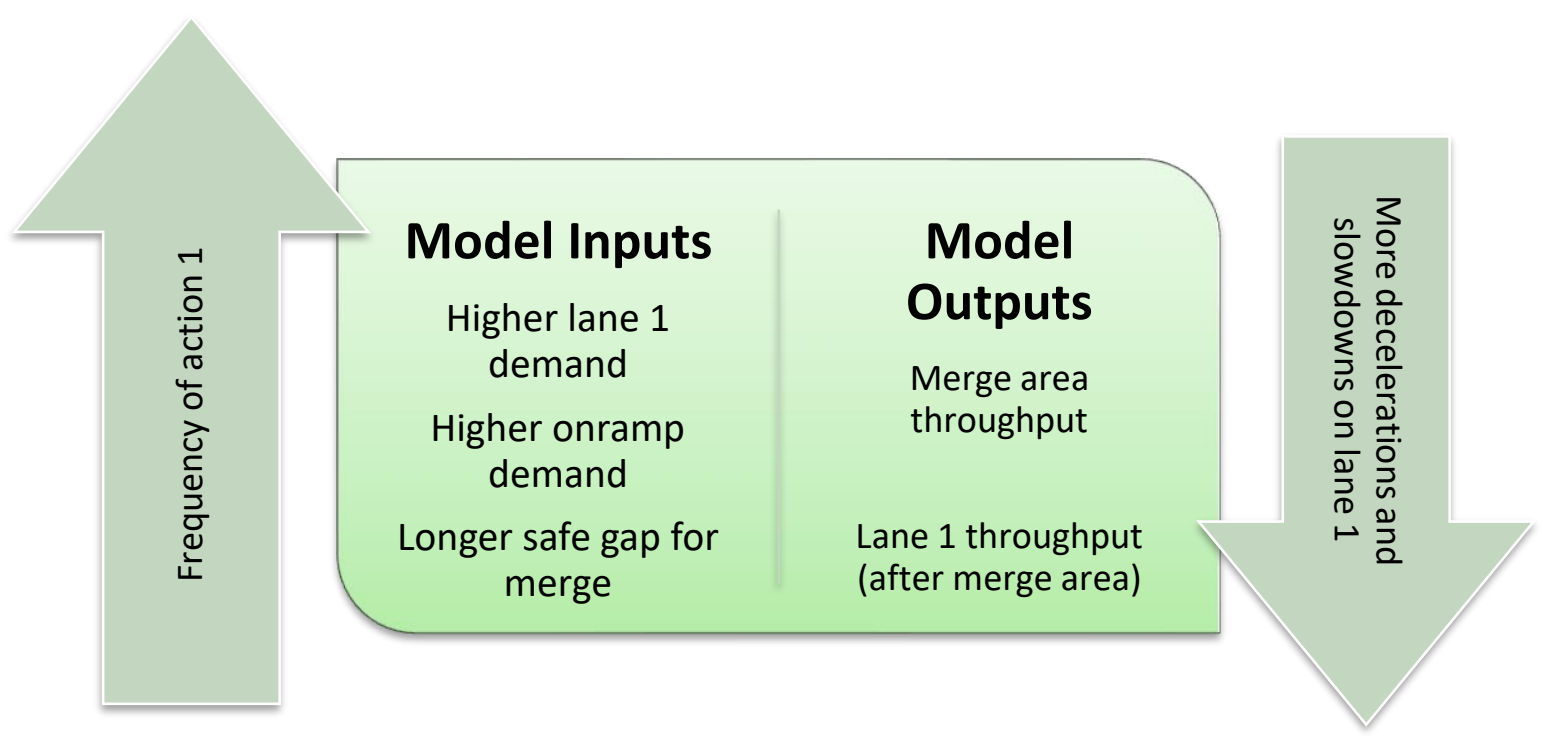

The traffic flow is considered unstable when speeds on lane 1 decline more than $5 \frac{\mathrm{m}}{\mathrm{s}}$. It is expected that the maximum throughput of lane 1 is obtained when flow on lane 1 is saturated based on the limitations of the car following model and the Rule Based model. The performance of lane 1 is captured through the traffic performance measures listed below:

1. Merge throughput

2. Lane 1 throughput 


\section{Operation of actions 1 and 2}

If action 2 is only reserved for implementation on the danger zone, then its potential would be to reduce deceleration on lane 1 and no planning before this stage would be enabled. Therefore, the operation of action 2 starts before a vehicle is on the acceleration lane. In this set of experiments, in the circumstance that a vehicle is unable to merge in the danger zone, action 1 provides the safe gap for merge.

The performance of lane 1-3 are captured through the traffic performance measures listed below:

\section{Merge throughput \\ 2. Lane changes to platoons in lane 2}

\section{Operation of actions 1-3}

For the utilization of lane 3 in the merge events, action 3 will be tested beside action 1 and 2 for the analysis of the traffic performance. The target is to yield the maximum obtainable throughput on lane 1,2 , and 3 considering the random nature of certain elements in the model. The results are expected to produce fewer action 1 through the utilization of lane change to the platoon lanes.

The throughput on lane 1 after the merge area is measured as the sum of the merged vehicles and the lane 1 traffic flow which is notated as $Q_{\max }$ (lane 1) in this research.

$$
Q_{\max (M A)}=Q_{\max (\text { lane } 1)}+\sum q_{(\text {plt })} \quad \text { Equation } 25
$$

The demand onramp flow is referred to as $q_{\text {ramp }}$ and the demand flow of lane 1 is $q_{\text {lane } 1}$. The sum of the maximum onramp and lane 1 throughputs under the given conditions, is:

$$
Q_{\max (\text { lane 1) }}=q_{\text {merge }}+q_{(1)} \quad \text { Equation } 26
$$

Lane 1 is loaded with onramp flow until it has reached maximum throughput $\left(Q_{\max (\operatorname{lane} 1)}\right)$.

$q_{\text {total }}$ is the sum of the onramp and lane 1 demand as can be seen in equation 27 :

$$
q_{\text {total }}=q_{\text {lane } 1}+q_{\text {ramp }} \quad \text { Equation } 27
$$


If the demand for lane 1 (from the onramp and lane 1 flow) is higher than $Q_{\max }$ (lane 1), as can be seen in the equation below, action 2 starts operation. Table 14 displays the boundary conditions for the operation of action 2 and 3.

$$
q_{\text {total }}>Q_{\max (\text { lane } 1) \quad \text { Equation } 28}
$$

Table 14 The thresholds for the activation of action 1-3

\begin{tabular}{|c|c|c|}
\hline Action trigger threshold & Action 1 & Action 2-3 \\
\hline Total demand of lane 1 and onramp & Lower demand & Higher demand \\
$\qquad \boldsymbol{q}_{\text {total }}$ & $q_{\text {total }}<Q_{\max (\text { lane 1) }}$ & $Q_{\max \text { (lane 1) }}<q_{\text {total }}$ \\
\hline
\end{tabular}

\section{Major questions to seek in the results?}

The simulation tests of the model will provide a framework for answering the research questions below, considering the model limitations elaborated in previous sections.

- What is the maximum throughput on lane with the Rule Based model?

- When should action 2 and 3 be activated?

- What is the maximum merge area throughput with the operation of every action?

Finally, the simulation tests provide a mapping of traffic demands to the operations of actions 1-3. The results will provide the effectiveness of each action, significance of control parameters, and the sensitivity of the relevant parameters.

The merge model of this research can be applied for the analysis of:

- Merge Area Maximum Throughput (Capacity)

- Maximum merging to mainlines (merging capacity)

- Specifications of design features

- Car following in connected-automated network 
The expected benefits of this study are a joint investigation of actions and geometry influence on the traffic performance within a connected highway comprised of platoon and non-platoon lanes. 


\section{RESULTS}

In this chapter, the results from the simulation tests of the model are presented. The results display the analysis of the relevant parameters of the model. Additionally, the results provide an indepth understanding of the significance of the parameters in the model. The design and sequence of the tests are examined in the next section.

The maximum merge area throughput can be obtained when all lanes are operating close to their maximum feasible throughput. The maximum throughput on lane 1 which has a non-platoon traffic is different from the maximum throughput of the platoon lanes. In this section the aim is to initially load lane 1 and improve the merging throughput for the onramp vehicles with the aid of action 1 . After understanding the dynamics of the model with the operation of action 1 and obtaining the best results through exhaustive search, the goal is exploiting action 2 to obtain the improved traffic performance of the model. Finally, action 3 is applied beside action 1 and 2 to complete the lane changing limitations encountered by the solo operation of action 2 .

\subsection{Experiment Design}

In order to analyze the different aspects of the merge model, initially the specific parameters influencing the merge model performance and their type are identified. The model input parameters which are deemed to be significant in the outputs are classified in three groups:
1- Geometric
2- Control Model
3- Car Following Model

Table 15 displays the parameters for each action to be tested. 
Table 15 The analyzed input parameters and the investigated effects on the outputs

\begin{tabular}{|c|c|c|}
\hline $\begin{array}{l}\text { Operating Actions } \\
\text { for the Experiment }\end{array}$ & Varying input parameter & Investigated output parameter \\
\hline \multirow{4}{*}{ Action 1} & Onramp demand & Merge and lane 1 throughput \\
\hline & Lane 1 demand & \multirow{2}{*}{$\begin{array}{ll}\text { - } & \text { Frequency of action } 1 \\
\text { - } & \text { Percentage merging with } \\
& \text { no action } \\
\text { - } & \text { Merge and lane } 1 \\
& \text { throughput }\end{array}$} \\
\hline & Minimum safe gap for merge & \\
\hline & Length of acceleration lane & $\begin{array}{l}\text { Percentage merging without } \\
\text { action } 1\end{array}$ \\
\hline \multirow[t]{2}{*}{ Action 2 and 3} & $\begin{array}{l}\text { Effect of platoon lengths on frequency } \\
\text { of actions and merge area performance }\end{array}$ & \multirow[b]{2}{*}{$\begin{array}{ll}\text { - } & \text { Frequency of actions } 1,2 \\
& \& 3 \\
\text { - } & \text { Merge area throughput }\end{array}$} \\
\hline & $\begin{array}{l}\text { Effect of intra-platoon and inter- } \\
\text { platoon headways on frequency of } \\
\text { actions and merge area performance }\end{array}$ & \\
\hline
\end{tabular}

\subsection{Analysis of action 1}

In the simulation of action 1 the goal is to determine the maximum merge area throughput (and capacity) with the operation of action 1 for creating gaps on lane 1 for merging vehicles. Lane 2 and 3 have platoon traffic which are completely isolated from lane 1 . In this setup, vehicles in lane 1 cannot join platoons to create gaps for merging vehicles. 


\subsubsection{Approach for Analysis of Action 1:}

Lane 1 is at capacity point when it breaks down. The breakdown in this research was the point when speeds of upstream vehicles declined $5 \mathrm{~m} / \mathrm{s}$ or gaps relevant to the speeds are unacceptable. The breakdown is also identified at a point when the system cannot operate due to upstream vehicles approaching the merge area vehicles at speed differences higher than $5 \mathrm{~m} / \mathrm{s}$ relative to their desired speeds.

\section{Lane One Capacity with Modified IDM+ Car Following Model}

Due to the modifications to the car following model, the highest throughput of lane 1 is expected to be higher than the capacity of a lane with the IDM+ car following model. The border conditions for lane 1 considering the modifications to the car following model are displayed in the Table 16:

For obtaining the highest throughput on lane 1, the traffic was tested with the different headways. The tests would not yield acceptable results with average headways below 1 second on lane 1 . The headway range of [0.8-1.3] seconds is the shortest headway that could function by the car following model. The maximum throughput of 3,350 vph was obtained.

The average headways less than 1 second were not tested in the model due to the reasons below:

- Yielding negative gaps in lane 1 (the car following is unable to process)

- This rate of headways on lane 1 cannot provide sufficient gaps for merging cars

- This range of headway would be uncomfortable for the occupants of the vehicles

The headway range of 1.4 -1.9 was selected for the generation of vehicles on lane 1 as the default demand input. This range maintains adequate throughput rates on lane 1 and yields conditions similar to existing traffic in highways. Additionally, this range produces gaps in the merge area which are not large enough to accommodate all the merging onramp vehicles. 


\begin{tabular}{|c|c|}
\hline Model Input & Model Output \\
\hline Lane 1 headway input range (sec) & Throughput of model $\left(\frac{v e h}{h r}\right)$ \\
\hline$[\mathbf{0 . 8 - 1 . 3 ]}$ & 3,350 \\
\hline
\end{tabular}

\subsubsection{Merge Area Capacity in HCM}

The merge area in present day traffic has lower capacity compared to the straight sections of the highway. Numerous empirical data show that the merge area capacity is $15-20$ percent lower than the uninterrupted sections of the highway, though observed capacity on highways varies significantly from day to day and from one site to the other. This lower capacity and variability have been attributed to driver behavior and variability in the types of interactions between mainline and ramp vehicles at these junctions.

According to the HCM 2016 (TRB, 2016), the capacity of basic freeway segments is a function of the free-flow speed, ranging from 2,400 passenger cars per hour per lane (pc/h/ln) for FFS (Free Flow Speed) of $75 \mathrm{mi} / \mathrm{h}$, to $2,250 \mathrm{pc} / \mathrm{h} / \mathrm{ln}$ for FFS of $55 \mathrm{mi} / \mathrm{h}$. The freeway merge segments methodology in the HCM applies the same capacity values in the analysis procedure, although research has shown that capacities at the bottleneck locations formed by the merge area are considerably lower.

\section{Base conditions for the simulations}

In every simulation, all the model inputs were inserted in a fixed range while the parameter under analysis changed from the base conditions. The base conditions can be considered the default setting for each parameter (as model input) which changes, when that parameter is being analyzed. 


\section{Default Parameter Values/Rates:}

In every simulation test one parameter is analyzed and all other parameters are fixed inputs.

Certain model inputs remain fixed in all tests of the model. These include:

- The platoon characteristics are the same for Lanes 2 and 3 at the network entrance.

- Car following model of onramp and lane 1 vehicles

- $\quad$ Merge model control steps

The base conditions were the default value of parameters in the model. The base parameters in the model are displayed in the Table 17. 
Table 17 Base settings for the simulations

\begin{tabular}{|c|c|}
\hline Parameter & Minimum Range \\
\hline Acceleration lane length (m) & $150 \mathrm{~m}$ \\
\hline Length of danger zone (m) & $70 \mathrm{~m}$ \\
\hline Headway range lane 1 (sec) & [1.4-1.9] \\
\hline Headway range onramp (sec) & {$[2.5-3]$} \\
\hline Desired speeds on lane $1(\mathrm{~m} / \mathrm{s})$ & $35-38$ \\
\hline Platoon length range (\#) & {$[2-10]$} \\
\hline Inter-platoon headway (sec) & {$[2-2.2]$} \\
\hline Intra-platoon headway (sec) & {$[0.7]$} \\
\hline Platoon speeds (m/s) & {$[37-38]$} \\
\hline Minimum safe gap for merge & $45 m$ \\
\hline
\end{tabular}

The base conditions for all the model input parameters were selected based on geometric, traffic, or general constraints of the model and the real-word conditions. The geometric parameters such as acceleration lane and length of danger zone were selected in the range available in current infrastructure. 
The headway range of vehicles in lane 1 and onramp were based on the model and real-world limitations. The platoon lengths and headways were selected according to prognostications for platoon lanes in this study. The speed of vehicles on all the lanes was set to the maximum speed that traffic can have in the current highway infrastructure. Finally, the minimum safe gap for merging was set according to the minimum safe gap introduced in the MOBIL model for IDM car following. For each test, the results were obtained by 10 rounds of simulations and obtaining the median output of the result.

\section{Test of Minimum Safe Gap required for merging}

The percentage of onramp vehicles which merge to lane 1 without any action required on lane 1 determines the effect of this parameter on the probability of an onramp vehicle to merge onto lane 1 without any deceleration on lane. In this study the minimum length for this parameter was selected from the MOBIL lane change model. The MOBIL model is adapted to each of the car following models including the IDM+ model. The MOBIL model provides the minimum safe gap for merge based on the speed of the flow which operates with the IDM+ car following model. The minimum safe gaps for merge started at $35 \mathrm{~m}$ in this analysis according to the specifications of the MOBIL model. Figure 23 displays the impact of this parameter on the frequency of action 1 . As can be seen in the Figure 23, the safe gap for merge has a direct and non-liner relation with the frequency of action 1. In the current day traffic drivers have different assumptions of the safe gap for merge, which this can be fixed to a safe length when vehicles are all connected and automated. Higher safety features will enable cars to merge in shorter distances and reduce the necessity of deceleration for gap creation on lane 1 . To consider safer minimum gaps for merge (compared to the length proposed by MOBIL) and for a better analysis of the action frequency, the minimum safe gap in the next sections was increased to $45 \mathrm{~m}$. 


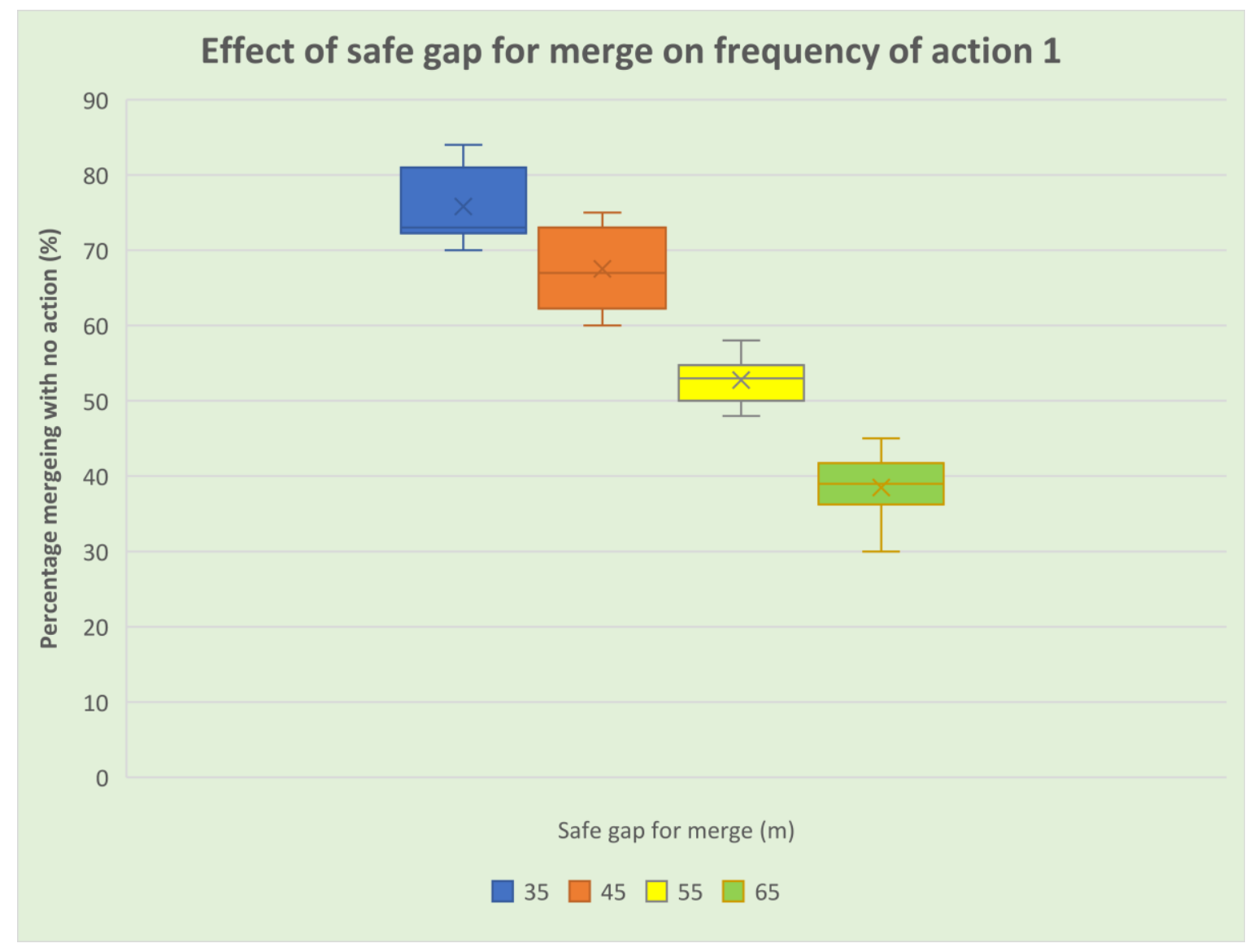

Figure 23 The effect of safe gap for merge on the frequency of action 1

\section{Relation of Average Travel Time in lane 1 and actions}

It is important to seek the correlation between frequency of actions and the travel time. Because of the decelerations on lane 1 from action 1, the average travel times on this lane can be higher than uninterrupted sections. The average travel time is influenced by the frequency of action 1 on lane 1 . Table 18 depicts the travel times with different occurrence rates of action 1 . As can be seen, with the increment of safe gap from for merging from $45 \mathrm{~m}$ to $60 \mathrm{~m}$, the average travel times increase by $4 \%$. This shows the low impact of the merging actions on the travel times within the merge area. The reason for this low impact is that the time headways between vehicles on lane 1 is within a range that the deceleration actions occurring due to the actions do not propagate to a large distance behind the decelerating vehicle. 
Table 18 The effect of minimum safe gap for merge on merge area throughput

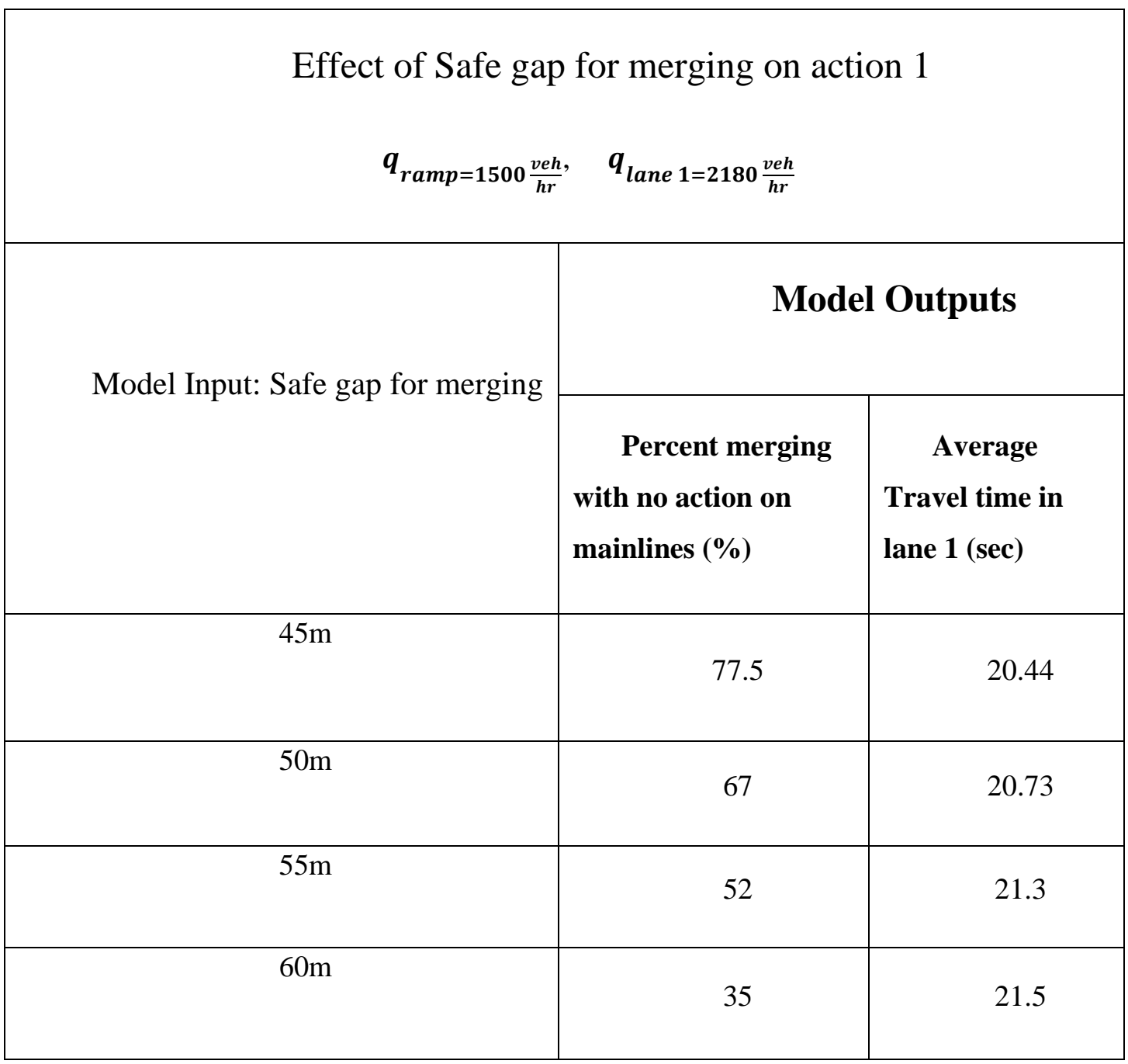

\section{Effect of Increasing Onramp Demand on Lane 1 Throughput}

As mentioned in the previous chapter, to prevent the breakdown of the merge model, the onramp demand must be equal or less than lane 1 demand. In this test, the performance of the merge area with different onramp demands were evaluated while the lane 1 demand is constant at $2180 \mathrm{vph}$. This rate for the demand on lane 1 was set to replicate the approximate capacity of lanes on current day traffic and observe the maximum merging volume added to this lane.

The table below displays the effects of increasing onramp demand- while the demand on lane 1 is constant - on the throughput of the merge area. As can be seen in this table, the maximum throughout 
obtained in lane 1 after the merge area is approximately $3500 \frac{\mathrm{veh}}{\mathrm{hr}}$. In order to test the maximum onramp demand on the lane 1 throughput (while maintaining the lane 1 demand constant), tests with increasing onramp demand were implemented.

displays the change of merge area throughputs with the increasing onramp demand.

The results of the simulation show that:

- With a lane 1 flow of $2180 \frac{v e h}{h r}$, the traffic on lane 1 became unstable when the demand of onramp vehicles exceeded $1756 \frac{\mathrm{veh}}{\mathrm{hr}}$

- The maximum lane 1 throughput in the merge area of the model was $3475 \frac{\mathrm{veh}}{\mathrm{hr}}$

These rates display the maximum throughput on lane 1 for the merge area considering the capability of the car following model and the maximum merging allowance. The breakdown conditions displayed in Table 19 were the conditions when the model was unable to correctly process the vehicles. This outcome was visible with negative gaps and speed drops of more than $5 \mathrm{~m} / \mathrm{s}$ on lane 1.

Table 19 Effect of increasing onramp demand on lane 1 throughput (lane 1 demand is set to 2,180 vph, safe gap for merge $=45 \mathrm{~m}$ )

\begin{tabular}{|c|c|c|c|}
\hline \multicolumn{2}{|c|}{ Input } & \multicolumn{2}{c|}{ Output: Lane 1 Throughput (vph) } \\
\hline $\begin{array}{c}\text { Onramp Demand } \\
(\mathrm{vph})\end{array}$ & Headway range (s) & Range & Average \\
\hline 1,220 & {$[2.8-3.1]$} & $3150-3200$ & 3,175 \\
\hline 1,359 & {$[2.5-2.8]$} & $3280-3320$ & 3,300 \\
\hline 1,530 & {$[2.2-2.5]$} & $3450-3500$ & 3,475 \\
\hline 1,756 & {$[1.9-2.2]$} & Breakdown & Breakdown \\
\hline
\end{tabular}

The throughputs obtained are less than the overall demands, as some vehicles did not finish the simulation length to be recorded in the throughput. 
To investigate the simultaneous effect of increasing onramp and lane 1 demand, the simulation tests were implemented to measure the lane 1 merge area throughput. The onramp flow was increased from $900 \frac{v e h}{h r}$ to $1350 \frac{v e h}{h r}$ while the lane 1 demand was increased from $1310 \frac{v e h}{h r}$ to $2050 \frac{v e h}{h r}$. The results indicate that for action 1, the maximum throughput on lane 1 without system breakdown was approximately $3350 \frac{\mathrm{veh}}{\mathrm{hr}}$. This rate is close to the capacity of lane obtained in the previous section. Table 20 and Figure 24 display the results of this section's simulations.

Table 20 Analysis of the simultaneous effect of increasing onramp and lane 1 demand on the merge area throughput

Throughput on lane 1 (vph) downstream the onramp merge

\begin{tabular}{|c|r|r|r|r|}
\hline \multirow{2}{*}{$\begin{array}{c}\text { Model Inputs } \\
\text { Lane 1 flow } \\
\text { (veh/hr) }\end{array}$} & \multicolumn{4}{|c|}{ Onramp flow demand (vph) } \\
\cline { 2 - 5 } & 900 & 1,050 & 1,200 & 1,350 \\
\hline 1,310 & 2,100 & 2,250 & 2,400 & 2,640 \\
\hline 1,600 & 2,400 & 2,500 & 2,660 & 2,900 \\
\hline 2,050 & 2,826 & 2,952 & 3,100 & 3,340 \\
\hline
\end{tabular}




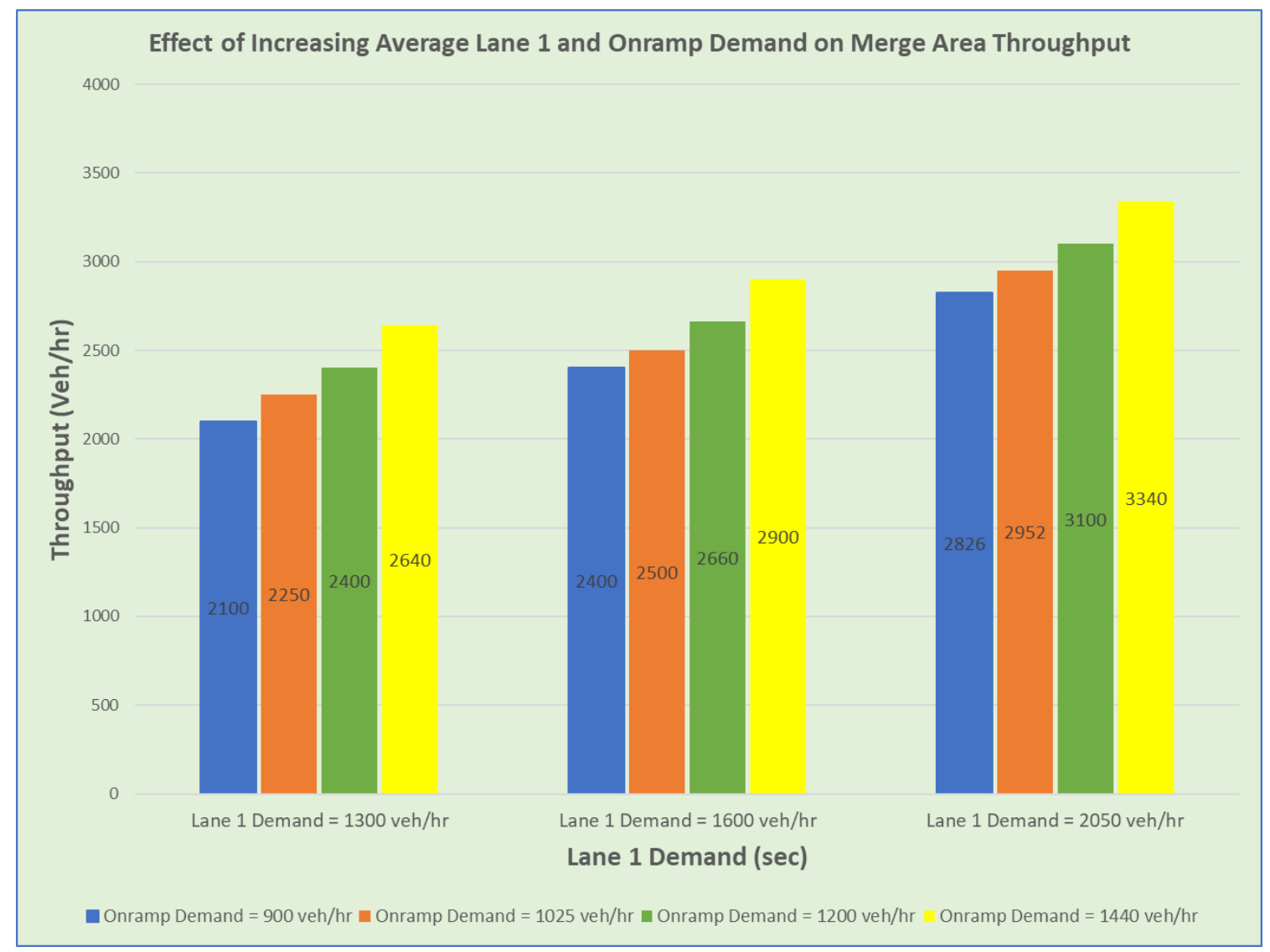

Figure 24 simultaneous effect of increasing onramp and lane 1 demand on the merge area throughput

\section{Analysis of the effect of headways on lane 1 on the merge area throughput}

In this test the aim was to understand how changes in the headways of vehicles generated on lane 1 impacts the frequency of action 1 . For the analysis of the headways on lane 1 , two headway ranges were tested. The headways were selected in a range that were appropriate for the lane 1 car following and the merge model. For the functionality of the model, onramp headway range have to be larger than the headways on lane 1 and thus in testing the longer headways (2.2-2.6 seconds) on lane 1, the onramp range of headways had to be increased to the minimum possible for processing which was (3.5-4) seconds. Additionally, the detection zone for the onramp vehicles were modified according to the average headways on lane 1.

The results displayed on Table 21 and Figure 25 showed that longer headway ranges on lane 1 reduce the throughput, even though the number of actions on lane 1 decline. This shows the larger impact of demand compared to the frequency of actions on lane 1 throughput. When the headways on 
lane 1 were increased to the range of 2.2-2.6 seconds, in the vehicle generation of lane 1 , the vehicles are all generated having gaps larger than the safe gap required for merge, but by the time they pass the merge area, due to the different desired speeds that they have, some gaps become shorter than the minimum safe gap required for merge and thus action 1 still occurs.

Table 21 Effect of headways on lane 1 on the frequency of action 1 and merge area throughput

\begin{tabular}{|c|c|c|c|c|}
\hline \multirow{3}{*}{$\begin{array}{l}\text { Model Input } \\
\text { Headway } \\
\text { range on lane } 1 \\
\text { (sec) }\end{array}$} & \multicolumn{2}{|c|}{$\begin{array}{c}\text { Input: Safe gap for merge }= \\
\qquad 50 \mathrm{~m}\end{array}$} & \multicolumn{2}{|c|}{$\begin{array}{l}\text { Input: Safe gap for merge }= \\
\qquad 55 \mathrm{~m}\end{array}$} \\
\hline & \multicolumn{2}{|c|}{ Output lane 1} & \multicolumn{2}{|c|}{ Output lane 1} \\
\hline & \begin{tabular}{l}
\multicolumn{1}{c}{ Lane } \\
One \\
Throughput \\
(veh/hr)
\end{tabular} & $\begin{array}{c}\text { Average } \\
\text { merged with no } \\
\text { action }(\%)\end{array}$ & $\begin{array}{l}\text { Lane One } \\
\text { Throughput } \\
\text { (veh/hr) }\end{array}$ & $\begin{array}{l}\text { Average merged } \\
\text { with no action }(\%)\end{array}$ \\
\hline$[1.4-1.9]$ & 2,650 & 94 & 2,676 & 89 \\
\hline$[2.2-2.7]$ & 2,431 & 100 & 2,375 & 100 \\
\hline
\end{tabular}

The results above display that headways above 2.2 second on lane 1 , with the inserted demand volumes, prevent the occurrence of actions. On the other hand, average headways of 2 seconds on lane one reduces the throughput of this lane, compared to the headway ranges shorter than 2 seconds. 


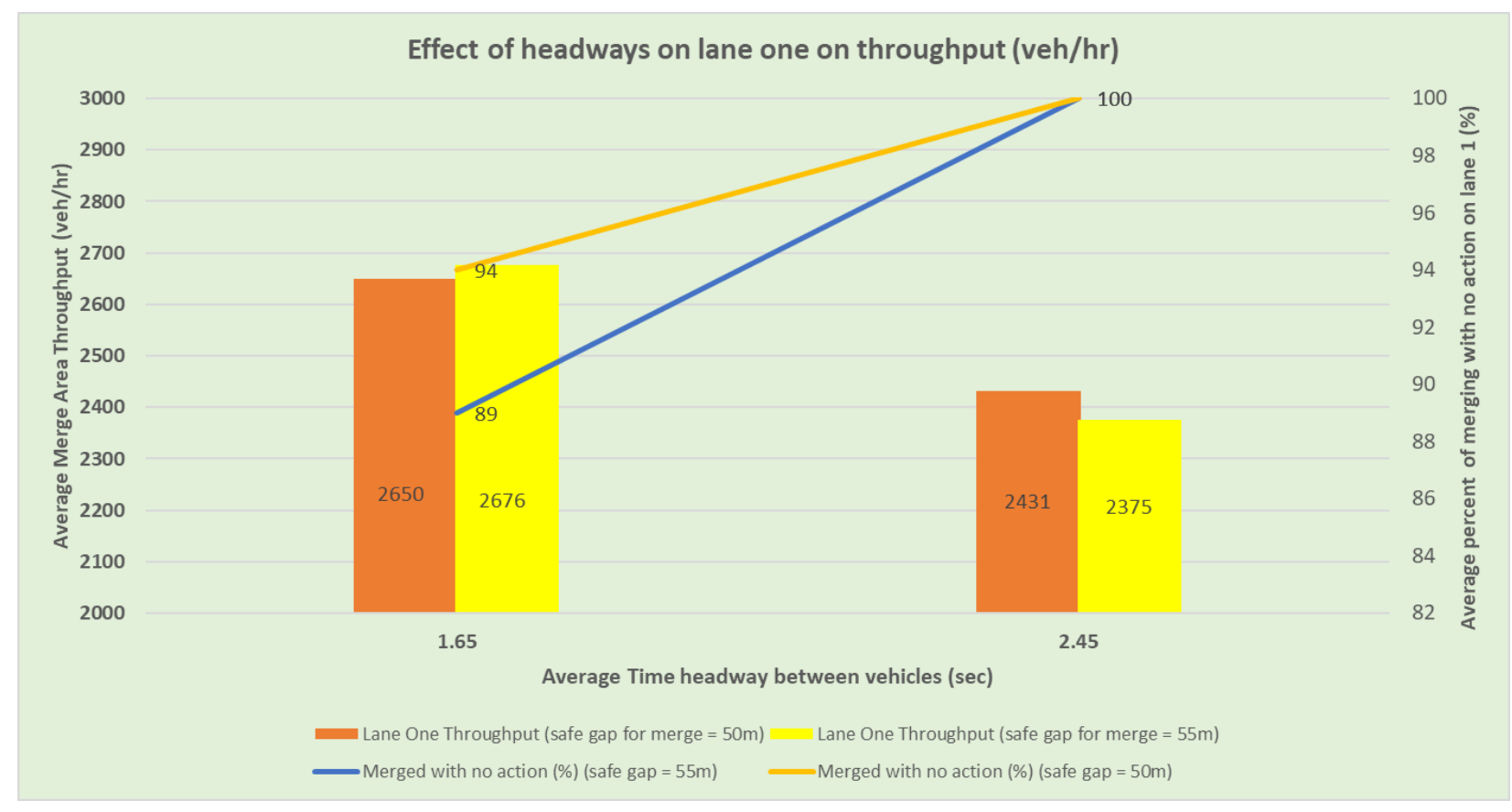

Figure 25 Effect of headways on lane 1 on merge area throughput

\subsubsection{Effect of Geometry on Action Prevention}

One of the geometric factors influencing the merge area which affects the onramp merge capacity and the merge area throughput is the length of the acceleration lane. The acceleration lane for the merging vehicles is parallel to the mainlines. It can be expected that the longer these lanes, the more opportunity for the onramp vehicles to find a suitable merging gap without a necessary action on lane 1. On the other hand, geometric and topographic constraints also limit the length of a speeding lane in real world highways.

The length of the acceleration lane can have a big impact on the merge completion. Longer acceleration lanes provide longer time for drivers to observe the traffic on lane 1 and find a suitable gap for merge. Additionally, longer acceleration lanes can enhance safety of the merge by eliminating the enforcement of the merge when lane 1 traffic has high speeds and volumes.

In this section, simulations were executed to understand the effect of the acceleration lane on preventing action 1 on lane 1. In the test results displayed in and Figure 27-27 and Table 22, while 
maintaining uniform simulation parameters, different acceleration lanes were tested to determine the effect on the percentage of onramp vehicles merging with no action.

Table 22 Analysis of the length of acceleration lane on the frequency of action 1

\begin{tabular}{|c|l|l|}
\hline $\begin{array}{c}\text { Acceleration } \\
\text { lane length, } L_{\text {accl }}\end{array}$ & $\begin{array}{c}\text { Percentage of } \\
\text { onramp vehicles } \\
\text { merging with no action } \\
(\%)\end{array}$ & $\begin{array}{l}\text { Median percentage of } \\
\text { onramp vehicles merging with } \\
\text { no action (\%) }\end{array}$ \\
\hline $150 \mathrm{~m}$ & $85-90$ & 86 \\
\hline $200 \mathrm{~m}$ & $88-93$ & 90 \\
\hline $250 \mathrm{~m}$ & $90-95$ & 91.5 \\
\hline
\end{tabular}

The results of the simulation above show that increasing the length of the acceleration lane can reduce the number of actions on lane 1 . Subsequently less actions on lane 1 leads to lower delays by action 1 which can increase throughput on this lane within a given period.

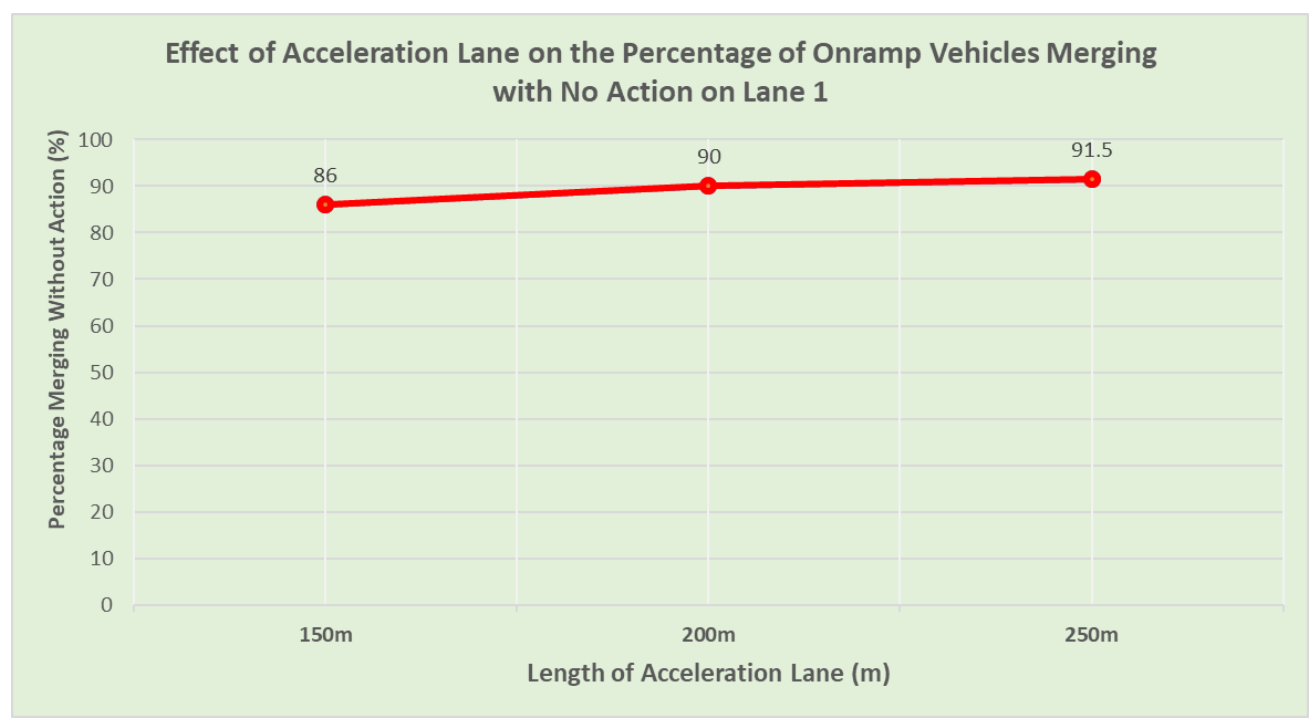

Figure 26 Effect of acceleration lane on the frequency of action 1 


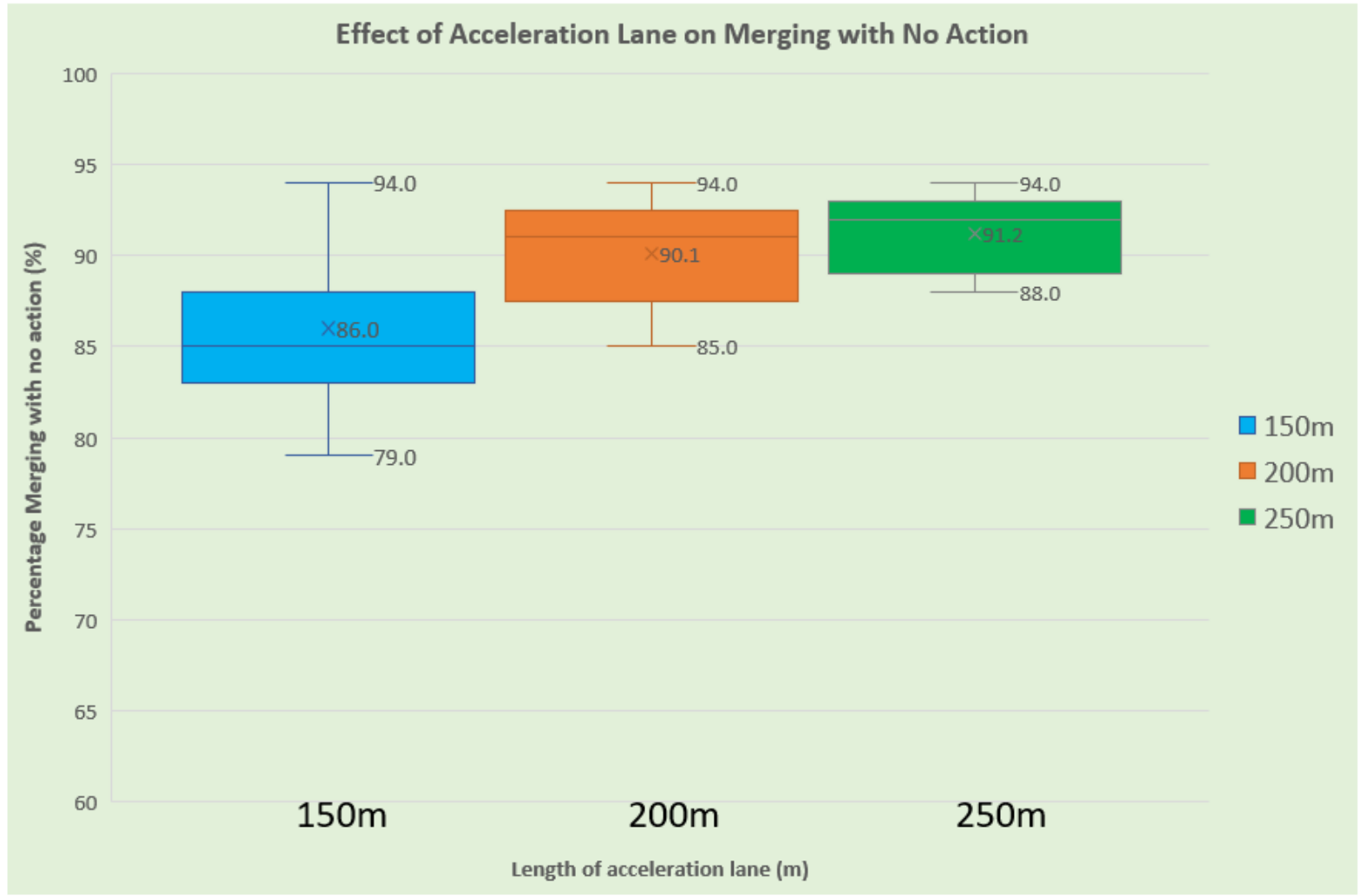

Figure 27 Effect of acceleration lane on the frequency of action 1

\subsection{Simulation of action 2}

In this section the simulation tests for the analysis of the model with the operation of actions 1-2 were implemented. The model was evaluated with the operation of actions 1 and 2 to determine the performance and enable comparison between actions.

The simulation tests below were investigated to obtain the effects of action 2 on the merge area throughput.

- Analysis of increasing platoon lengths of lane 2 on the frequency of actions 1 and 2

- Analysis of platoon length on percentage of onramp vehicles merging without action 1

- Analysis of inter-platoon headway on frequency of action 2 


\section{Effect of increasing platoon lengths of lane 2 on the number of actions 1 and 2}

The aim of this test is to understand how increasing platoon lengths can affect the frequency of actions 2 which take place in a specific time period. Action 2 completion requires the possibility for a vehicle to join a platoon in the adjacent lane and thus platoon length can influence the feasibility of action 2. Table 23and Figure 28 display the frequency of actions 2 in simulation with varying platoon lengths.

The results show that higher platoon lengths reduce the frequency of action 2 . This result can be related to the conditions considered for the vehicles joining the end or front of a platoon, nonetheless the merge area throughput increases with longer platoon lengths. It is understood that longer platoons, increase the throughput of the platoon lane, but reduce the possibility of action 2. Lower action 2 frequencies provide fewer lane changes for gap creation on lane 1. In the next section the relation between occurrence of action 1 and 2 is presented. Table 23 and Figure 28 display the frequency of action 2 relative to the platoon lengths. The frequency displayed showed that approximately $20 \%$ of merging vehicles succeeded to merge with the gap created by action 2 (onramp demand $=1200 \mathrm{vph}$ ).

Table 23 Analysis of platoon length on the merge area throughput

\begin{tabular}{|c|c|}
\hline Model Inputs & Model Output \\
\hline $\begin{array}{c}\text { Effect of Platoon length on } \\
\text { merge area throughput (1hr) } \\
\text { Onramp demand =1,280 vph }\end{array}$ & $\begin{array}{c}\text { Median number of actions 2 } \\
(\mathbf{h r})\end{array}$ \\
\hline$[2-5]$ & 346 \\
\hline$[5-8]$ & 306 \\
\hline$[8-11]$ & 255 \\
\hline
\end{tabular}




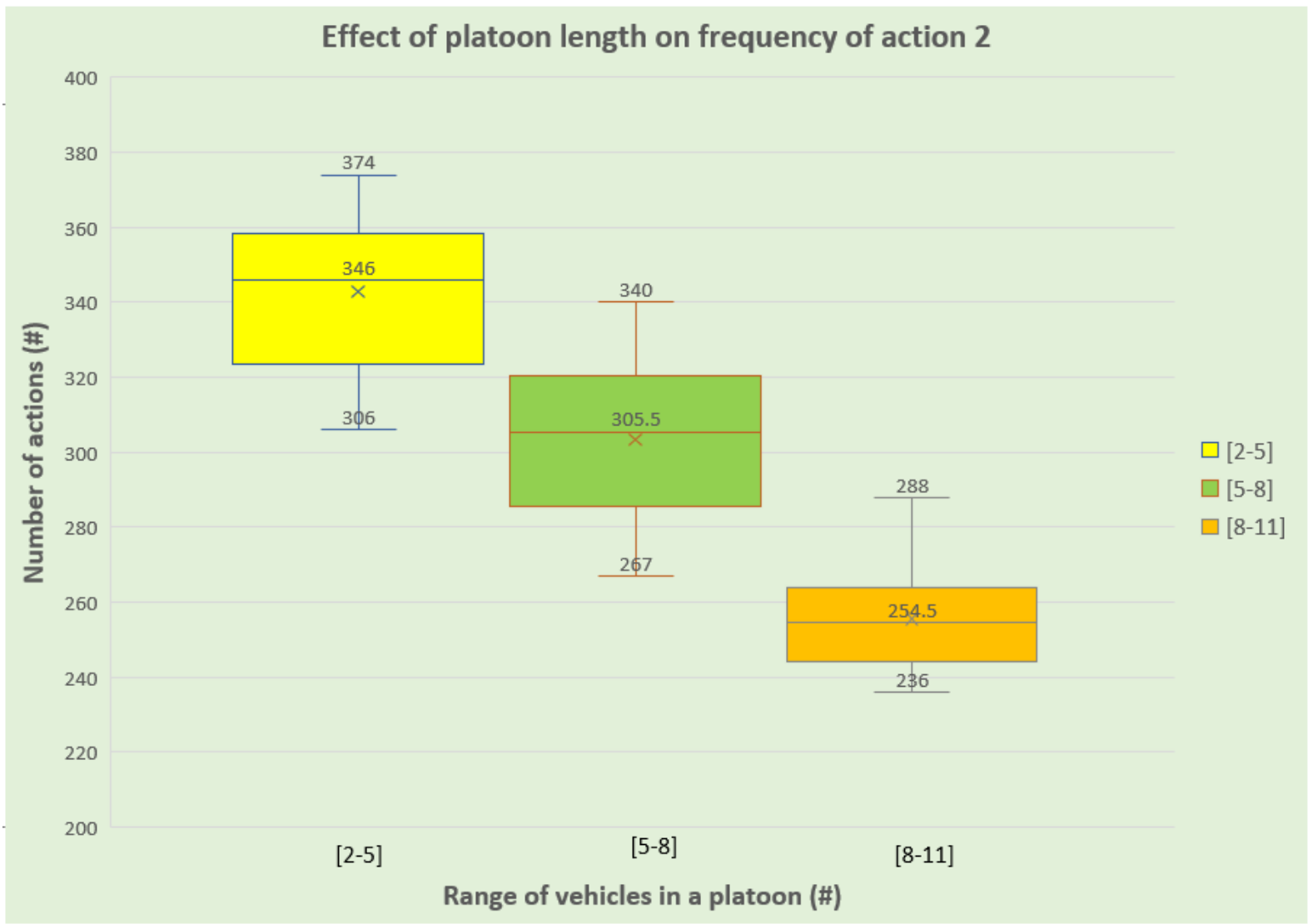

Figure 28 Analysis of the relations between platoon length and number of actions 2

\section{Effect of platoon length on percentage merging with no action 1}

In the previous section the results showed that higher platoon lengths can reduce the frequency of action 2. In this section simulations were executed to evaluate the effect of longer platoons on the frequency of action 1. Table 24 and Figure 29 display the results of this analysis. The results show that increasing platoon length slightly increases the frequency of action 1, while reducing the frequency of action 2. Lower rates of action 2, create fewer safe gaps on lane 1 , which subsequently leads to more action 1 required for gap creation. 
Table 24 Effect of platoon length on percentage merging with no action 1

\begin{tabular}{|c|c|c|}
\hline $\begin{array}{c}|c| \\
\text { Model Input } \\
\text { Onramp demand = } \\
\text { 1280 vph }\end{array}$ & \multicolumn{2}{|c|}{ Model Output } \\
\hline Platoon length range & Average number of action 2 & Average number of action 1 \\
\hline$[2-5]$ & 395 & 102 \\
\hline$[5-8]$ & 304 & 146 \\
\hline$[8-11]$ & 192 & 196 \\
\hline
\end{tabular}

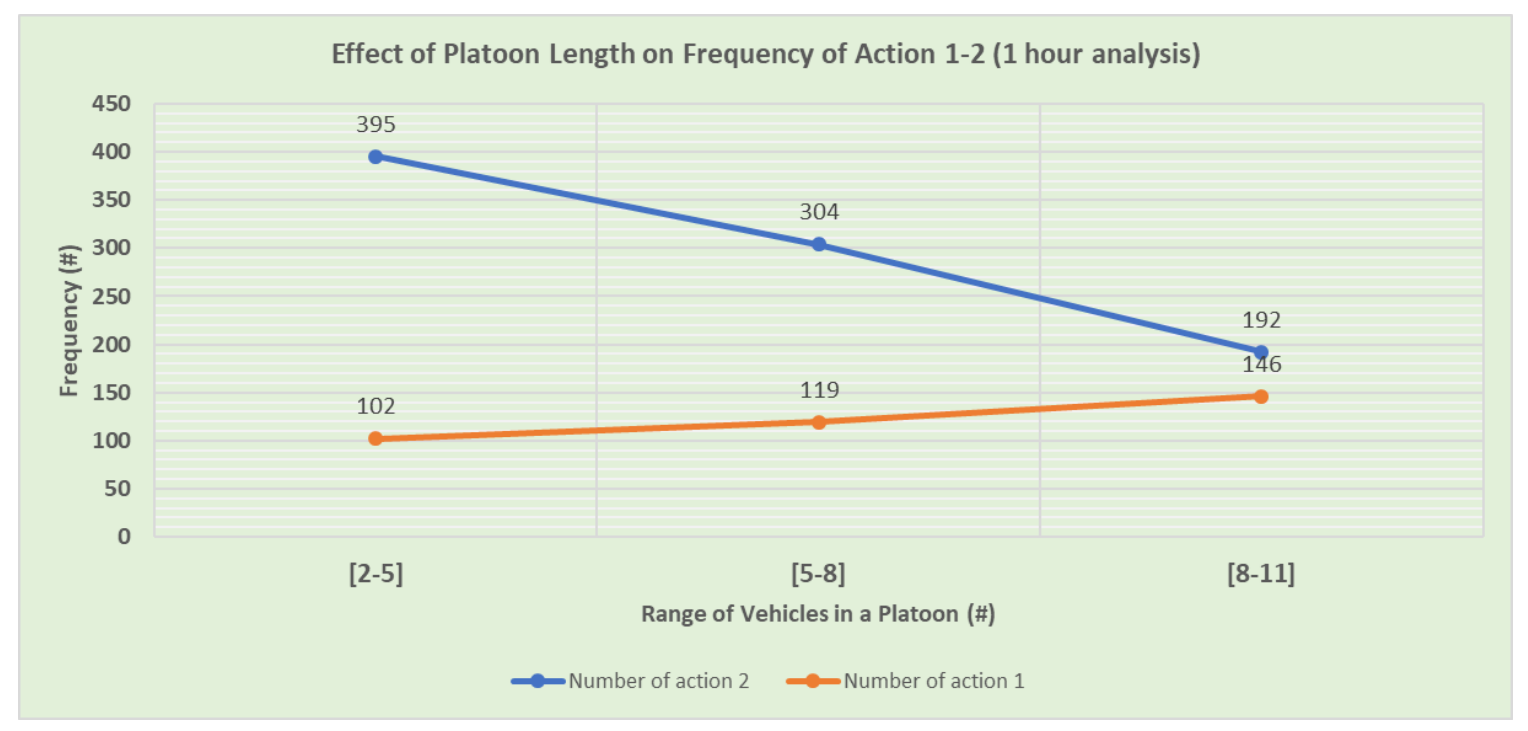

Figure 29 Effect of platoon length on percentage merging with no action 1

\section{Effect of inter-platoon headway on reduction of action 2}

The aim of this section is to analyze how increasing inter-platoon headways can affect the frequency of actions 2 which take place in a specific time period. Action 2 completion requires the 
possibility for a vehicle to join a platoon in the adjacent lane and thus inter-platoon headway determines the space between consecutive platoons. From a statistical analysis, it can be understood that longer inter-platoon headways can create a higher probability for a vehicle to join from lane 1. Table 25 and Figure 30 display the frequency of actions 2 in simulation with different inter-platoon headways. The results show that higher inter-platoon headways reduce the frequency of action 1 . The frequency of action 2 does not have a direct correlation with inter-platoon headway. The simulation results cannot suggest a specific inter-platoon headway for the highest frequency of action 2 .

Table 25 Analysis of inter-platoon headway reduction on frequency of actions 2

\begin{tabular}{|c|c|c|}
\hline Model Inputs & \multicolumn{2}{|c|}{ Model Outputs } \\
\hline $\begin{array}{l}\text { Effect of inter-platoon headway } \\
\text { reduction on number of actions } 2\end{array}$ & \multirow[t]{2}{*}{$\begin{array}{l}\text { Average frequency } \\
\text { of action } 1\end{array}$} & \multirow[t]{2}{*}{$\begin{array}{l}\text { Average frequency } \\
\text { of action } 2\end{array}$} \\
\hline Interplatoon headway range (sec) & & \\
\hline$[1.8-2]$ & 153 & 118 \\
\hline$[2-2.2]$ & 144 & 126 \\
\hline$[2.2-2.4]$ & 122 & 139 \\
\hline$[3-3.5]$ & 64 & 156 \\
\hline
\end{tabular}




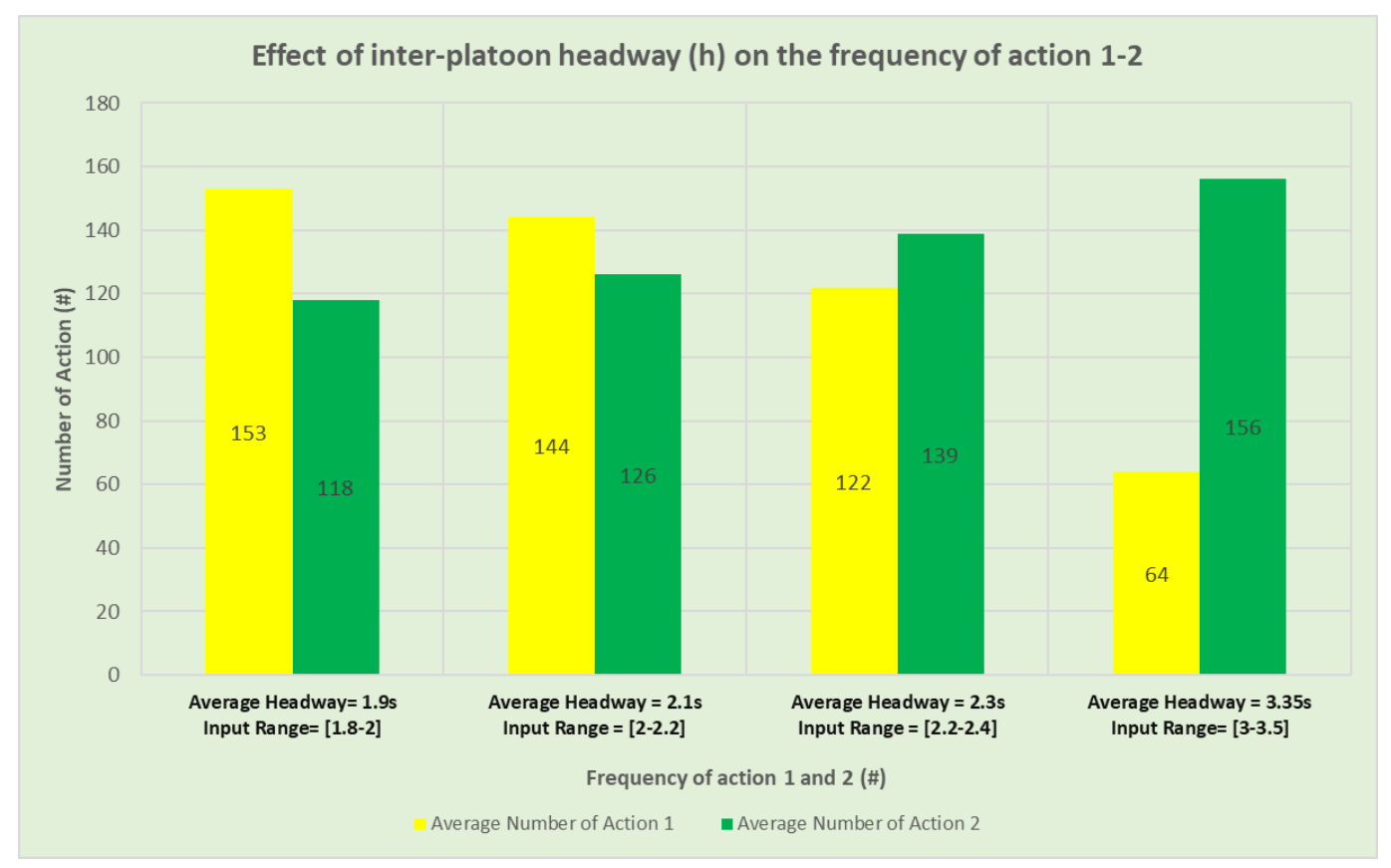

Figure 30 Analysis of inter-platoon headway reduction on reducing number of action 1 and 2 


\subsection{Simulation of action 3}

Addition of the possibility for vehicles in lane 2 to join platoons in lane 3 can add to the merge area throughput by utilizing the potential of lane 3 . Subsequently the potential additional throughput that lane 2 and 3 offer is enabled. Action 3 is triggered to incorporate this feature via the sensor relaying the information of every onramp vehicle while it still is in the onramp and enroute to enter the acceleration lane.

The operation of action 3 in this model provides the utility of the 3 lanes considering the constraints and limitations of the model. Action 3 can create gaps on lane 1 by the required lane changes on lane 2 and 3 and reduce the frequency of action 1. Furthermore action 3 can increase the platoon traffic flow and the throughput (efficiency) of platoon lane.

\section{Action 3 settings and prerequisites}

- Onramp vehicle detection prior to the vehicle entering the acceleration lane

- Lane change compliance (from lane 1 to 2)

- Lane change not feasible from lane 1 to 2 unless action 3 takes place

Similar to the analysis of action 2 , for the analysis of action 3 , the analysis listed below is conducted:

- Analysis of increasing platoon lengths on the number of actions 1-3

- Analysis of platoon length on percentage of onramp vehicles merging without action 1

- Analysis of inter-platoon headway on frequency of action 3

\section{Effect of platoon length on frequency of actions 3}

To evaluate the effect of longer platoons on the frequency of action 1, simulations tests were executed with different platoon lengths. The Table below displays the results of this analysis. The results show that increasing platoon length slightly increases the frequency of actions 1, while reducing the frequency of actions 2 . If we consider two platoons and their inter-platoon headway as one entity, then, with longer platoons and constant inter-platoon headway, the probability of a platoon on lane 2 being beside the gap on lane 2 declines. The image below displays a comparison of the inter-platoon gap availability gaps reduction between 2 platoon lanes with different platoon lengths. 
As can be displayed in the Figure 31, the platoon lane with 5 vehicles provides fewer inter-platoon gaps in the same length of the highway when compared to the platoon lane with 3 vehicles.

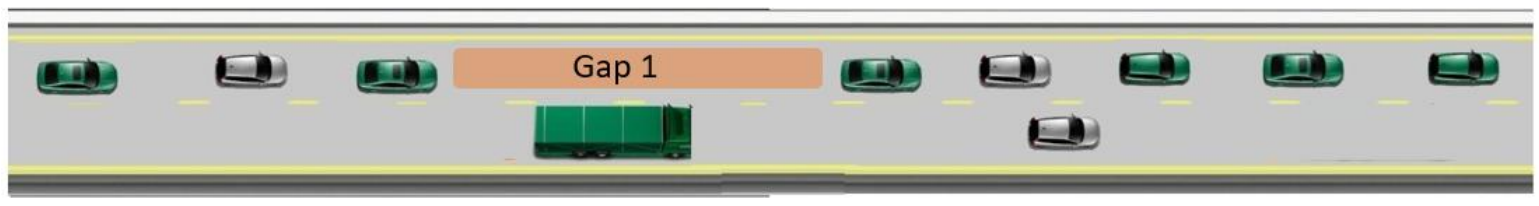

Left lane with 5 vehicles in each platoon

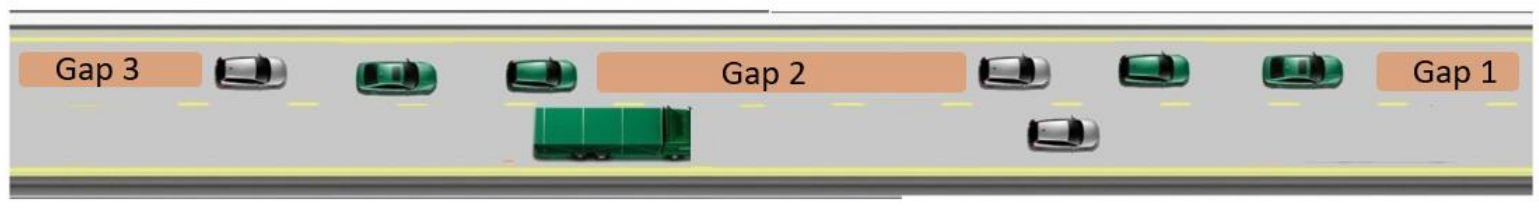

Left lane with 3 vehicles in each platoon

Figure 31 The effect of longer platoon lengths on the inter-platoon gap

\section{Access to inter-platoon gaps}

The formation of two platoons provides one inter-platoon gap between them. Figure 32 displays such formation. This formation has the length, $\left(h+2 L_{p l t}\right)$. In the figure below, the ratio of the inter-platoon gap to the whole length is:

The ratio of the inter-platoon gap to the whole length $=\frac{h}{h+2 L_{p l t}}$

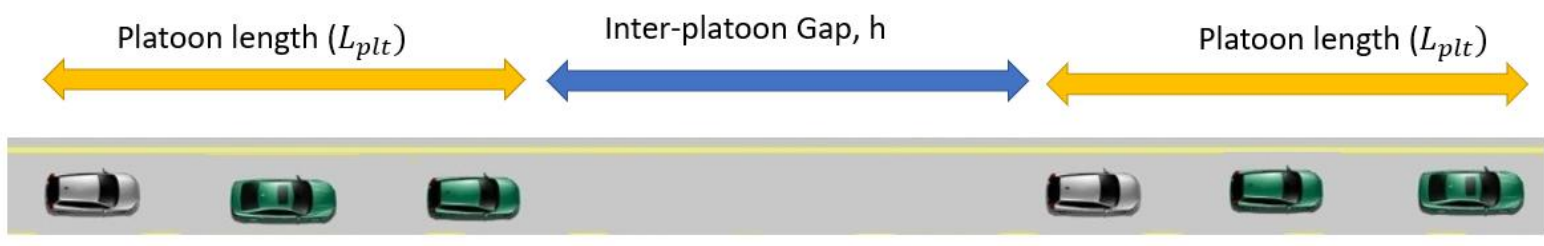

Figure 32 The access to inter-platoon gaps relative to the length of two consecutive platoons

Assuming a constant length of $5 \mathrm{~m}$ for cars, $80 \mathrm{~m}$ for the inter-platoon gap and $25 \mathrm{~m}$ for the intra-platoon gap, Table 26 displays the ratio of the inter-platoon gap in between two consecutive platoons with different lengths. 
Table 26 Ratio of the inter-platoon gap in the between two consecutive platoons

\begin{tabular}{|c|c|}
$\begin{array}{c}\text { Ratio of inter-platoon } \\
\text { gap to the total length of } 2 \\
\text { platoons }\end{array}$ & $\begin{array}{c}\text { Ratio of the inter- } \\
\text { platoon gap to the length } \\
\text { of } 2 \text { platoons }\left(\frac{h}{h+2 L}\right)\end{array}$ \\
\hline $\begin{array}{c}\text { Number of Vehicles in a } \\
\text { Platoon (\#) }\end{array}$ & \\
\hline $\mathbf{3}$ & $0.38 \mathrm{~m}, \boldsymbol{i}=\mathbf{2 5 m}$ \\
\hline $\mathbf{6}$ & 0.20 \\
\hline $\mathbf{9}$ & \\
\hline
\end{tabular}

The results of the analysis of platoon length on the number of actions 3 are displayed on Table 27 and Figure 33. Longer platoons create less possibility for the feasibility of action 3. Lower rates of action 2-3, creates fewer safe gaps on lane 1, which subsequently leads to more action 1 for gap creation. The occurrence of action 1 is related to a percentage of onramp vehicles which an action 2 or 3 was not executed for their merge. The results also show that with increasing platoon length the percentage of actions 2 leading to action 3 decline. 
Table 27 Analysis of platoon length on number of actions 1- 3

\begin{tabular}{|c|c|c|c|c|}
\hline Model Inputs & \multicolumn{4}{|c|}{ Model Outputs (One-hour simulation) } \\
\hline $\begin{array}{c}\text { Range of } \\
\text { Platoon Length in } \\
\text { Vehicles (n) }\end{array}$ & $\begin{array}{c}\text { Average } \\
\text { number of } \\
\text { action 3 }\end{array}$ & $\begin{array}{c}\text { Average } \\
\text { action 2 }\end{array}$ & $\begin{array}{c}\text { Average } \\
\text { number of } \\
\text { action 1 }\end{array}$ & $\begin{array}{c}\text { Number of } \\
\text { merged vehicles } \\
\text { (vph) }\end{array}$ \\
\hline$[2-5]$ & 91 & 156 & 25 & 1224 \\
\hline$[5-8]$ & 60 & 109 & 86 & 1220 \\
\hline$[8-11]$ & 22 & 48 & 102 & 1220 \\
\hline
\end{tabular}

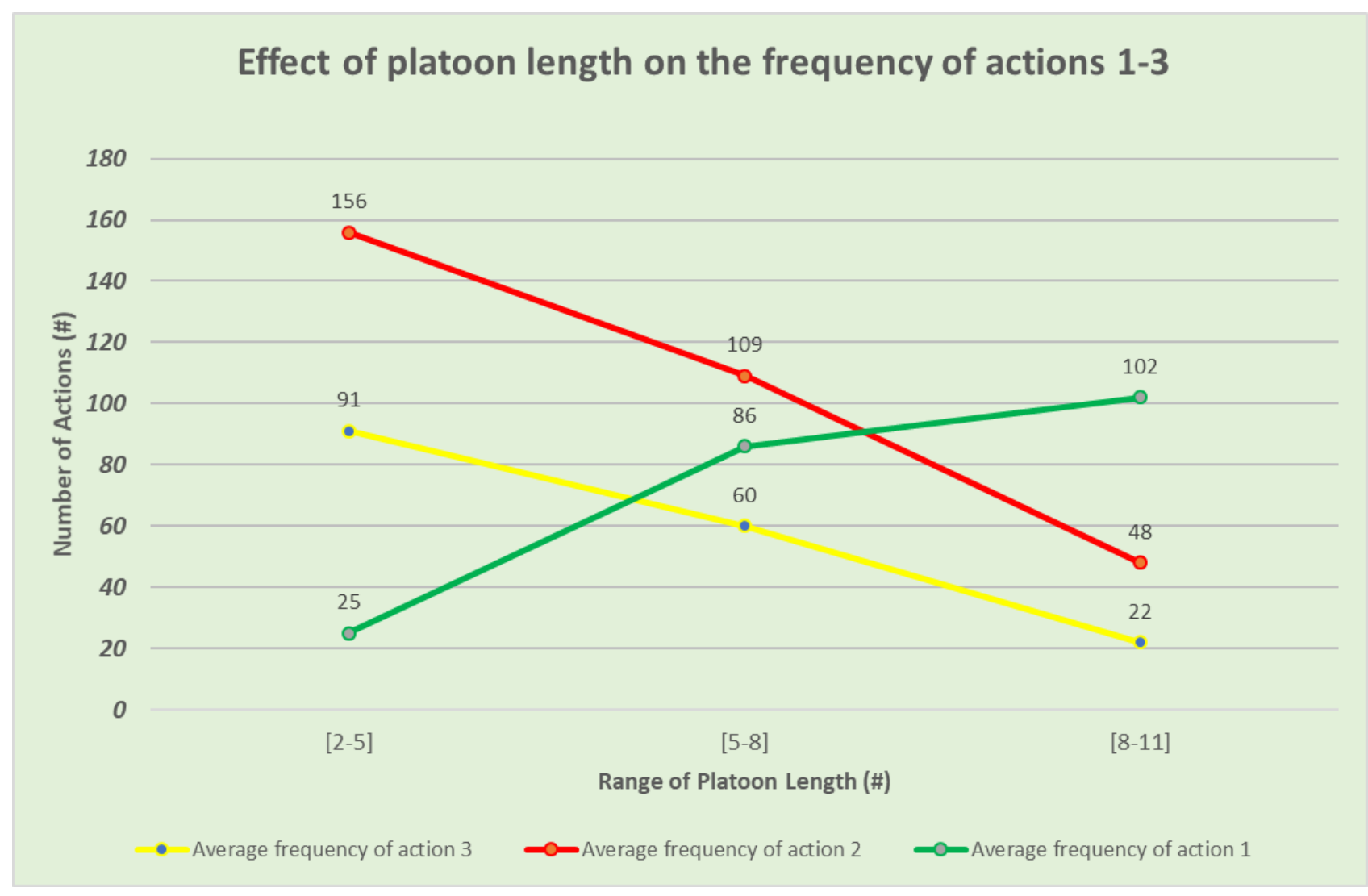

Figure 33 Effect of platoon length on the merge area throughput and number of actions 3

Analysis of the effects of inter-platoon headway on number of actions 3 
The aim of this section is to analyze how increasing inter-platoon headways can affect the frequency of actions 3 which take place in a specific time period. Action 3 completion requires the possibility for a vehicle to join a platoon in the adjacent lane and thus inter-platoon headway determines the space between consecutive platoons.

From a statistical analysis, it can be expected that with a constant average platoon length, longer inter-platoon headways can create a higher probability for a vehicle to join the platoons. Table 28 and Figure 34 display the frequency of actions 3 in simulation with varying inter-platoon headways in a five-minute analysis. The test was implemented with platoon lengths ranging from 3-7 vehicles. The results show that higher inter-platoon headways reduce the number of actions 3 . With an inter-platoon headway in the range of [2-2.2] seconds, the highest number of actions 2 occur, thus showing the effectiveness of this range. Different inter-platoon headways do not have a significant effect on the frequency of action 3. From the results, it can be understood that the frequency of inter-platoon headway is more important than the length of the headway for the occurrence of action 3 . This can be explained by the thresholds defined for the operation of action 3 .

Table 28 Analysis of the effects of inter-platoon headway on number of action 1-3 in a 5 minute simulation period

\begin{tabular}{|c|c|c|c|}
\hline Model Inputs & \multicolumn{3}{|c|}{ Model Outputs (5 min analysis) } \\
\hline $\begin{array}{l}\text { Inter-platoon } \\
\text { headway (sec) }\end{array}$ & $\begin{array}{l}\text { Average } \\
\text { number of } \\
\text { action } 3\end{array}$ & $\begin{array}{c}\text { Average } \\
\text { number of } \\
\text { action } 2\end{array}$ & $\begin{array}{r}\text { Average } \\
\text { number of } \\
\text { action } 1\end{array}$ \\
\hline$[1.8-2]$ & 6 & 42 & 5 \\
\hline$[2-2.2]$ & 6 & 58 & 12 \\
\hline$[2.2-2.4]$ & 6 & 48 & 11 \\
\hline$[3-3.5]$ & 7 & 46 & 11 \\
\hline
\end{tabular}




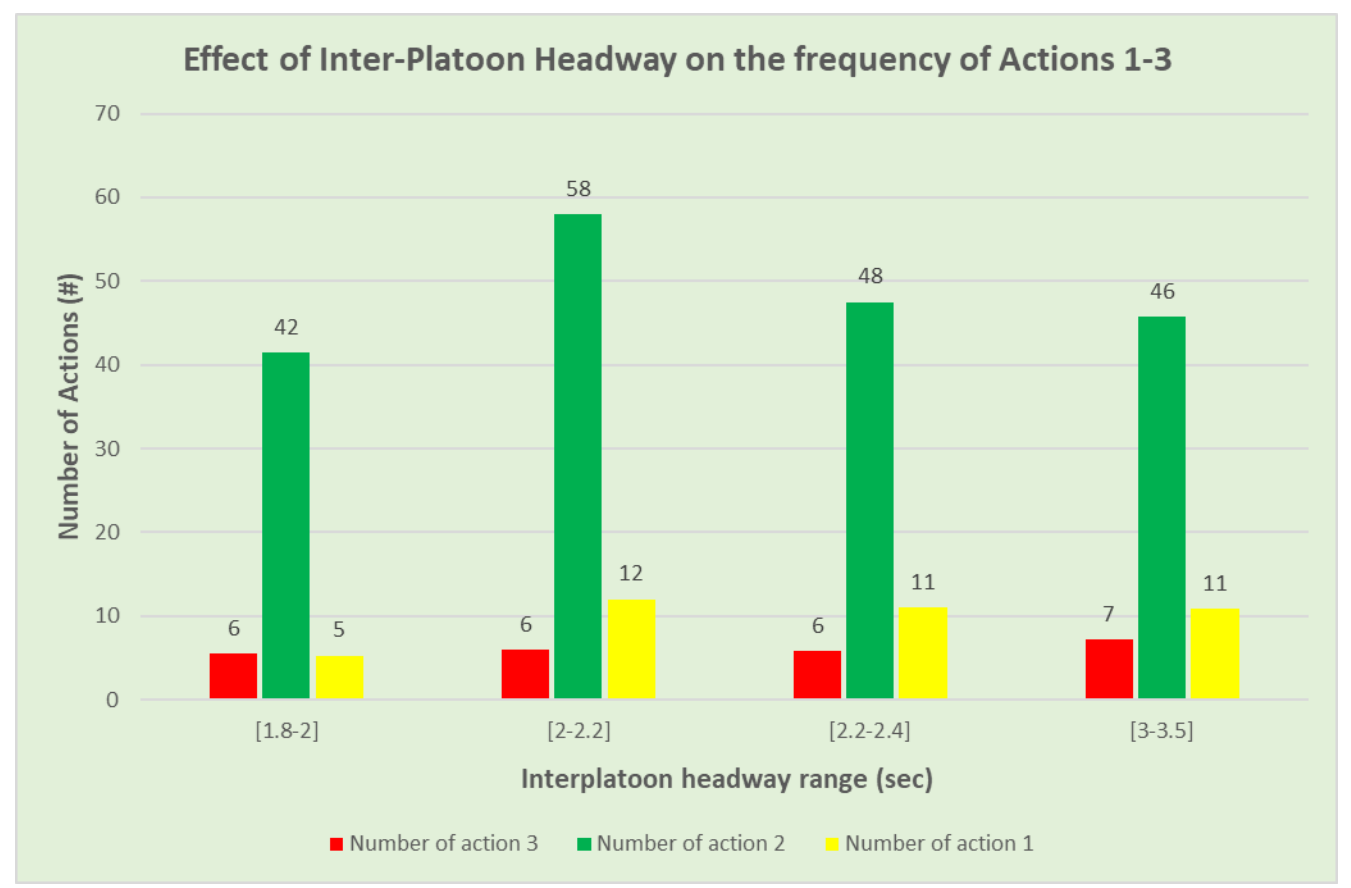

Figure 34 Effects of inter-platoon headway on number of actions 3 in a five-minute analysis 


\section{Approach 1. Platoon lane demand constant and rising onramp demand}

This approach is examining the effect of rising onramp demand on the throughput of the merge area when the platoon lane demand is constant. The approach aims at understanding how the throughput of the merge area can be influenced by rising onramp demand. This increasing onramp demand is limited to the model operational thresholds. Table 29 displays the effect of rising onramp demand on the merge area throughput and frequency of action 3. As can be seen, higher onramp slightly increases the onramp demand while increasing the frequency of action 3.

\section{Table 29 Analysis of number of the relation of action 3 with rising onramp demand}

\begin{tabular}{|c|c|c|c|}
\hline \multicolumn{2}{|c|}{ Model Inputs } & \multicolumn{2}{c|}{ Model outputs } \\
\multicolumn{2}{|c|}{ Platoon length = [3-7] } & \multicolumn{2}{c|}{ Lane 1 demand = 2,180 vph } \\
\hline $\begin{array}{c}\text { Headway Range } \\
\text { on Onramp (s) }\end{array}$ & $\begin{array}{c}\text { Onramp } \\
\text { demand (vph) }\end{array}$ & $\begin{array}{c}\text { Median } \\
\text { number of } \\
\text { action 3 }\end{array}$ & $\begin{array}{c}\text { Merge } \\
\text { area } \\
\text { throughput } \\
\text { (veh/hr) }\end{array}$ \\
\hline$[2.5-3]$ & 1,310 & 84 & 8,800 \\
\hline$[3-3.5]$ & 1,108 & 105 & 8,712 \\
\hline$[3.5-4]$ & 960 & 62 & 8,520 \\
\hline
\end{tabular}

\section{Approach 2. Platoon lane demand and onramp demand rising}

This approach is examining the effect of rising onramp demand on the throughput of the merge area combined with the effect of rising platoon lane demand. The approach aims at understanding how the throughput of the merge area can be influenced by rising onramp demand and platoon lane demand simultaneously. This increasing onramp demand and the platoon lengths simulated are limited to the model operational thresholds. The results displayed in Table 30 and Figure 35-6 show that higher onramp demand and platoon lengths increase the throughput to a maximum $9,850 \frac{\mathrm{veh}}{\mathrm{hr}}$. Additionally, longer platoon lengths, raised the lane 3 throughput to more approximately 3,312vph. 
Table 30 The analysis of the simultaneous effect of longer platoons and higher onramp demand on the throughput of the merge area in 5 minutes

\begin{tabular}{|c|c|c|c|c|c|c|}
\hline \multirow{2}{*}{\begin{tabular}{l} 
Throughputs of \\
lane 3 with \\
varying onramp \\
demand (1 hr) \\
\multicolumn{1}{c}{ Average } \\
Platoon length \\
(\#)
\end{tabular}} & \multicolumn{2}{|c|}{$\begin{array}{l}\text { Onramp demand }=1220 \mathrm{vph} \\
\text { Headway } \operatorname{range}(\mathrm{s})=[2.8-3.1]\end{array}$} & \multicolumn{2}{|c|}{$\begin{array}{l}\text { Onramp demand }=1360 \mathrm{vph} \\
\text { Headway range }(\mathrm{s})=[2.5-2.8]\end{array}$} & \multicolumn{2}{|c|}{$\begin{array}{l}\text { Onramp demand }=1530 \mathrm{vph} \\
\text { headway range }(s)=[2.2-2.5]\end{array}$} \\
\hline & $\begin{array}{l}\text { Throughput } \\
\text { lane } 3 \text { (vph) }\end{array}$ & $\begin{array}{l}\text { Throughput } \\
\text { all lanes (vph) }\end{array}$ & $\begin{array}{l}\text { Throughput } \\
\text { lane } 3 \text { (vph) }\end{array}$ & $\begin{array}{l}\text { Throughput } \\
\text { all lanes } \\
\text { (vph) }\end{array}$ & $\begin{array}{l}\text { Throughput } \\
\text { lane } 3 \text { (vph) }\end{array}$ & $\begin{array}{l}\text { Throughput all } \\
\text { lanes (vph) }\end{array}$ \\
\hline$[2-5]$ & 2,616 & 8,112 & 2,650 & 8,184 & 2,500 & 8,304 \\
\hline [5 - 8] & 3,012 & 9,048 & 3,024 & 9,160 & 3,024 & 9,360 \\
\hline [8 - 11] & 3,264 & 9,564 & 3,252 & 9,600 & 3,312 & 9,828 \\
\hline
\end{tabular}




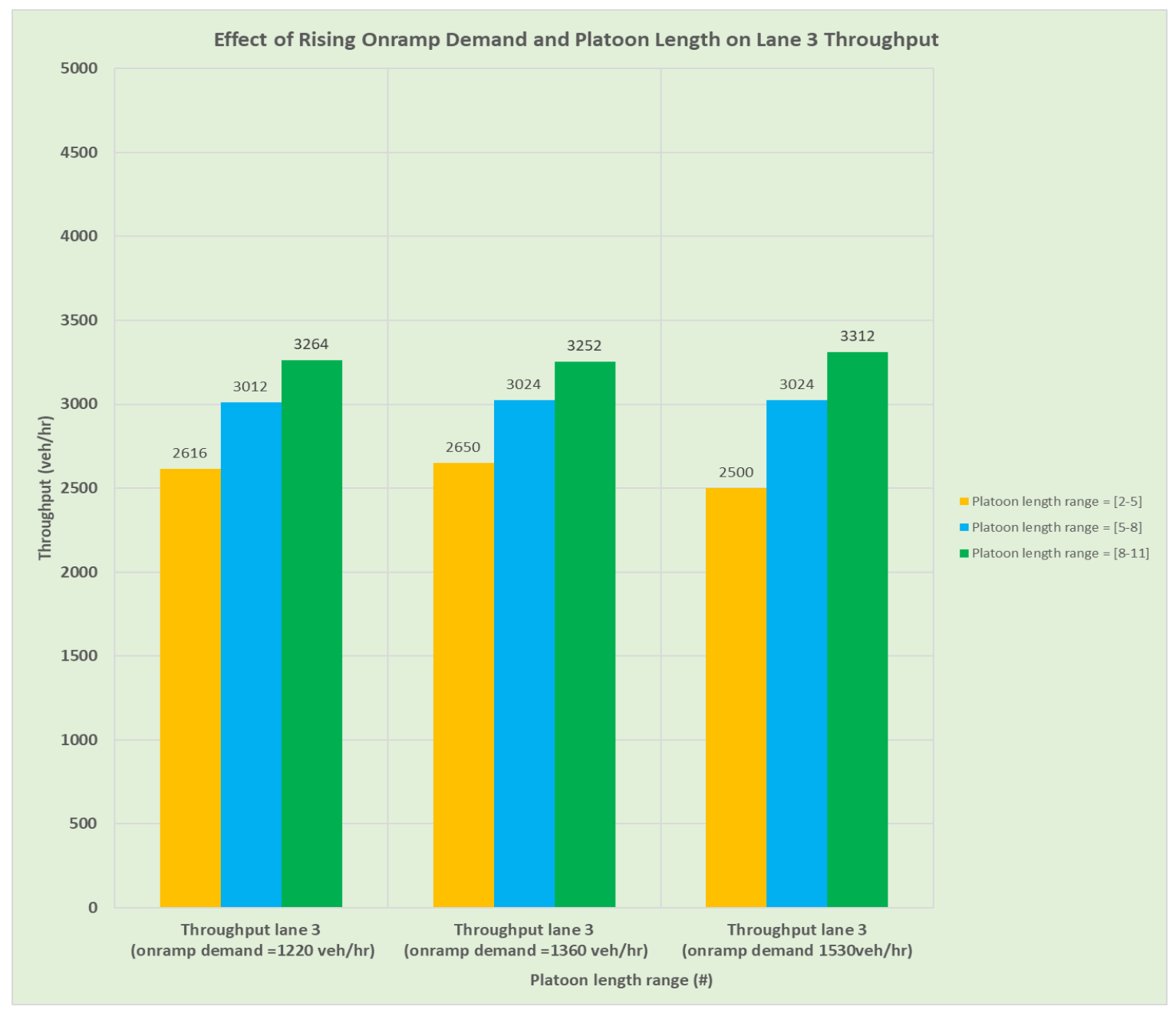

Figure 35 Analysis of the simultaneous effect of rising onramp and lane 1 demand on the lane 3 throughput 


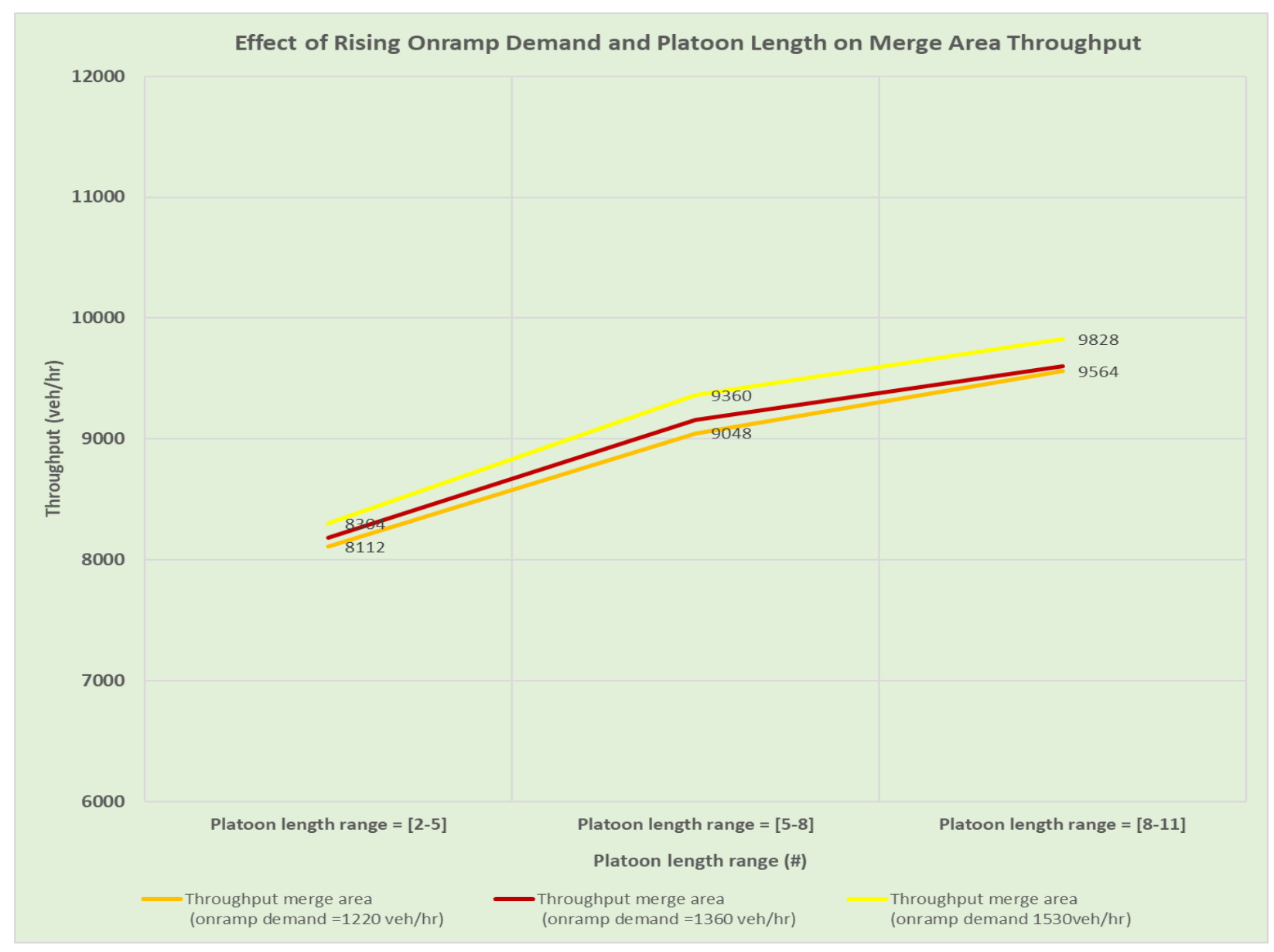

Figure 36 Analysis of the simultaneous effect of rising onramp and platoon lane demand on the merge area throughput 


\subsubsection{Result assessment}

The performance of the merge area was obtained by summarizing the results from the simulation tests. The rates below depict the highest throughputs obtained from the simulation results.

1. Lane 2 and 3 with maximum platoon length (average 9.5 vehicles per platoon and 0.7 seconds intra-platoon headway) is on average $3300 \frac{\mathrm{veh}}{\mathrm{hr}}$.

2. Maximum lane 1 throughput obtained $=3450 \frac{\mathrm{veh}}{\mathrm{hr}}$.

3. Maximum number of merging vehicles for a stable flow, added to a $2000 \frac{v e h}{h r}$ demand on lane 1 is $1400 \frac{v e h}{h r}$.

4. Maximum merge area throughput on all lanes for a stable flow is approximately $9300 \frac{\mathrm{veh}}{\mathrm{hr}}$.

\section{Recommended settings for the merge model:}

Table 31 below displays the recommended range of parameters which were grouped according the simulation tests for the operation of actions 1-3.

Table 32 displays the traffic performance with the recommended settings for the model, operating with actions 1-3. Table 33 displays the performance of the model with the recommended settings but with less onramp demand when operating with only action 1 .

The rates presented on Table 32 are not the optimum rates for the model input parameters. It is noteworthy to mention that obtaining the optimum result for each of these parameters may be unfeasible as many parameters have correlations, and also, the actual number of parameters to be optimized makes the problem mathematically inappropriate optimization problem. Therefore, the recommended settings below are obtained by evaluating the results of each simulation test and using an engineering judgement. 
Table 31 The model parameters as recommended based on the simulations of actions 1-3

\begin{tabular}{|c|c|}
\hline Parameter & Recommended result from this research \\
\hline Acceleration lane length $(\mathrm{m})$ & $250 \mathrm{~m}$ \\
\hline Length of danger zone (m) & $100 \mathrm{~m}$ \\
\hline Headway range lane 1 (sec) & [1.4-1.9] \\
\hline Headway range onramp (sec) & [2.2-2.9] \\
\hline Desired speeds on lane $1(\mathrm{~m} / \mathrm{s})$ & [35-38] \\
\hline Platoon length range (n) & [3-7] \\
\hline Inter-platoon headway (sec) & {$[2.2-2.7]$} \\
\hline Intra-platoon headway (sec) & {$[0.72]$} \\
\hline Platoon speeds $(\mathbf{m} / \mathbf{s})$ & [37-38] \\
\hline Minimum safe gap for merge & $50 \mathrm{~m}$ \\
\hline
\end{tabular}


The results of the experiments showed that the merging volumes can be increased to a maximum $1,400 \mathrm{vph}$ by the operation of action 2 and 3 compared to the sole operation of action 1 displayed on Table 32 and 33. The maximum throughput on lane 1 does not increase significantly with the operations of actions 2 and 3, but the lane change provides more gaps and subsequently more merging feasibility on lane 1. Furthermore, the joint operation of actions 2 and 3 reduce the frequency of action 1 and the percentage of vehicles merging through action 1, which is another positive outcome of those actions. Lower action 1 occurrence provides a more stable flow on lane 1. The merge area throughputs slightly increase with the operation of the actions 2 and 3. With the constraints of this model, the maximum lane 1 throughput rose from $2,000 \mathrm{vph}$ to $3,400 \mathrm{vph}$, while the merge area throughput increased from approximately 5,500vph to 9,400vph.

Table 32 Model performance of the recommended settings

\begin{tabular}{|c|l|l|l|r|r|r|l|l|}
\hline \multicolumn{7}{|c|}{ Model Inputs (lane 1 demand = 2180vph, onramp demand = 1400vph), platoon lengths (\#) =[3-7] } \\
\hline $\begin{array}{c}\text { Action } \\
\text { Operated }\end{array}$ & $\begin{array}{l}\text { Merged } \\
\text { volume } \\
\text { (vph) }\end{array}$ & $\begin{array}{l}\text { Frequency } \\
\text { of action 1 }\end{array}$ & $\begin{array}{l}\text { Frequency of } \\
\text { action }\end{array}$ & $\begin{array}{l}\text { Throughput } \\
\text { lane 1 (vph) }\end{array}$ & $\begin{array}{l}\text { Throughput } \\
\text { merge area } \\
\text { (vph) }\end{array}$ & $\begin{array}{l}\text { Percentage } \\
\text { merging } \\
\text { with } \\
\text { action 1 } \\
(\%)\end{array}$ & $\begin{array}{l}\text { Percentage } \\
\text { merging } \\
\text { with } \\
\text { action 2-3 } \\
(\%)\end{array}$ \\
\cline { 3 - 9 } & & $\mathbf{2}$ & $\mathbf{3}$ & & & & & \\
\hline $\mathbf{2}$ & 1,382 & 83 & 390 & - & 3190 & 9,275 & 6 & 28 \\
\hline $\mathbf{3}$ & 1,360 & 58 & 139 & 166 & 3,270 & 9,430 & 4 & 22 \\
\hline
\end{tabular}

Table 33 Traffic performance for the recommended settings with the operation of action 1

Model Inputs (lane 1 demand = 2180vph, onramp demand = 1200vph)
\begin{tabular}{|c|c|c|c|c|l|l|}
\hline $\begin{array}{l}\text { Action } \\
\text { Operated }\end{array}$ & $\begin{array}{l}\text { Merged } \\
\text { volume } \\
\text { (vph) }\end{array}$ & $\begin{array}{l}\text { Frequency } \\
\text { of action 1 }\end{array}$ & $\begin{array}{l}\text { Throughput } \\
\text { lane 1 (vph) }\end{array}$ & $\begin{array}{l}\text { Throughput } \\
\text { merge area } \\
\text { (vph) }\end{array}$ & $\begin{array}{l}\text { Percentage } \\
\text { merging with } \\
\text { action 1 (\%) }\end{array}$ & $\begin{array}{l}\text { Percentage } \\
\text { merging with } \\
\text { action 2-3 (\%) }\end{array}$ \\
\hline 1 & 1,146 & 92 & 3,342 & 9,116 & 8 & - \\
\hline
\end{tabular}




\section{DISCUSSION}

Traffic simulation models incorporate stochastic and deterministic input parameters. This characteristic of the models naturally leads to the performance measures being within a range. The model presented in this study also incorporated different stochastic and deterministic parameters to obtain the merge area performance of the proposed model. In the previous chapter the average of the range of each performance measure was presented. The recommended settings were selected according to the obtained results considering the conflict of gains between some outputs. In the next section the correlations and specifications of the outputs are discussed.

The simulation tests provided a platform the testing of different setups of the model parameters. The tests results were displayed in the previous section. In Table 34, the conflicting gains from an increase in the inter-platoon headway is displayed. Larger inter-platoon headways reduce the platoon lane throughout but increase the potential for lane change and subsequent merging of onramp vehicles.

\section{Table 34 Effect of inter-platoon length on the model outputs}

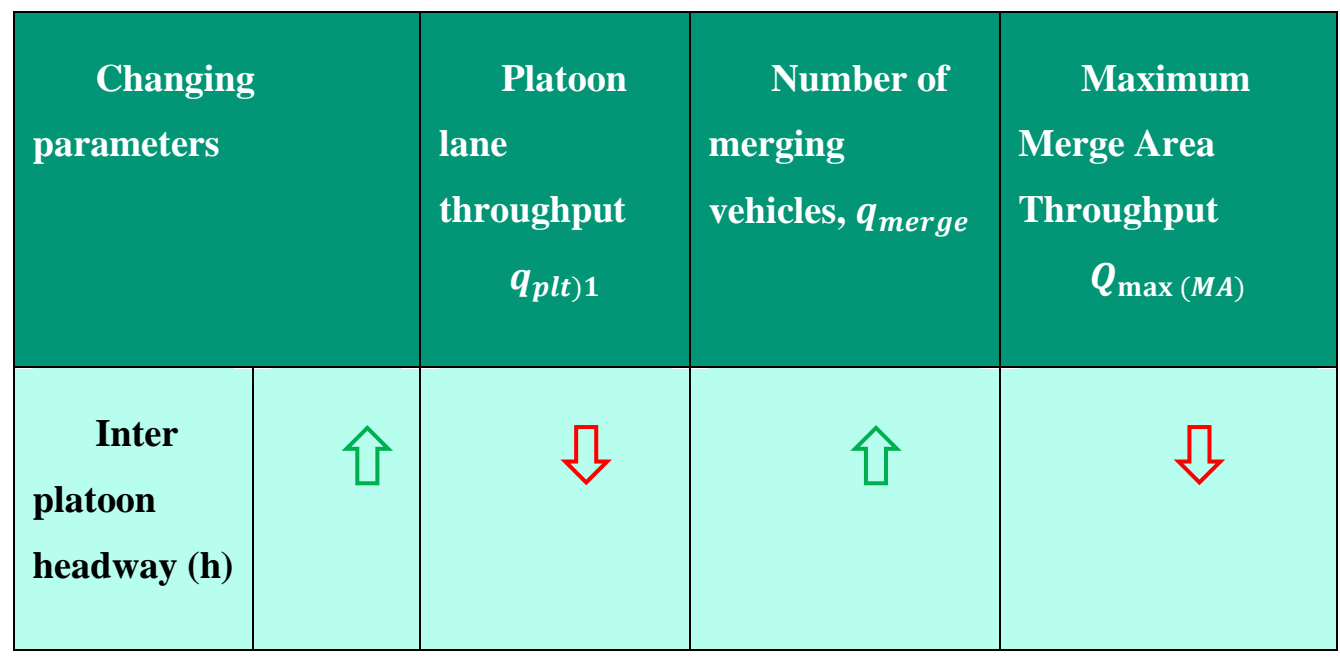

Table 35 displays the conflicting effects of inter-platoon headway and platoon length. Higher headways between platoons leads to vehicles in lane 1 to have more possibility to join a platoon. On the contrary, longer of platoons reduces the possibility of lane changes from lane 1 while increasing platoon lane throughput. 
Table 35 Correlation between parameters in the model

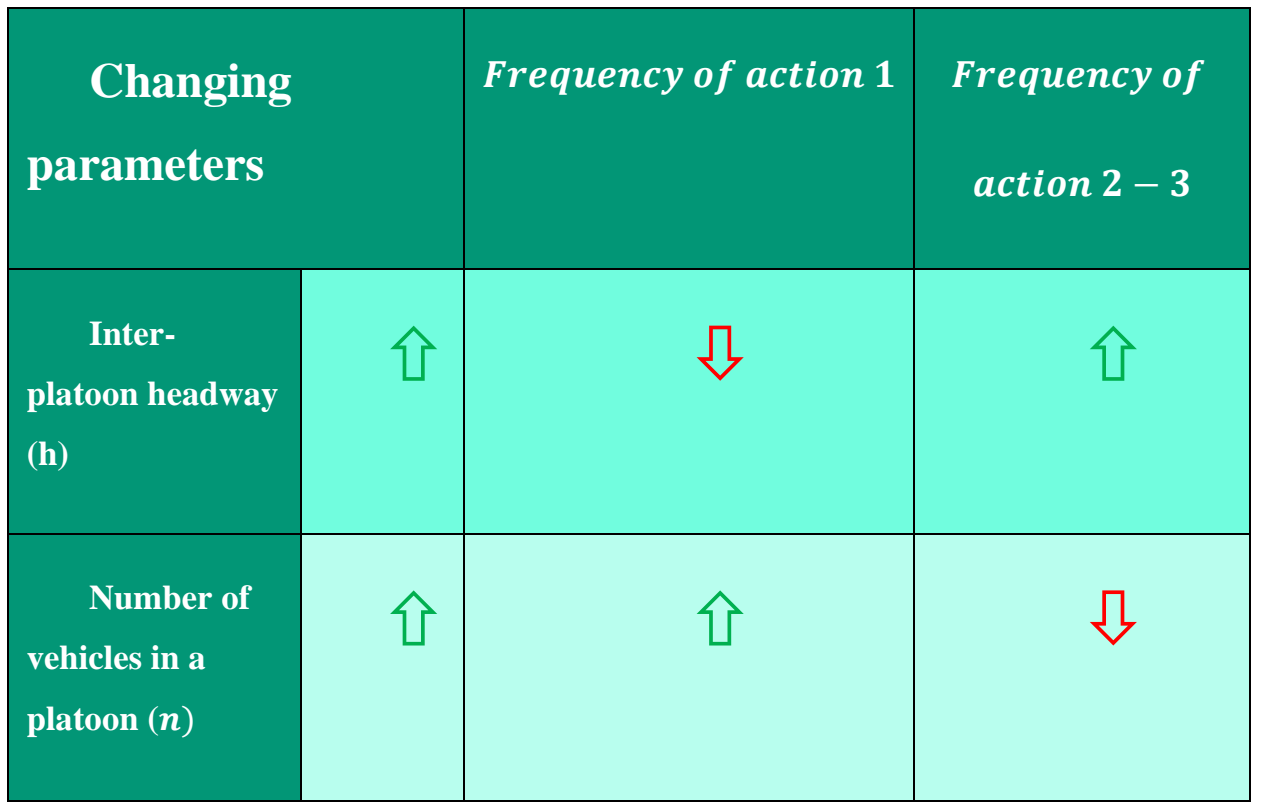

The maximum throughput on the platoon lanes are obtained when the platoon lane has the maximum length. On the other hand, the maximum platoon length will increase the platoon lane throughput while decreasing the lane change possibility from lane 1 . Therefore, there is a conflict between increasing platoon lane throughput and maximum merging throughput from the onramp. Though the lower lane change action has less impact on the reduction of lane 1 throughput compared to the higher throughput it yields on lane 2 and 3.

One of the parameters investigated in this research was the minimum safe gap required for merge on lane 1. This parameter has indirect relation with demand, merge throughput and frequency of actions on the mainlines. Longer defined safe gap, causes more actions on the mainlines, which is a countermeasure for the throughput of lane 1. Moreover, higher demands on lane 1 would create shorter gaps thus hampering the feasibility for merging vehicles. Therefore, in addition to the operation of action 2-3, there can be the possibility for the central controller to adjust the minimum safe gap based on the demand requirements of lane 1 and the onramp. As an example, it can be suggested that in higher demands, the minimum safe gap for merge can be reduced from $45 \mathrm{~m}$ to $40 \mathrm{~m}$.

The conditions presented for joining platoons in this research were limited to joining the front or rear end of a platoon. These conditions were assumed to prevent the deceleration of vehicles in a 
platoon. Advanced control and maneuvering systems which enable vehicles to join the middle of a platoon can form a more complex method for utilizing the additional capacity provided by lane 2 .

In this study the lane 1 demand was always higher than the onramp demand. This was due to the design of the merge model which required the lane 1 vehicles to provide the gaps for the onramp vehicles. Thus, the arrival rate of onramp vehicles had to be less than the arrival rate of the lane 1 vehicles. For modeling a merge area where in certain occasions, the merge throughput has priority over the mainline flow, the merge model is designed such that mainline vehicles arrive according to the gaps provided by the onramp merge traffic.

The intra-platoon headway is a key factor which determines the throughput of the platoon lane. In this study the range for this parameter was selected based on assumptions of the author and consultation with experts in this field ${ }^{4}$.

The results achieved in this study are presented as a guideline for future research in the opportunities of connectivity in the merge area and are not fixed determined results. Some model outputs such as the throughputs of the platoon and non-platoon lane are potential results, meaning that they may not necessarily be possible to create in real world traffic.

Certain outputs of the model required an engineering judgement for the recommendation of an adequate range. The length of the acceleration lane and the speed limits of the flows were parameters which were recommended through the evaluation of the results.

${ }^{4}$ (Dr. Taylor Lochrane, a fellow researcher in USDOT and an expert in the testing and operations of connected automated vehicles was contacted in this regard). 


\section{CONCLUSION AND FUTURE EXTENSIONS TO THE RESEARCH}

The model presented in this research aimed at testing the potential benefits of a rule-based control model for improving the performance of a merge area. The model was designed for a multilane highway comprised of platoon and non-platoon lane. Each onramp vehicle had a dedicated merging event for joining the lane 1 of the highway. The actions were limited to deceleration on lane 1 and lane change to the lanes 2 and 3. To incorporate the advent of ADAS in CAVs, the IDM+ car following model was enhanced with two additional controls.

Simulations were executed for understanding the effect of different model input parameters which were considered to have an effect on the performance of the merge area. The results displayed the change of merge behavior and performance in the merge area with the change of acceleration length, control parameters, and car following attributes. The modifications to the IDM+ car following model increased the throughput of the non-platoon lane from approximately 2,000 vph to $3400 \mathrm{vph}$. This increase enabled higher throughputs on lane 1 to accommodate the merging vehicles.

Major findings of this study are listed below:

- The minimum safe gap for merge is a key factor which determines the frequency of actions required for merging on the mainlines. The MOBIL lane change model applied in this research provides a minimum safe gap of 35 meters for the speeds of $30 \mathrm{~m} / \mathrm{s}$ which were used in the model. For additional safety concerns, the minimum safe gap was increased to $50 \mathrm{~m}$. This increment caused higher action requirements, leading to more deceleration on lane 1. The recommendation for the control in this study is $45 \mathrm{~m}$ which provides more safety than $35 \mathrm{~m}$ and leads to fewer actions compared to $50 \mathrm{~m}$. It must be noted that this parameter was tested for discrete lengths in this study and the results provided an insight on the parameter (the results obtained do not provide a fixed optimum length for the acceleration lane).

- The length of the acceleration lane is significant in reducing the need for deceleration for gap creation on lane 1. This geometric parameter becomes more significant if the merge area is only operating with action 1 . The acceleration lane length of $150 \mathrm{~m}$ proved to be inadequate for preventing actions on the mainlines. The acceleration lane of $200 \mathrm{~m}$ can be a reference for this parameter.

- Longer platoon lengths on lane 2 and 3 increase the throughput of those lanes, beside reducing the operational feasibility of vehicles to join the platoons. The results suggest that short platoons (e.g. 2 vehicles) are not efficient for the operation of the platoon lane, while 
long platoons (e.g. 10 vehicles) limit the feasibility of joining platoons. The recommendation of the author is platoons with [3-7] vehicles which yield approximately $3200 \mathrm{vph}$.

- Longer inter-platoon headways reduce the throughput of the platoon lane and did not significantly affect the frequency of vehicles joining platoons. Therefore, this parameter can be adjusted to the minimum safety requirements for platoons which in this study was 2.45 seconds.

This study provided an insight in the traffic of connected highways with CAVs. The findings of this study can be a guide for future designs of multilane highway merge areas. Additionally, the platoon lengths and car following proposed in this study can be assisting future research of highways with platoon and non-platoon lanes. The control model in this study can be further modified to conduct research of merge area with more advanced settings.

With the full automation of car following, the effect of the car following parameters simulated in this study can be further analyzed for the merge area throughput.

The results of this provide a framework for the performance of a multilane highway with platoon and non-platoon lanes. The model in this study can be applied for the analysis of the maximum throughputs in the merge area, but, does not provide any control on the system when the flow breaks down or is unstable. The rule-based control model introduced in this study is limited to certain actions to assist the merge and thus cannot be a benchmark for the performance of a controlled merge with the inputs of this study.

The car following model in the non-platoon lane of this study was constrained to certain thresholds which limited the potential inputs for the merge model. Furthermore, the settings for the platoon lanes were assumed based on the literature. Since there is no data on the specific features of platoons, the platoon lane parameters in this study cannot represent the actual future platoon lane traffic behavior.

In this study, every onramp vehicle merged through an action prior to the following onramp vehicle. This design limited the merging throughput of the model as two vehicles could not merge at the same time. Future extension of this work can include analyzing how merge area capacity can be improved by the entrance of onramp vehicles as platoons. Additionally, the model proposed in this study can be enhanced by the design of an algorithm which controls mainline vehicles gap creation according to a systematic planned entrance of the onramp entrance. The algorithm could maximize the mainline flow and onramp flow through an optimization problem. The model can be further enhanced 
with the harmonious gap creation on lane 1 for sequential entrance of merging vehicles in the form of platoons onto the ramp. In this condition the type of merging event can be formulated based on the number in the platoon of vehicles on the onramp.

Furthermore, with the full connection and automation of highways, a full platoon operating highway is envisioned. In that condition a platoon entering the onramp, will be joining a platoon on the mainlines. This would require algorithms for the merging of onramp platoons with mainline platoons.

For obtaining higher precision from a merge model, extensions to this study can include more accurate CACC car following models for all the lanes which would enable a microsimulation analysis of the vehicular movements. 


\section{References}

A. Omidvar, M. P. (2018). Optimizing Freeway Merge Operations under Conventional and Automated Vehicle Traffic Paper presented at the Transportation Research Board's 98th Annual Meeting, Washington D.C.

Ahmed, M. S., Hoque, M. A., Rios-Torres, J., \& Khattak, A. J. (2018). A Cooperative Freeway Merge Assistance System using Connected Vehicles. CoRR, abs/1805.00508.

Amoozadeh, M., Deng, H., Chuah, C.-N., Zhang, H. M., \& Ghosal, D. (2015). Platoon management with cooperative adaptive cruise control enabled by VANET. Vehicular Communications, 2(2), 110-123. doi: https://doi.org/10.1016/j.vehcom.2015.03.004

Bevly, D., Cao, X., Gordon, M., Ozbilgin, G., Kari, D., Nelson, B., . . Ozguner, U. (2016). Lane Change and Merge Maneuvers for Connected and Automated Vehicles: A Survey. IEEE Transactions on Intelligent Vehicles, 1(1), 105-120. doi: 10.1109/TIV.2015.2503342

Board, T. R., National Academies of Sciences, E., \& Medicine. (2017). Strategies to Advance Automated and Connected Vehicles. Washington, DC: The National Academies Press.

Clements, L. M., \& Kockelman, K. M. (2017). Economic Effects of Automated Vehicles. Transportation Research Record, 2606(1), 106-114. doi: 10.3141/2606-14

Dong, C., Dolan, J. M., \& Litkouhi, B. (2017). Interactive ramp merging planning in autonomous driving: Multi-merging leading PGM (MML-PGM). 2017 IEEE 20th International Conference on Intelligent Transportation Systems (ITSC), 1-6.

Fagnant, D. J., \& Kockelman, K. (2015). Preparing a nation for autonomous vehicles: opportunities, barriers and policy recommendations. Transportation Research Part A: Policy and Practice, 77, 167-181. doi: https://doi.org/10.1016/j.tra.2015.04.003

Fernandes, P., \& Nunes, U. (2015). Multiplatooning Leaders Positioning and Cooperative Behavior Algorithms of Communicant Automated Vehicles for High Traffic Capacity. IEEE Transactions on Intelligent Transportation Systems, 16(3), 1172-1187. doi: 10.1109/TITS.2014.2352858

Gereon Meyer, S. B. (2014). Road Vehicle Automation.

Handbook, FHWA HANDBOOK.

Haneen Farah, S. M. J. G. E., Tom Alkim, Bart van Arem. (2018). Road Vehicle Automation 4: Springer.

Hussain, Syed A.; Shahian Jahromi, Babak; Cetin, Sabri. 2020. Cooperative Highway Lane Merge of Connected Vehicles Using Nonlinear Model Predictive Optimal Controller. Vehicles 2, no. 2: 249-266.

Jones, S. (2013). Cooperative Adaptive Cruise Control: Human Factors Analysis. 
Kamali, M., Dennis, L. A., McAree, O., Fisher, M., \& Veres, S. M. (2017). Formal verification of autonomous vehicle platooning. Science of Computer Programming, 148, 88-106. doi: https://doi.org/10.1016/j.scico.2017.05.006

Karbalaieali, S., Osman, O. A., \& Ishak, S. (2018). Evaluation of a Novel Model for Cooperative Merging of Connected Automated Vehicles onto Automated Highways. Paper presented at the Transportation Research Board 97th Annual Meeting, Washington DC, United States.

Kesting, A., Treiber, M., \& Helbing, D. (2007). General Lane-Changing Model MOBIL for CarFollowing Models. Transportation Research Record, 1999(1), 86-94.

Larson, J., Munson, T., \& Sokolov, V. (2016). Coordinated Platoon Routing in a Metropolitan Network 2016 Proceedings of the Seventh SIAM Workshop on Combinatorial Scientific Computing (pp. 73-82).

Lioris, J., Pedarsani, R., Tascikaraoglu, F. Y., \& Varaiya, P. (2017). Platoons of connected vehicles can double throughput in urban roads. Transportation Research Part C: Emerging Technologies, 77, 292-305. doi: https://doi.org/10.1016/j.trc.2017.01.023

Marinescu, D., Čurn, J., Bouroche, M., \& Cahill, V. (2012, 16-19 Sept. 2012). On-ramp traffic merging using cooperative intelligent vehicles: A slot-based approach. Paper presented at the 2012 15th International IEEE Conference on Intelligent Transportation Systems.

Michaud, F., Lepage, P., Frenette, P., Letourneau, D., \& Gaubert, N. (2006). Coordinated Maneuvering of Automated Vehicles in Platoons. IEEE Transactions on Intelligent Transportation Systems, 7(4), 437-447. doi: 10.1109/TITS.2006.883939

Milanés, V., Shladover, S. E., Spring, J., Nowakowski, C., Kawazoe, H., \& Nakamura, M. (2014). Cooperative Adaptive Cruise Control in Real Traffic Situations. IEEE Transactions on Intelligent Transportation Systems, 15(1), 296-305. doi: 10.1109/TITS.2013.2278494

Na Chen, W., AlkimB, van Arem. (2018). A Flexible Strategy for Efficient Merging Maneuvers of Connected Automated Vehicles. Paper presented at the 18th COTA International Conference of Transportation Professionals.

Nowakowski, C., O'Connell, J., Shladover, S., \& Cody, D. (2010). Cooperative Adaptive Cruise Control: Driver Acceptance of Following Gap Settings Less than One Second (Vol. 3).

Ntousakis, I. A., Nikolos, I. K., \& Papageorgiou, M. (2016). Optimal vehicle trajectory planning in the context of cooperative merging on highways. Transportation Research Part C: Emerging Technologies, 71, 464-488. doi: https://doi.org/10.1016/j.trc.2016.08.007

Öncü, S., Ploeg, J., Wouw, N. v. d., \& Nijmeijer, H. (2014). Cooperative Adaptive Cruise Control: Network-Aware Analysis of String Stability. IEEE Transactions on Intelligent Transportation Systems, 15(4), 1527-1537. doi: 10.1109/TITS.2014.2302816

Park, H., Bhamidipati, C. S., \& Smith, B. L. (2011). Development and Evaluation of Enhanced IntelliDrive-Enabled Lane Changing Advisory Algorithm to Address Freeway Merge Conflict. Transportation Research Record, 2243(1), 146-157. doi: 10.3141/2243-17 
Rahman, M., Chowdhury, M., Dey, K., Islam, M. R., \& Khan, T. (2017). Evaluation of Driver CarFollowing Behavior Models for Cooperative Adaptive Cruise Control Systems.

Transportation Research Record: Journal of the Transportation Research Board, 2622, 8495. doi: 10.3141/2622-08

Rathgeber, C., Winkler, F., Kang, X., \& Müller, S. (2015). Optimal Trajectories for Highly Automated Driving.

Rios-Torres, J., \& Malikopoulos, A. A. (2017). Automated and Cooperative Vehicle Merging at Highway On-Ramps. IEEE Transactions on Intelligent Transportation Systems, 18(4), 780789. doi: 10.1109/TITS.2016.2587582

Scarinci, R., \& Heydecker, B. (2014). Control Concepts for Facilitating Motorway On-ramp Merging Using Intelligent Vehicles. Transport Reviews, 34(6), 775-797. doi:

10.1080/01441647.2014.983210

Schakel, W. J., Knoop, V. L., \& van Arem, B. (2012). Integrated Lane Change Model with Relaxation and Synchronization. Transportation Research Record, 2316(1), 47-57. doi: 10.3141/2316-06

Shen, M., Hu, H., Sun, B., \& Deng, W. (2018). Heuristics Based Cooperative Planning for Highway On-Ramp Merge. 201821 st International Conference on Intelligent Transportation Systems (ITSC), 1266-1272.

Subraveti, H. H. S. N., Knoop, V. L., \& Arem, B. v. (2018). Rule based control for merges: Assessment and case study. 201821 st International Conference on Intelligent Transportation Systems (ITSC), 3006-3013.

Talebpour, A., \& Mahmassani, H. S. (2016). Influence of connected and autonomous vehicles on traffic flow stability and throughput. Transportation Research Part C: Emerging Technologies, 71, 143-163. doi: https://doi.org/10.1016/j.trc.2016.07.007

Tiernan, T. A., Richardson, N., Azeredo, P., Najm, W. G., \& Lochrane, T. (2017). Test and Evaluation of Vehicle Platooning Proof-Of-Concept Based on Cooperative Adaptive Cruise Control.

Treiber, Hennecke, \& Helbing. (2000). Congested traffic states in empirical observations and microscopic simulations. Physical review. E, Statistical physics, plasmas, fluids, and related interdisciplinary topics, 622 Pt A, 1805-1824.

Tsugawa, S., Kato, S., Tokuda, K., Matsui, T., \& Fujii, H. (2001, 25-29 Aug. 2001). A cooperative driving system with automated vehicles and inter-vehicle communications in Demo 2000. Paper presented at the ITSC 2001. 2001 IEEE Intelligent Transportation Systems. Proceedings (Cat. No.01TH8585).

Varaiya, P. (1993). Smart cars on smart roads: problems of control. IEEE Transactions on Automatic Control, 38(2), 195-207. doi: 10.1109/9.250509

Xie, Y., Zhang, H., Gartner, N. H., \& Arsava, T. (2017). Collaborative merging strategy for freeway ramp operations in a connected and autonomous vehicles environment. Journal of Intelligent Transportation Systems, 21(2), 136-147. doi: 10.1080/15472450.2016.1248288 
Xiangwang Hu, Jian Sun. (2019). Trajectory optimization of connected and autonomous vehicles at a multilane freeway merging area. Transportation Research Part C: Emerging Technologies, Volume 101, Pages 111-125

Zhao, L., \& Sun, J. (2013). Simulation Framework for Vehicle Platooning and Car-following Behaviors Under Connected-vehicle Environment. Procedia - Social and Behavioral Sciences, 96, 914-924. doi: https://doi.org/10.1016/j.sbspro.2013.08.105

Zhong, Z., Lee, J., \& Zhao, L. (2017). Multiobjective Optimization Framework for Cooperative Adaptive Cruise Control Vehicles in the Automated Vehicle Platooning Environment. Transportation Research Record, 2625(1), 32-42. doi: 10.3141/2625-04 


\section{Appendix}

The code structure for the operation of the model is attached below.

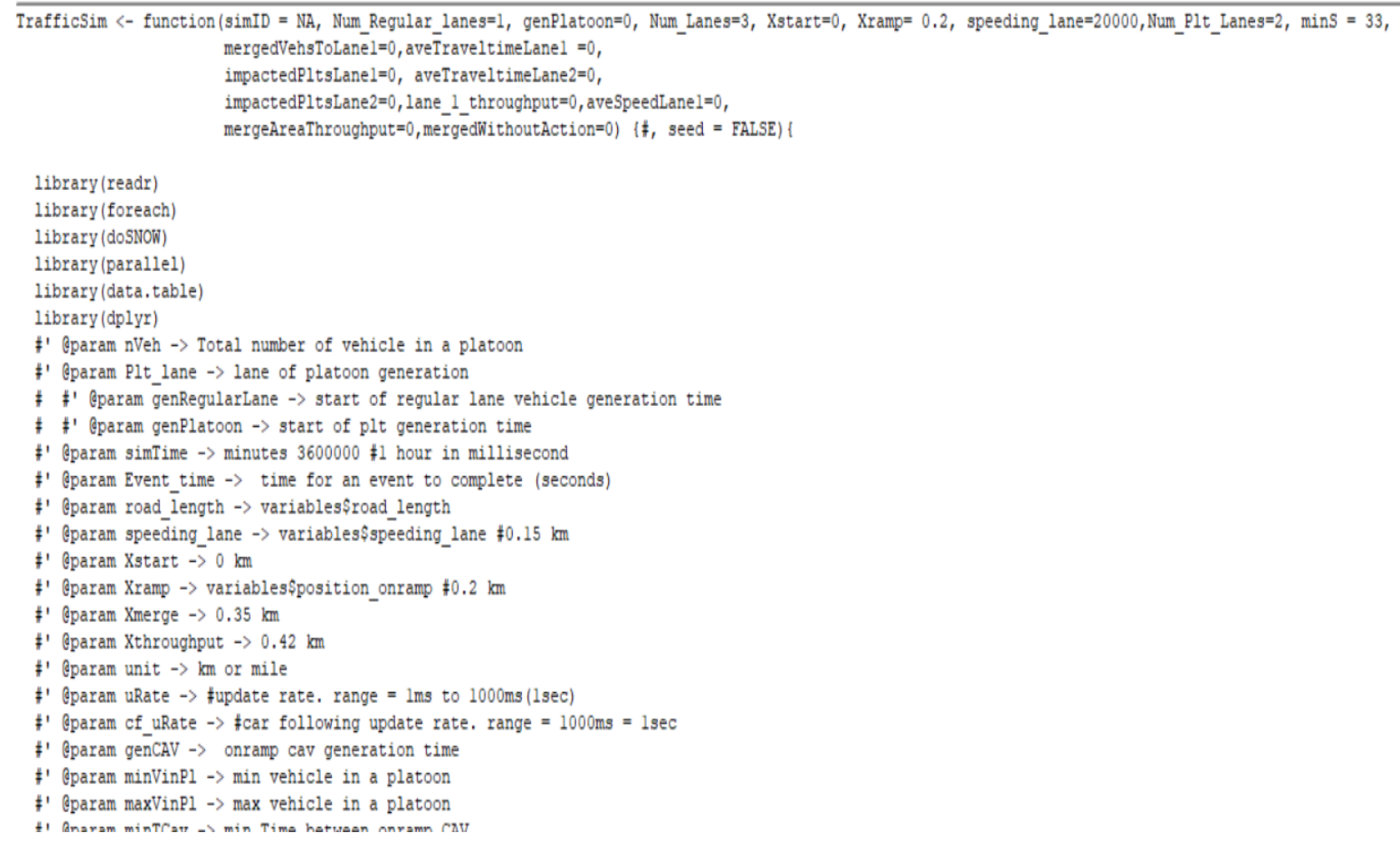


\#ithin the simulation time (continue next line)

\#for every event

\#From the start of event, update the feature vector dataframe and simulation dataframe

\#the feature vector dataframe is dynamic and updates every milisecond, the simulation dataframe which is recording the events and is completed after every even

\# dataframes in generation of vehicles

pltLaneDynNames <- sprintf ("plt Lane so2d Dyn", 1:Num P1t Lanes)

pltLaneReportNames <- sprintf ("plt_Lane_ö02d_Report", 1:Nü_P1t_Lanes

regLaneDynNames <- sprintf ("reg_Lane_orod_Dyn", 1:Num_Regular_lanes)

regLaneReportNames <- sprintf ("reg_Lane_so2d_Report", 1:Num_Regular_lanes)

rampDynames <- sprintf ("ramp_so2d_Dyn", 1:rampLanes)

rampReportNames <- sprintf ("ramp_ so-2d_Report", 1 : rampLanes)

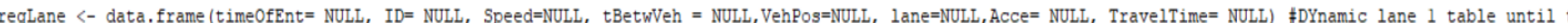
pltLane <- data.frame (ID= NULL, nVehPl=NULL, Net_Headway_Pla=NULL, bufferZone=NULL, pltLength=NULL, Speed = NULL, Acce= NULL, lane=NULL, time0fEnt=NULL, pltPos=NULL, ramp <- data.frame (ID=NULL, tBetwCav=NULL, speed=NULL, timeOfEnt= NULL, VehPos=NULL, TravelTime= NULL) \#\# dynamic dataframe

assign ('finalReport', data.frame ( $\mathrm{t} m \mathrm{~s}=0, \mathrm{ID}=0$, vehID $=0$, speed $=0$, type $=0$, Traveltime $=0)$ ) $\#$, timeofEnt $=0$ ) $¥$ Report table of platoon generations

assign ('next_plt_gen_time', data.frame (laneNumber $=1$ :Num_Plt_Lanes, plt_gen_time $=0)$ ) \#Lookup table for checking time of new pltoon generation

assign ('finalReport', data. frame ( $\mathrm{ms}=0$, vehID $=0$, speed $=0$, type $=1$, Traveltime $=0$ ) ) $\#$, time 0 fEnt $=0$ ) $\#$ Report table of platoon generations

assign ('next_veh_gen_time', data.frame (laneNumber $=1$ :Num_Regular_lanes, veh_gen_time $=0)$ ) \#Lookup table for checking time of new reg lane generation

assign ('finalReport', data.frame (t_ms $=0$, vehID $=0$, speed $=0$, type $=2$, Traveltime $=0)$ ) $\$$, time 0 fEnt $=0$ ) $¥$ Report table of platoon generations

assign ('next_cav gen time', data.frame (laneNumber = 1 :rampLanes, cav gen time = genCAV)) \#Lookup table for checking time of new CAV generation

mainline Densities <- data.frame $(t=N A)$ \#Dataframe of densities

pltLaneDenT <- sprintf("plt_Lane_so2d", 1:Num_P1t_Lanes)

regLaneDenT <- sprintf("reg Lane so2d", 1:Num Regular lanes)

mainline Densities [c(regLaneDenT, pltLaneDenT) $\overline{\text { ] }}<-$ NA

mergeEvents <- data.frame (actionType=NULL, nlanechange=NULL, Decetime=NULL, cost=NULL

newPlatoon <- T \#\#\# application?

\#\#\# Define 3 simulation time. One for the update of feature vectors, one for the recording events and one for the end of simulation

$t<-0$

\#\# $t$ is update time for feature vector of all vehicles

Event_time <- $0 \quad \# \#$ event time which records the time of each event and restarts for each event

stopsim $<-T \quad \# \#$ time to stop simulation

car_following_time <- 0 \#\# time to update car following features

\#rmpVehPos $<-$ Xramp

VehPos <- Xstart

pltPos <- Xstart

\#uRate <- 1000 \#update rate. range $=1 \mathrm{~ms}$ to $1000 \mathrm{~ms}$ (1sec)

pltId $<-0 \quad$ \#Initial platoon number

cavId <- $0 \quad$ \#Initial cav number

Regular_LaneID $<-0$ \#Initial lane 1 vehicle number

genPlatoon <- 0 \#time for the start of platoon generation

genRegularLane <- 0 \#time for the start of lane 1 vehicle generation

minspacing <- $1000 \quad \# 10 \mathrm{~m}$ or $1000 \mathrm{~mm}$ for merging

deceTime $<-0$

gap required <- 5200 \#\# from the figure 14.2 in book

nextMaxGap $<-0$

gapID $<-0$

nLanechange $<-0$

deceCost $<-0$

targetVehID $=\mathrm{NA}$

posDif $=0$

spdDif $=0$

gapsInRange $<-\mathrm{NA}$

$\#$ minVinP1 $=2$

$\# \max$ inP1 $=2$

$\$ \operatorname{minTCav}=(3600 / \mathrm{nCav})-1$

$\#$ maxTCav $=(3600 / \mathrm{nCav})+1$

\#Note: Distance is in $\mathrm{cm}$ level and time is millisecond level. The reason is for not dealing with decimal numbers. 
if (! is.na(nextMaxGap) \& nextMaxGap $>=$ gap_required \& maxGapMerge $<$ gap_required \& mergingCAV\$VehPos $<$ speeding_lane+ 20000-7000 \& maxGapMerge $>0)$ \& print (paste ("nextMaxGap=", nextMaxGap, "MaxGap =", maxGapMerge))

$\$ \#$ function for merge vehicle actions to join the gap

Adaption_to_gap <- AdaptionToGap (ramp_01_Dyn = ramp_01_Dyn, \#\# function for merging

ramp_01_Report $=$ ramp_01_Report

mergingCAV=mergingCAV, nextMaxGap=nextMaxGap,

\#mergingCAVGroup=mergingCAVGroup,

\#speeding lane=speeding lane,

reg_Lane_01_Dyn=reg_Lane_01_Dyn, gapID=gapID, speeding_lane=speeding_lane,

\#NumInGroup=NumInGroup,

\#targetVehID=targetVehID)

frontCAV = frontCAV, posDif=posDif, spdDif=spdDif $) \neq$, end_accel_lane=end_accel_lane,

ramp_01_Report <- Adaption_to_gap\$ramp_01_Report

reg_Lane_01_Dyn<- Adaption_to_gap\$reg_Lane_01_Dyn

ramp_01_Dyn <- Adaption_to_gapsramp_01_Dyn

posDif <- Adaption to gap\$posDif

spdDif <- Adaption_to_gap\$spdDif

gapID <- Adaption to gap\$gapID

print (paste ("next $\max$ gap =", nextMaxGap, "max gap merge is ", maxGapMerge))

lelsef

print (maxGapMerge)

if (maxGapMerge $>=$ gap_required) $\{\# \#$ (is required gap available)

leaderID <- (reg_Lane_01_Dyn\$ID [reg_Lane_01_Dyn\$gap $=$ maxGapMerge $) \# \&$ reg_Lane_01_Dyn\$VehPos $>=20000 \&$ reg_Lane_01_Dyn\$VehPos $\langle=25000)], 0)$

followerID <- leaderID + 1

\#mergingCAVGroup\$ID <- (leaderID + 1)+100 : (leaderID + 1) +(NumInGroup -1$)+100$ \#\#ID of merged CAVs i the reg lane

targetVehID $<-$ followerID

ramp_merge <- Onramp_merge (ramp_01_Dyn = ramp_01_Dyn,

ramp_01_Report $=$ ramp_01_Report,

\#\# function for merging

merging $\mathrm{CAV}=$ mergingCAV,

\#mergingCAVGroup=mergingCAVGroup,

speeding_lane=speeding_lane,

reg_Lane_01_Dyn=reg_Lane_01_Dyn,

NumInGroup=NumInGroup,

followerID=followerID

targetVehID=targetVehID)

leader ID=leaderID)

ramp_01_Report <- ramp_merge $\$$ ramp_01_Report

reg_Lané_01_Dyn<- ramp_mergełreg_Lané_01 Dyn

ramp_01_Dyn <- ramp_merge\$ramp_01_Dyn

gap_Projection <- gapProjection (gap_required=gap_required, ramp_01_Dyn = ramp_01_Dyn,

ramp_01_Report = ramp_01_Report,

merging $\overline{C A V}=$ merging $C A V, \max$ GapMerge=maxGapMerge,

\#mergingCAVGroup=mergingCAVGroup,

\#speeding_lane=speeding_lane,

reg_Lane 01 Dyn=reg_Lane 01 Dyn, spdDif=spdDif, posDif=posDif,

\#NumInGroup=NumInGroup,

\#followerID=followerID,

front $C A V=$ front $C A V$ )

\#leaderID=leaderID, reglanedensities=reglanedensities,

\#targetVehID = targetVehID, maxNumMerge=maxNumMerge)

ramp_01_Report <- gap_Projection\$ramp_01_Report

reg_Lane_01_Dyn<- gap_Projection\$reg_Lane_01_Dyn

ramp_01_Dyn ${ }_{-}<-$gap_Projection\$ramp_01_Dyn

nextMaxGap <- gap Projection\$nextMaxGap

posDif <- gap_Projection\$posDif

gapID <- gap_Projection\$gapID 
Yelse

if (ramp_01_Dyn\$VehPos [which (ramp_01_Dyn\$ID=efrontCAV)] $>=($ speeding_lane+20000) -7000$) 1$

mergeAction <- mergeTypel (gap required=gap required, ramp 01 Dyn = ramp 01 Dyn,

\#\# function for cooperative merging

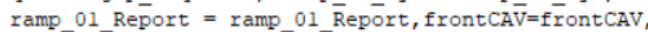

merging $\bar{C} A V=$ mergingCAV, $\max$ GapMerge=maxGapMerge,

\#mergingCAVGroup=mergingCAVGroup,

speeding_lane=speeding_lane,

reg_Lane_01_Dyn=reg_Lane_01_Dyn,

NumInGroup= $=\bar{N}$ umInGroup, deceTime=deceTime,

followerID=followerID, nLanechange=nLanechange, deceCost = deceCost,

plt_Lane_01_Dyn = plt_Lane_01_Dyn,

plt_Lane_02_Dyn $=$ plt_Lane_02_Dyn, mergeEvents=mergeEvents,

reglanedensities=reglanedensities,

pltLaneDensities=pltLaneDensities,

targetVehID = targetVehID, maxNumMerge=maxNumMerge)

\#\#\# A.1 cost function parameters and calculations to come here

\# \# assign(sprintf("plt Lane \&02d Dyn", i), mergeAction\$plt LaneTab)

\# \# assign (sprintf("plt_Lane_so2d_Report", i), mergeAction\$plt_LaneTab2)

plt_Lane_01_Dyn = mergeAction\$plt_Lane_01_Dyn

plt_Lane_02_Dyn $=$ mergeAction\$plt_Lane_02_Dyn

ramp_01_Report <- mergeAction\$ramp_01_Report

reg_Lané 01 Dyn<- mergeAction\$reg_Lané_01_Dyn

ramp_01_Dyn <- mergeAction\$ramp_01_Dyn

dece Timé <- mergeAction\$deceTimé

targetVehID <- mergeAction\$targetVehID

deceCost <- mergeAction\$deceCost

\#nLanechange <-mergeAction\$nLanechange

mergeEvents <- mergeAction\$mergeEvents

if $\left(t==\left(\right.\right.$ simTime $\left.\left.^{\star} 60000\right)\right)\{$ \#Convert simulation time minute to millisecond. Stop simulation if exceeded the defined time stopSim $=\mathrm{F}$ \}

1 \# end of while

a <- mean(reg Lane 01 Report\$TravelTime)

$\mathrm{b}<-$ mean (plt_Lane_01_Report\$TravelTime)

c <- mean (plt Lane 02 Report\$TravelTime)

meanTravelTimeAlltanes $<-(a+b+c) / 3$

mergedWithoutAction <- (nrow (ramp_01_Report [(ramp_01_Report\$̣type ==2), ])/nrow (ramp_01_Report)) * 100

mergedVehsToLane1 <- nrow (ramp_01_Report)

impactedP1tsLane1 <- nrow (plt__ane_01_Report [(plt_Lane_01_Report\$type ==5), ]) + nrow (plt_Lane_01_Dyn [(plt_Lane_01_Dyn\$type ==5), ])

impactedP1tsLane2 <- nrow (plt_Lane_02_Report [(plt_Lane_02_Report\$type ==5), ]) + nrow (plt_Lane_02_Dyn[( plt_Lane_02_Dyn\$type ==5), ])

aveTraveltimeLane1 <- mean(reg_Lane_0 $\overline{1}_{-}$Report\$TravelTime)

aveTraveltimeLane $2<-$ mean (plt Lane 01 Report\$TravelTime)

aveSpeedLane $<-($ road_length $\bar{*} 100 \overline{0}) \bar{T}$ (aveTraveltimeLanel/1000)

mergeAreaThroughput <- nrow (reg_Lane_01_Report) + sum(p1t_Lane_01_Report\$nVehP1) + sum (plt_Lane_02_Report\$nVehP1)

lane_1_throughput <- nrow (reg_Lane_01_Report) 
results <- data.frame (id= simID, Throughput_all_lanes = mergeAreaThroughput, Merged Vehicles $=$ mergedVe $\bar{s}$ ToLanel,

platoons impacted lane 2 = impactedPltsLane2,

platoons_impacted_lane_1 = impactedPltsLanel,

Overall_Äverage_travel_time = meanTravelTimeAllLanes,

TT_Lanel=aveTravel timeLane1,

TT_Lane2=aveTravel timeLane2, aveSpeedLane1=aveSpeedLane1,

lane_1_throughput=1ane_1_throughput, Merging_With_NoAction= mergedWithoutAction)

View (as.data.frame (results))

\#write_csv(results, "results.csv", append = TRUE, col_names = TRUE)

return (trajectories)

\#\# temporary end of mainline traffic sim function 


\title{
Ehsan Beheshtitabar
}

\section{Education}

\author{
Old Dominion University, Norfolk, Virginia \\ April 2020 \\ Ph.D. in Transportation Engineering \\ Linkoping University, Linkoping, Sweden \\ Dec. 2013 \\ Master's Degree Transportation Systems Engineering \\ Thesis: Calibration of the 2+1 Link Model in the Rural Road Traffic Simulator, RUTSIM \\ University of Applied Science Technikum Vienna, Vienna, Austria, \\ July 2012 \\ Master's Degree in Intelligent Transport Systems Engineering
}

Yazd University, Yazd, Iran

Dec. 2010

Bachelor of Science in Civil Engineering

\section{Select Publications and Presentations}

1. E. Beheshtitabar, S. Aguilar, Z. Svaty, D. Konig and C. Rydergren. "Route Choice Modelling for Bicycle Trips". International Journal for Traffic and Transport Engineering, Volume 4(2), 2014 (IJTTE).

2. E. Beheshtitabar and M. Cetin. "Impacts of Downstream Observability on the Traffic Performance within a Tunnel". 2019 International Conference on Transportation \& Development (ICTD 2019).

3. R. Nezafat, E. Beheshtitabar, M.Cetin, E.Williams and G.List. "Modeling and Evaluating Traffic Flow at Sag Curves When Imposing Variable Speed Limits on Connected Vehicles". Transportation Research Record, 2018.

4. E. Beheshtitabar and E. Alipour. "A Rule Based Model for Merging onto Connected Automated Highways". 2020 International Conference on Transportation \& Development (ICTD 2020).

5. B. Ghadirifaraz, M.Fotoohi, M.Vaziri and E. Beheshtitabar. "A Pedestrian Crash Prediction Model for Urban Signalized Intersections Case Study: The City of Isfahan". 98th Annual Meeting of Transportation Research Board, 2019.

6. M. Arabi, E. Beheshtitabar, B.Ghadirfaraz and B. Forjanizadeh. "Optimum Locations for Intercity Buses Terminals with the AHP Approach - Case Study of the City of Esfahan". 8th International Conference on Transport, Structure and Environment (London, 2015).

7. E. Beheshtitabar, R. Nezafat, M.Cetin, E.Williams and G.List. "The Influence of Look Ahead Distance on Reducing Average Travel Times Within A Sag Curve”. SDITE 2017 Annual Meeting. Columbia. South Carolina. 